\title{
$n$-ABELIAN AND $n$-EXACT CATEGORIES
}

\author{
GUSTAVO JASSO
}

\begin{abstract}
We introduce $n$-abelian and $n$-exact categories, these are analogs of abelian and exact categories from the point of view of higher homological algebra. We show that $n$-cluster-tilting subcategories of abelian (resp. exact) categories are $n$-abelian (resp. $n$-exact). These results allow to construct several examples of $n$-abelian and $n$-exact categories. Conversely, we prove that $n$-abelian categories satisfying certain mild assumptions can be realized as $n$-cluster-tilting subcategories of abelian categories. In analogy with a classical result of Happel, we show that the stable category of a Frobenius $n$-exact category has a natural $(n+2)$-angulated structure in the sense of Geiß-KellerOppermann. We give several examples of $n$-abelian and $n$-exact categories which have appeared in representation theory, commutative algebra, commutative and non-commutative algebraic geometry.
\end{abstract}

\section{Contents}

1. Introduction

2. Preliminary concepts 5

2.1. Conventions and notation 5

2.2. $n$-cokernels, $n$-kernels, and $n$-exact sequences 6

2.3. $n$-pushout diagrams and $n$-pullback diagrams 11

3. $n$-abelian categories 14

3.1. Definition and basic properties 14

3.2. Projective objects in $n$-abelian categories 19

3.3. $n$-abelian categories and cluster-tilting $\quad 22$

4. n-exact categories 29

4.1. Definition and basic properties 29

4.2. $n$-exact categories and cluster-tilting 36

5. Frobenius $n$-exact categories 40

5.1. Reminder on $(n+2)$-angulated categories 40

5.2. Frobenius $n$-exact categories and algebraic $(n+2)$-angulated categories 41

5.3. Standard construction 49

Date: December 8, 2015.

2010 Mathematics Subject Classification. Primary 18E99; Secondary 18E10, 18E30.

Key words and phrases. Abelian category; exact category; triangulated category; $n$-angulated category; homological algebra; cluster-tilting.

The author wishes to thank Erik Darpö, Laurent Demonet, Martin Herschend, Martin Kalck, Julian Külshammer, Boris Lerner, Yann Palu and Pierre-Guy Plamondon for motivating conversations regarding the contents of this article. This acknowledgment is extended to Prof. Osamu Iyama for his encouragement, helpful and interesting discussions, his comments on previous versions of this article, and especially for his generosity in sharing his ideas regarding a first definition of $n$-abelian category. Finally, the author wishes to express his sincere thanks to the anonymous referee for her/his detailed comments on a previous version of this article; in particular, for pointing out an error in earlier formulations of Theorem 3.20 and Lemma 3.22 
6. Examples 52

6.1. $n$-representation finite algebras 52

6.2. $n$-representation infinite algebras 53

6.3. Relative $n$-cluster-tilting subcategories 55

6.4. Isolated singularities

6.5. Algebraic $(n+2)$-angulated categories

References

\section{INTRODUCTION}

Let $n$ be a positive integer. In this article we introduce $n$-abelian and $n$-exact categories, these are higher analogs of abelian and exact categories from the viewpoint of higher homological algebra. Throughout we use the comparative adjective "higher" in relation to the length of exact sequences and not in the sense of higher category theory.

Abelian categories were introduced by Grothendieck in 24] to axiomatize the properties of the category of modules over a ring and of the category of sheaves over a scheme. It is often the case that interesting additive categories are not abelian but still have good homological properties with respect to a restricted class of short exact sequences. Exact categories were introduced by Quillen in [42] from this perspective to axiomatize extension-closed subcategories of abelian categories.

Derived categories play an important role in the study of the homological properties of abelian and exact categories. Their properties are captured by the notion of triangulated categories, introduced by Grothendieck-Verdier in 44. By a result of Happel, the stable category of a Frobenius exact category has a natural structure of a triangulated category, see [25, Thm. I.2.6]. Triangulated categories arising in this way have been called algebraic by Keller in [37. Algebraic triangulated categories have a natural $d g$-enhancement in the sense of Bondal-Kapranov [15], thus are often considered as a more reasonable class than that of general triangulated categories.

Recently, a new class of additive categories appeared in representation theory. The 2-cluster-tilting subcategories were introduced by Buan-Marsh-Reiten-ReinekeTodorov in [16] as the key concept involved in the additive categorification of the mutation combinatorics of Fomin-Zelevinsky's cluster algebras [19] via 2-CalabiYau triangulated categories. It was then observed by Iyama-Yoshino 34 that the notion of mutation can be extended to the class of $n$-cluster-tilting subcategories of triangulated categories.

From a different perspective, $n$-cluster-tilting subcategories of certain exact categories were introduced by Iyama in [30, and further investigated in [31, 29] from the viewpoint of higher Auslander-Reiten theory. In this theory, the notion of $n$ almost-split sequence, which are certain exact sequences with $n+2$ terms, plays an important role.

With motivation coming from these examples in representation theory, the class of $(n+2)$-angulated categories was introduced by Geiß-Keller-Oppermann as categories "naturally inhabited by the shadows of exact sequences with $n+2$ terms", to paraphrase the authors. We note that the case $n=1$ corresponds to triangulated categories. Their main source of examples of $(n+2)$-angulated categories are 
$n$-cluster-tilting subcategories of triangulated categories which are closed under the $n$-th power of the shift functor [21, Thm. 1]. The properties of $(n+2)$-angulated categories have been investigated by Bergh-Thaule in [12, 13, 14.

The aim of this article is to introduce $n$-abelian categories which are categories inhabited by certain exact sequences with $n+2$ terms, called $n$-exact sequences. The case $n=1$ corresponds to the classical concepts of abelian categories. We do so by modifying the axioms of abelian categories in a suitable manner. We prove several basic properties of $n$-abelian categories, including the existence of $n$-pushout (resp. $n$-pullback) diagrams which are analogs of classical pushout (resp. pullback) diagrams, see Theorem 3.8 .

An important source of examples of $n$-abelian categories are $n$-cluster-tilting subcategories. This is made precise by the following theorem.

Theorem 1 (see Theorem 3.16 for details). Let $\mathcal{M}$ be an $n$-cluster-tilting subcategory of an abelian category. Then $\mathcal{M}$ is an n-abelian category.

We introduce the notion of projective object in an $n$-abelian category, and study their properties. Remarkably, projective objects satisfy the following strong property which is obvious in the case of abelian categories.

Theorem 2 (see Theorem 3.12 for details). Let $\mathcal{M}$ be an $n$-abelian category and $P \in \mathcal{M}$ a projective object. Then, for every morphism $f: L \rightarrow M$ and every weak cokernel $g: M \rightarrow N$ of $f$, the following sequence is exact:

$$
\mathcal{M}(P, L) \stackrel{? \cdot f}{\longrightarrow} \mathcal{M}(P, M) \stackrel{? \cdot g}{\longrightarrow} \mathcal{M}(P, N) .
$$

Using this result, we show that certain $n$-abelian categories can be realized as $n$-cluster-tilting subcategories of abelian categories. More precisely, we prove the following theorem.

Theorem 3 (see Theorem 3.20 for details). Let $\mathcal{M}$ be a small projectively generated $n$-abelian category, and $\mathcal{P}$ the category of projective objects in $\mathcal{M}$. If $\bmod \mathcal{P}$ is injectively cogenerated, then $\mathcal{M}$ is equivalent to an n-cluster-tilting subcategory of $\bmod \mathcal{P}$.

After introducing $n$-abelian categories, it is natural to introduce $n$-exact categories as higher analogs of exact categories. For this, we modify Keller-Quillen's axioms of exact categories. We prove that the class of $n$-exact categories contains that of $n$-abelian categories, see Theorem 4.4. Similarly to the case of $n$-abelian categories, we prove the following theorem.

Theorem 4 (see Theorem 4.14 for details). Let $\mathcal{M}$ be an $n$-cluster-tilting subcategory of an exact category. Then $\mathcal{M}$ is an n-exact category.

We also introduce Frobenius $n$-exact categories. These are $n$-exact categories with enough projectives and enough injectives, and such that these two classes of objects coincide. Frobenius $n$-exact categories are related to $(n+2)$-angulated categories as shown by the following theorem.

Theorem 5 (see Theorem 5.11 for details). Let $\mathcal{M}$ be a Frobenius n-exact category. Then, the stable category $\underline{\mathcal{M}}$ has a natural structure of an $(n+2)$-angulated category.

Finally, we prove the following result also in the direction of Frobenius $n$-exact categories. 
Theorem 6 (see Theorem 5.16 for details). Let $\mathcal{M}$ be an $n$-cluster-tilting subcategory of a Frobenius exact category $\mathcal{E}$, and suppose that $\mathcal{M}$ is closed under taking $n$-th cosyzygies. Then, $\mathcal{M}$ is a Frobenius n-exact category.

This theorem is closely related to the results of Geiß-Keller-Oppermann. The relation between both approaches to construct $(n+2)$-angulated categories is explained in Theorem 5.16 .

Now we explain the notion of 2-exact category with concrete examples. The first example is due to Herschend-Iyama-Minamoto-Oppermann 27] (see also Theorem 6.6 and the example after it). Let $K$ be an algebraically closed field, coh $\mathbb{P}_{K}^{2}$ the category of coherent sheaves over the projective plane over $K$, and denote the category of vector bundles over $\mathbb{P}_{K}^{2}$ by vect $\mathbb{P}_{K}^{2}$. Note that vect $\mathbb{P}_{K}^{2}$ is closed under extensions in coh $\mathbb{P}_{K}^{2}$, and hence is an exact category. Then, the category

$$
\mathcal{U}:=\operatorname{add}\{\mathcal{O}(i) \mid i \in \mathbb{Z}\}
$$

of finite direct sums of degree shifts of the structure sheaf on $\mathbb{P}_{K}^{2}$ is a 2-clustertilting subcategory of vect $\mathbb{P}_{K}^{2}$. In view of the previous theorem, the category $\mathcal{U}$ is a 2-exact category. An interesting consequence of the 2-cluster-tilting property is that for every exact sequence

$$
0 \longrightarrow A \longrightarrow B \longrightarrow C \longrightarrow D \longrightarrow 0
$$

with terms in $\mathcal{U}$ the sequences of functors

$$
\begin{gathered}
\left.\left.\left.\left.0 \longrightarrow \operatorname{Hom}(-, A)\right|_{u} \longrightarrow \operatorname{Hom}(-, B)\right|_{u} \longrightarrow \operatorname{Hom}(-, C)\right|_{u} \longrightarrow \operatorname{Hom}(-, D)\right|_{u} \\
\left.\left.\left.\left.0 \longrightarrow \operatorname{Hom}(D,-)\right|_{u} \longrightarrow \operatorname{Hom}(C,-)\right|_{u} \longrightarrow \operatorname{Hom}(B,-)\right|_{u} \longrightarrow \operatorname{Hom}(A,-)\right|_{u}
\end{gathered}
$$

are exact. In general, we call a sequence of the form (1.1) satisfying these properties a 2-exact sequence. In this case, the Koszul complexes

$$
0 \longrightarrow \mathcal{O}(i-3) \longrightarrow \mathcal{O}(i-2)^{3} \longrightarrow \mathcal{O}(i-1)^{3} \longrightarrow \mathcal{O}(i) \longrightarrow 0 \quad(i \in \mathbb{Z})
$$

gives a special class of 2-exact sequences called 2-almost-split sequences in higher Auslander-Reiten theory.

Let us provide the reader with another example of a 2-exact category, following Iyama [30, Sec. 2.5]. Let $K$ be an algebraically closed field and $S:=K \llbracket x_{0}, x_{1}, x_{2} \rrbracket$ be the ring of power series three commuting variables. Also, let $G$ be a finite subgroup of $\mathrm{SL}_{3}(K)$ and $R:=S^{G}$ the associated invariant subring of $S$. Finally, we denote the category of Cohen-Macaulay $R$-modules by CM $R$, see Section 6.4 for details and definitions. Note that $\mathrm{CM} R$ is a Frobenius exact category. Then, the category

$$
\mathcal{S}:=\operatorname{add} S=\left\{M \in \mathrm{CM} R \mid M \text { is a direct summand of } S^{m} \text { for some } m\right\}
$$

is a 2-cluster-tilting subcategory of $\mathrm{CM} R$, i.e. we have

$$
\mathcal{S}=\left\{M \in \mathrm{CM} R \mid \operatorname{Ext}_{R}^{1}(\mathcal{S}, M)=0\right\}=\left\{M \in \mathrm{CM} R \mid \operatorname{Ext}_{R}^{1}(M, \mathcal{S})=0\right\} .
$$

An important example of a 2-exact sequence in this case is given by the Koszul complex of $S$ :

$$
K(S): 0 \longrightarrow S \longrightarrow S^{3} \longrightarrow S^{3} \longrightarrow S \longrightarrow K \longrightarrow
$$


As a complex of $R$-modules, $K(S)$ is the direct sum of finitely many 2-almost-split sequences and a 2 -fundamental sequence in the sense of [30, Sec. 3].

Let us mention other examples of $n$-cluster-tilting subcategories, which give us examples of $n$-abelian and $n$-exact categories.

Finite dimensional algebras of finite global dimension whose module category contains an $n$-cluster-tilting subcategory are one of the central objects of study of higher Auslander-Reiten theory. A distinguished class of such algebras, the socalled $n$-representation-finite algebras, were introduced by Iyama-Oppermann in 32 and have been studied in greater detail by Herschend-Iyama in the case $n=2$, see [26].

In a parallel direction, 2-cluster-tilting subcategories of the module category of a preprojective algebra of Dynkin type, which has infinite global dimension, are central in Geiß-Leclerc-Schröer's categorification of cluster algebras arising in Lie theory, see 23] and the references therein.

Further examples of $n$-cluster-tilting subcategories of abelian and exact categories have been constructed by Amiot-Iyama-Reiten in the category of CohenMacaulay modules over an isolated singularity [1].

Finally, let us give a brief description of the contents of this article. In Section 2 we introduce the basic concepts behind the definitions of $n$-abelian and $n$-exact categories: $n$-cokernels, $n$-kernels, $n$-exact sequences, and $n$-pushout and $n$-pullback diagrams (the reader will forgive the author for his lack of inventiveness in naming these concepts). The class of $n$-abelian categories is introduced in Section 3 , where we also give a characterization of semisimple categories in terms of $n$-abelian categories. In Theorems 3.16 and 3.20 we explore the connection between $n$-abelian categories and $n$-cluster-tilting subcategories of abelian categories. Later, in Section

4 we introduce $n$-exact categories and establish a connection with $n$-cluster-tilting subcategories of exact categories in Theorem 4.14. Frobenius $n$-exact categories and their main properties are introduced in Section 5 . At last, in Section 6 we provide several examples to illustrate our results.

\section{Preliminary CONCEPtS}

We begin by fixing our conventions and notation, and by reminding the reader of basic concepts in homological algebra that we use freely in the remainder.

2.1. Conventions and notation. Throughout this article $n$ always denotes a fixed positive integer. Let $\mathcal{C}$ be a category; all subcategories considered are supposed to be full. If $A, B \in \mathcal{C}$, then we denote the set of morphisms $A \rightarrow B$ in $\mathcal{C}$ by $\mathcal{C}(A, B)$. We denote the identity morphism of an object $C \in \mathcal{C}$ by $1=1_{C}$. We denote composition of morphisms by concatenation: if $f \in \mathcal{C}(A, B)$ and $g \in \mathcal{C}(B, C)$, then $f g \in \mathcal{C}(A, C)$. If $F: \mathcal{C} \rightarrow \mathcal{D}$ is a functor, then the essential image of $F$ is the full subcategory of $\mathcal{D}$ given by

$$
F \mathcal{C}:=\{D \in \mathcal{D} \mid \exists C \in \mathcal{C} \text { such that } F C \cong D\} .
$$

A morphism $e \in \mathcal{C}(A, A)$ is idempotent if $e^{2}=e$. We say that $\mathcal{C}$ is idempotent complete if for every idempotent $e \in \mathcal{C}(A, A)$ there exist an object $B$ and morphisms $r \in \mathfrak{C}(A, B)$ and $s \in \mathcal{C}(B, A)$ such that $r s=e$ and $s r=1_{B}$.

Let $\mathcal{C}$ be an additive category in the sense of [45, Sec. A.4.1]. If $X$ is a class of objects in $\mathcal{C}$, then we denote by add $\mathcal{X}$ the full subcategory whose objects are direct summands of direct sums of objects in $X$. 
We denote the category of (cochain) complexes in $\mathcal{C}$ by $\mathrm{Ch}(\mathcal{C})$. Also, we denote the full subcategory of $\mathrm{Ch}(\mathcal{C})$ given by all complexes concentrated in non-negative (resp. non-positive) cohomological degrees by $\mathrm{Ch}^{\geq 0}(\mathcal{C})\left(\right.$ resp. $\mathrm{Ch}^{\leq 0}(\mathcal{C})$ ). For convenience, we denote by $\mathrm{Ch}^{n}(\mathcal{C})$ the full subcategory of $\mathrm{Ch}(\mathcal{C})$ given by all complexes

$$
X^{0} \stackrel{d^{0}}{\longrightarrow} X^{1} \stackrel{d^{1}}{\longrightarrow} \cdots \stackrel{d^{n-1}}{\longrightarrow} X^{n} \stackrel{d^{n}}{\longrightarrow} X^{n+1}
$$

which are concentrated in degrees $0,1, \ldots, n+1$. A morphism of complexes

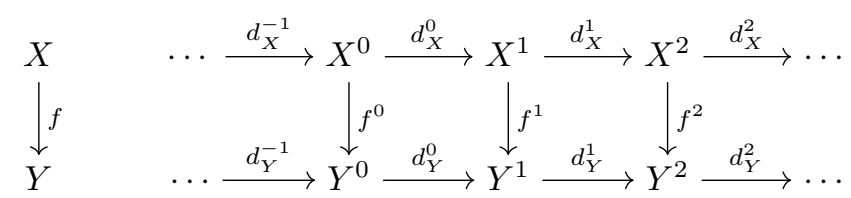

is null-homotopic if for all $k \in \mathbb{Z}$ there exists a morphism $h^{k}: X^{k} \rightarrow Y^{k-1}$ such that

$$
f^{k}=h^{k} d_{Y}^{k-1}+d_{X}^{k} h^{k+1} .
$$

In this case we say that $h=\left(h^{k} \mid k \in \mathbb{Z}\right)$ is a null-homotopy. We say that two morphisms of complexes $f: X \rightarrow Y$ and $g: X \rightarrow Y$ are homotopic if their difference is null-homotopic. A homotopy between $f$ and $g$ is a null-homotopy $h$ of $f-g$ and we write $h: f \rightarrow g$. It is easily verified that being homotopic induces an equivalence relation on $\mathrm{Ch}(\mathrm{C})(X, Y)$. The homotopy category of $\mathcal{C}$, denoted by $\mathrm{H}(\mathcal{C})$, is the category with the same objects as $\mathrm{Ch}(\mathcal{C})$ and in which morphisms are given by morphisms of complexes modulo homotopy. For further information on chain complexes and the homotopy category we refer the reader to [45, Ch. 1].

We remind the reader of the notion of functorially finite subcategory of an additive category. Let $\mathcal{C}$ be an additive category and $\mathcal{D}$ a (full) subcategory of $\mathcal{C}$. We say that $\mathcal{D}$ is covariantly finite in $\mathcal{C}$ if for every $C \in \mathcal{C}$ there exists an object $D \in \mathcal{D}$ and a morphism $f: C \rightarrow D$ such that, for all $D^{\prime} \in \mathcal{D}$, the sequence of abelian groups

$$
\mathcal{C}\left(D, D^{\prime}\right) \stackrel{f \cdot ?}{\longrightarrow} \mathcal{C}\left(C, D^{\prime}\right) \longrightarrow 0
$$

is exact. Such a morphism $f$ is called a left $\mathcal{D}$-approximation of $C$. The notions of contravariantly finite subcategory of $\mathcal{C}$ and right $\mathcal{D}$-approximation are defined dually. A functorially finite subcategory of $\mathcal{C}$ is a subcategory which is both covariantly and contravariantly finite in $\mathcal{C}$. For further information on functorially finite subcategories we refer the reader to [7, 6].

2.2. $n$-cokernels, $n$-kernels, and $n$-exact sequences. Let $\mathcal{C}$ be an additive category and $f: A \rightarrow B$ a morphism in $\mathcal{C}$. A weak cokernel of $f$ is a morphism $g: B \rightarrow C$ such that for all $C^{\prime} \in \mathcal{C}$ the sequence of abelian groups

$$
\mathcal{C}\left(C, C^{\prime}\right) \stackrel{g \cdot ?}{\longrightarrow} \mathcal{C}\left(B, C^{\prime}\right) \stackrel{f \cdot ?}{\longrightarrow} \mathcal{C}\left(A, C^{\prime}\right)
$$

is exact. Equivalently, $g$ is a weak cokernel of $f$ if $f g=0$ and for each morphism $h: B \rightarrow C^{\prime}$ such that $f h=0$ there exists a (not necessarily unique) morphism $p: C \rightarrow C^{\prime}$ such that $h=g p$. These properties are subsumed in the following 
commutative diagram:

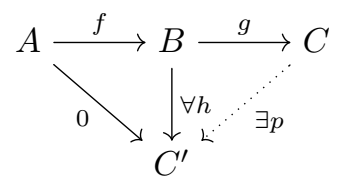

Clearly, a weak cokernel $g$ of $f$ is a cokernel of $f$ if and only if $g$ is an epimorphism. The concept of weak kernel is defined dually.

The following general result, together with its dual, plays a central role in the sequel.

Comparison Lemma 2.1. Let $\mathcal{C}$ be an additive category and $X \in \mathrm{Ch}^{\geq 0}(\mathcal{C})$ a complex such that for all $k \geq 0$ the morphism $d_{X}^{k+1}$ is a weak cokernel of $d_{X}^{k}$. If $f: X \rightarrow Y$ and $g: X \rightarrow Y$ are morphisms in $\mathrm{Ch}^{\geq 0}(\mathrm{C})$ such that $f^{0}=g^{0}$, then there exists a homotopy $h: f \rightarrow g$ such that $h^{1}$ is the zero morphism.

Proof. Let $u:=f-g$ and for all $k \leq 1$ let $h^{k}: X^{k} \rightarrow Y^{k-1}$ be the zero morphism. Note that $u^{0}=0$ by hypothesis. We proceed by induction on $k$. Let $k \geq 1$ and suppose that for all $\ell \leq k$ we have constructed a morphism

$$
h^{\ell}: X^{\ell} \rightarrow Y^{\ell-1}
$$

such that

$$
u^{\ell-1}=h^{\ell-1} d_{Y}^{\ell-2}+d_{X}^{\ell-1} h^{\ell} .
$$

Since $u$ is a morphism of complexes, we have

$$
\begin{aligned}
d_{X}^{k-1}\left(u^{k}-h^{k} d_{Y}^{k-1}\right) & =d_{X}^{k-1} u^{k}+\left(h^{k-1} d_{Y}^{k-2}-u^{k-1}\right) d_{Y}^{k-1} \\
& =d_{X}^{k-1} u^{k}-u^{k-1} d_{Y}^{k-1} \\
& =0 .
\end{aligned}
$$

Hence, given that $d_{X}^{k}$ is a weak cokernel of $d_{X}^{k-1}$, there exists a morphism

$$
h^{k+1}: X^{k+1} \rightarrow Y^{k}
$$

such that $u^{k}-h^{k} d_{Y}^{k-1}=d_{X}^{k} h^{k+1}$ or, equivalently,

$$
u^{k}=h^{k} d_{Y}^{k-1}+d_{X}^{k} h^{k+1} .
$$

This finishes the construction of the required null-homotopy $h: f-g \rightarrow 0$.

The following terminology will prove convenient in the sequel.

Definition 2.2. Let $\mathcal{C}$ be an additive category and $d^{0}: X^{0} \rightarrow X^{1}$ a morphism in C. An $n$-cokernel of $d^{0}$ is a sequence

$$
\left(d^{1}, \ldots, d^{n}\right): X^{1} \stackrel{d^{1}}{\longrightarrow} X^{2} \stackrel{d^{2}}{\longrightarrow} \cdots \stackrel{d^{n}}{\longrightarrow} X^{n+1}
$$

such that for all $Y \in \mathcal{C}$ the induced sequence of abelian groups

$$
0 \longrightarrow \mathrm{C}\left(X^{n+1}, Y\right) \stackrel{d^{n} \cdot ?}{\longrightarrow} \mathrm{C}\left(X^{n}, Y\right) \stackrel{d^{n-1} \cdot ?}{\longrightarrow} \cdots \stackrel{d^{1} \cdot ?}{\longrightarrow} \mathrm{C}\left(X^{1}, Y\right) \stackrel{d^{0} \cdot ?}{\longrightarrow} \mathcal{C}\left(X^{0}, Y\right)
$$

is exact. Equivalently, the sequence $\left(d^{1}, \ldots, d^{n}\right)$ is an $n$-cokernel of $d^{0}$ if for all $1 \leq k \leq n-1$ the morphism $d^{k}$ is a weak cokernel of $d^{k-1}$, and $d^{n}$ is moreover a cokernel of $d^{n-1}$. The concept of $n$-kernel of a morphism is defined dually. 
Remark 2.3. If $n \geq 2$, then $n$-cokernels are not unique in general. Indeed, for each object $C \in \mathcal{C}$ the sequence $0 \rightarrow C \stackrel{1}{\rightarrow} C$ is a 2-cokernel of the morphism $0 \rightarrow 0$. This shortcoming can be resolved if one considers $n$-cokernels up to isomorphism in $\mathrm{H}(\mathcal{C})$, see Proposition 2.7 .

As explained in the Introduction, $n$-exact sequences, defined below, are the object of study of higher homological algebra. The investigation of their properties is our main concern for the rest of this article.

Definition 2.4. Let $\mathcal{C}$ be an additive category. An $n$-exact sequence in $\mathcal{C}$ is a complex

$$
X^{0} \stackrel{d^{0}}{\longrightarrow} X^{1} \stackrel{d^{1}}{\longrightarrow} \cdots \stackrel{d^{n-1}}{\longrightarrow} X^{n} \stackrel{d^{n}}{\longrightarrow} X^{n+1}
$$

in $\mathrm{Ch}^{n}(\mathcal{C})$ such that $\left(d^{0}, \ldots, d^{n-1}\right)$ is an $n$-kernel of $d^{n}$, and $\left(d^{1}, \ldots, d^{n}\right)$ is an $n$-cokernel of $d^{0}$.

Let $\mathcal{C}$ be an additive category. We remind the reader that a complex $X \in \mathrm{Ch}(\mathcal{C})$ is contractible if the identity morphism of $X$ is null-homotopic or, equivalently, $X$ is isomorphic to the zero complex in $\mathrm{H}(\mathcal{C})$. As a first analogy with the classical theory, let us show that the class of $n$-exact sequences is closed under isomorphisms in $\mathrm{H}(\mathrm{C})$.

Proposition 2.5. Let $\mathcal{C}$ be an additive category and $X$ and $Y$ be complexes in $\mathrm{Ch}^{n}(\mathcal{C})$ which are isomorphic in $\mathrm{H}(\mathcal{C})$. Then the following statements hold.

(i) The complex $X$ is an n-exact sequence if and only if $Y$ is an n-exact sequence.

(ii) Every contractible complex with $n+2$ terms is an n-exact sequence.

Proof. Note that the second claim follows immediately from the first one since the zero complex in $\mathrm{Ch}^{n}(\mathrm{C})$ is clearly an $n$-exact sequence. Suppose that $X$ is an $n$-exact sequence. By hypothesis, there exist morphisms of complexes

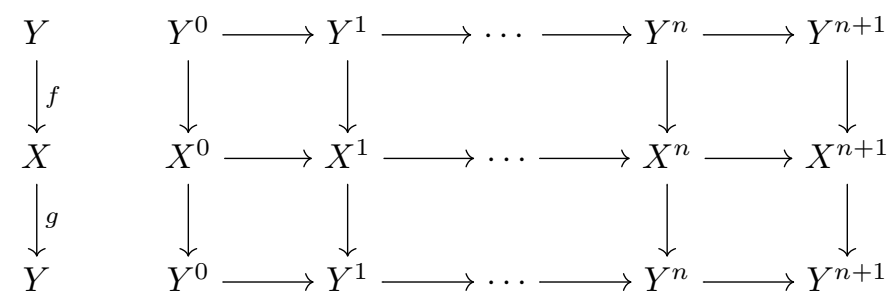

together with a homotopy $h: f g \rightarrow 1_{Y}$. Hence, for all $k \in\{1, \ldots, n\}$ we have

$$
1_{Y^{k}}=f^{k} g^{k}-h^{k} d_{Y}^{k-1}-d_{Y}^{k} h^{k+1} .
$$

In particular, we have

$$
1_{Y^{n+1}}=f^{n+1} g^{n+1}-h^{n+1} d_{Y}^{n} .
$$

We claim that for all $k \in\{1, \ldots, n\}$ the morphism $d_{Y}^{k}$ is a weak cokernel of $d_{Y}^{k-1}$. Indeed, let $k \in\{1, \ldots, n\}$ and $u: Y^{k} \rightarrow C$ be a morphism such that $d_{Y}^{k-1} u=0$. It follows that

$$
\left(d_{X}^{k-1} g^{k}\right) u=g^{k-1}\left(d_{Y}^{k-1} u\right)=0 .
$$


Since $d_{X}^{k}$ is a weak cokernel of $d_{X}^{k-1}$ there exists a morphism $v: X^{k+1} \rightarrow C$ such that $g^{k} u=d_{X}^{k} v$. Therefore,

$$
f^{k}\left(g^{k} u\right)=\left(f^{k} d_{X}^{k}\right) v=d_{Y}^{k} f^{k+1} v .
$$

By composing the identity (2.1) on the right with $u$ and substituting the identity (2.3) we obtain

$$
u=\left(f^{k} g^{k}\right) u-\left(d_{Y}^{k} h^{k+1}\right) u=d_{Y}^{k}\left(f^{k+1} v-h^{k+1} u\right) .
$$

Therefore $u$ factors through $d_{Y}^{k}$. This shows that $d_{Y}^{k}$ is a weak kernel of $d_{Y}^{k-1}$.

We need to show that $d_{Y}^{n}$ is moreover a cokernel of $d_{Y}^{n-1}$. For this it is enough to show that $d_{Y}^{n}$ is an epimorphism for we already know that it is a weak cokernel of $d_{Y}^{n-1}$. Let $w: Y^{n+1} \rightarrow C$ be a morphism such that $d_{Y}^{n} w=0$. It follows that

$$
d_{X}^{n}\left(g^{n+1} w\right)=g^{n}\left(d_{Y}^{n} w\right)=0 .
$$

Given that $d_{X}^{n}$ is an epimorphism we deduce that $g^{n+1} w=0$. By composing 2.2 . on the right with $w$, we obtain

$$
w=f^{n+1}\left(g^{n+1} w\right)-h^{n+1}\left(d_{Y}^{n} w\right)=0 .
$$

Therefore $d_{Y}^{n}$ is an epimorphism. This shows that $\left(d_{Y}^{1}, \ldots, d_{Y}^{n}\right)$ is an $n$-cokernel of $d_{Y}^{0}$. By duality, the sequence $\left(d_{Y}^{0}, \ldots, d_{Y}^{n-1}\right)$ is an $n$-kernel of $d_{Y}^{n}$. Hence $Y$ is an $n$-exact sequence. The converse implication is analogous.

We have the following useful characterization of contractible $n$-exact sequences.

Proposition 2.6. Let $\mathcal{C}$ be an additive category and $X$ a complex in $\mathrm{Ch}^{n}(\mathcal{C})$ such that $\left(d^{1}, \ldots, d^{n}\right)$ is an n-cokernel of $d^{0}$. Then, $d^{0}$ is a split monomorphism if and only if $X$ is a contractible $n$-exact sequence.

Proof. Suppose that $d^{0}$ is a split monomorphism. Hence there exists a morphism $h^{1}: X^{1} \rightarrow X^{0}$ such that $d^{0} h^{1}=1_{X^{0}}$. We shall extend $h^{1}$ to a null-homotopy of $1_{X}$. Inductively, let $k \in\{0,1, \ldots, n\}$ and suppose that for all $\ell \leq k$ we have constructed a morphism $h^{\ell}: X^{\ell} \rightarrow X^{\ell-1}$ such that

$$
1_{X^{\ell-1}}=h^{\ell-1} d^{\ell-2}+d^{\ell-1} h^{\ell} .
$$

Composing this identity, for $\ell=k$, on the left with $d^{k-1}$ we obtain

$$
d^{k-1}=\left(h^{k-1} d^{k-2}+d^{k-1} h^{k}\right) d^{k-1}=d^{k-1}\left(h^{k} d^{k-1}\right) .
$$

Since $d^{k}$ is a weak cokernel of $d^{k-1}$, there exists a morphism $h^{k+1}: X^{k+1} \rightarrow X^{k}$ such that $d^{k} h^{k+1}=1_{X^{k}}-h^{k} d^{k-1}$ or, equivalently,

$$
1_{X^{k}}=h^{k} d^{k-1}+d^{k} h^{k+1} .
$$

This finishes the induction step. It remains to show that $1_{X^{n+1}}=h^{n} d^{n}$. For this, let $k=n$ and note that composing the previous equality on the right by $d^{n}$ yields

$$
d^{n}=\left(h^{n} d^{n-1}+d^{n} h^{n+1}\right) d^{n}=d^{n}\left(h^{n+1} d^{n}\right) .
$$

Since $d^{n}$ is an epimorphism, we have $1_{X^{n+1}}=h^{n} d^{n}$, which is what we needed to show. This shows that $X$ is a contractible complex, and so it is also an $n$-exact sequence. The converse implication is obvious.

The following result implies that $n$-cokernels and $n$-kernels are unique up to isomorphism in $\mathrm{H}(\mathcal{C})$. 
Proposition 2.7. Let $\mathcal{C}$ be an additive category and $f: X \rightarrow Y$ a morphism of $n$-exact sequences in $\mathcal{C}$ such that $f^{k}$ and $f^{k+1}$ are isomorphisms for some $k \in$ $\{1, \ldots, n\}$. Then, $f$ induces an isomorphism in $\mathrm{H}(\mathrm{C})$.

Proof. Using the factorization property of weak cokernels and weak kernels we can construct a morphism of $n$-exact sequences $g: Y \rightarrow X$ where $g^{k}$ and $g^{k+1}$ are the inverses of $f^{k}$ and $f^{k+1}$ respectively:

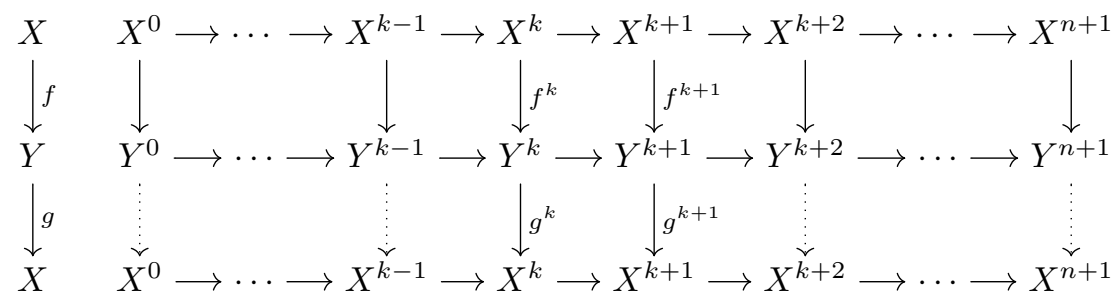

Then, the Comparison Lemma 2.1 and its dual applied to diagrams

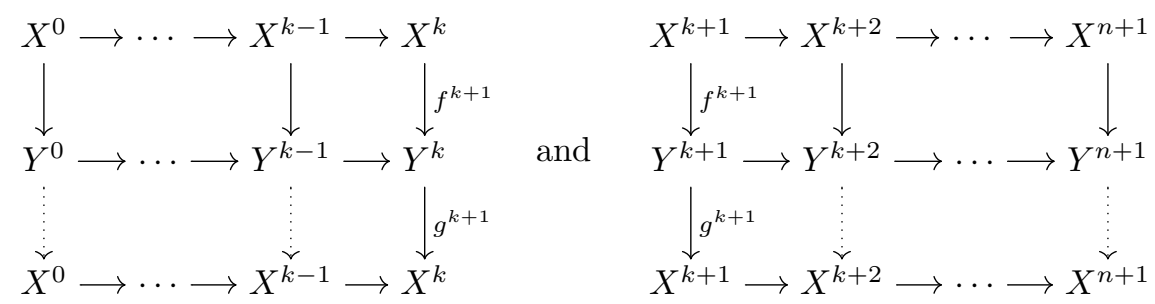

respectively imply that $f$ and $g$ induce mutually inverse isomorphisms in the homotopy category $\mathrm{H}(\mathcal{C})$.

Remark 2.8. The statement of Proposition 2.7 can be interpreted as saying that each morphism in an $n$-exact sequence determines the others "up to homotopy". To prove that equivalences of $n$-exact sequences also induce isomorphisms in $\mathrm{H}(\mathcal{C})$ we need to impose a richer structure on the category $\mathcal{C}$, see Proposition 4.10

Definition 2.9. Let $\mathcal{C}$ be an additive category. A morphism of $n$-exact sequences in $\mathcal{C}$ is a morphism of complexes

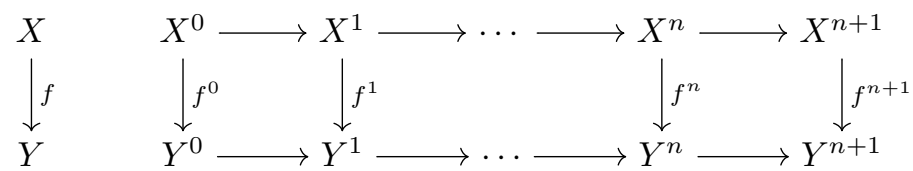

in which each row is an $n$-exact sequence. We say that $f$ is an equivalence if $f^{0}=1_{X^{0}}$ and $f^{n+1}=1_{X^{n+1}}$.

Remark 2.10. In Proposition 4.10 (in which we consider morphisms of complexes up to homotopy) we show that, in the case of $n$-exact categories, equivalences of $n$ exact sequences are in fact an equivalence relation on the class of $n$-exact sequences. 
2.3. $n$-pushout diagrams and $n$-pullback diagrams. Let $\mathcal{C}$ be an additive category. A pushout diagram of a pair of morphisms

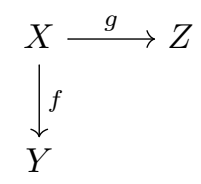

in $\mathcal{C}$ can be identified with a cokernel of the morphism $[-g f]^{\top}: X \rightarrow Z \oplus Y$. This motivates us to introduce the following concept.

Definition 2.11. Let $\mathcal{C}$ be an additive category, $X$ a complex in $\mathrm{Ch}^{n-1}(\mathcal{C})$, and $f^{0}: X^{0} \rightarrow Y^{0}$ a morphism in $\mathcal{C}$. An $n$-pushout diagram of $X$ along $f^{0}$ is a morphism of complexes



such that in the mapping cone $C=C(f)$

$$
X^{0} \stackrel{d_{C}^{-1}}{\longrightarrow} X^{1} \oplus Y^{0} \stackrel{d_{C}^{0}}{\longrightarrow} \cdots \stackrel{d_{C}^{n-2}}{\longrightarrow} X^{n} \oplus Y^{n-1} \stackrel{d_{C}^{n-1}}{\longrightarrow} Y^{n} .
$$

the sequence $\left(d_{C}^{0}, \ldots, d_{C}^{n-1}\right)$ is an $n$-cokernel of $d_{C}^{-1}$, where we define

$$
d_{C}^{k}:=\left[\begin{array}{cc}
-d_{X}^{k+1} & 0 \\
f^{k+1} & d_{Y}^{k}
\end{array}\right]: X^{k+1} \oplus Y^{k} \longrightarrow X^{k+2} \oplus Y^{k+1}
$$

for each $k \in\{-1,0,1, \ldots, n-1\}$. In particular,

$$
d_{C}^{-1}=\left[\begin{array}{c}
-d_{X}^{0} \\
f^{0}
\end{array}\right] \quad \text { and } \quad d_{C}^{n-1}=\left[\begin{array}{ll}
f^{n} & d_{Y}^{n-1}
\end{array}\right] .
$$

Note that the fact that $C(f)$ is a complex encodes precisely that $X$ and $Y$ are complexes and that $f$ is a morphism of complexes. The concept of $n$-pullback diagram is defined dually.

We now state some of general properties of $n$-pushout diagrams.

Proposition 2.12. Let $\mathcal{C}$ be an additive category. Suppose that we are given an n-pushout diagram

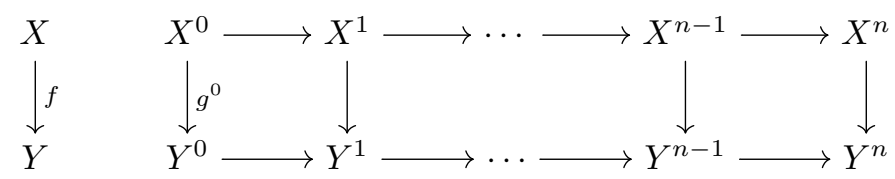

and let $k \in\{0,1, \ldots, n-2\}$. If $d_{Y}^{k+1}$ is a weak cokernel of $d_{Y}^{k}$, then $d_{X}^{k+1}$ is a weak cokernel of $d_{X}^{k}$. 
Proof. Put $C:=C(f)$ and let $u: X^{k+1} \rightarrow M$ be a morphism such that $d_{X}^{k} u=0$. Consider the solid part of the following commutative diagram:

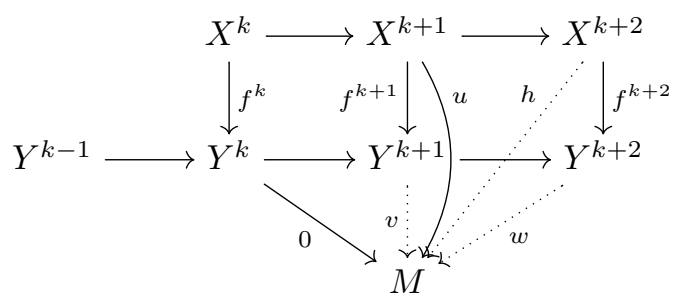

Given that $d_{C}^{k}: X^{k+1} \oplus Y^{k} \rightarrow X^{k+2} \oplus Y^{k+1}$ is a weak cokernel of $d_{C}^{-1}: X^{k} \oplus Y^{k-1} \rightarrow$ $X^{k+1} \oplus Y^{k}$, there exist morphisms $v: Y^{k+1} \rightarrow M$ and $h: X^{k+2} \rightarrow M$ such that $d_{Y}^{k} v=0$ and $u-f^{k+1} v=d_{X}^{k+1} h$. Since $d_{Y}^{k+1}$ is a weak cokernel of $d_{Y}^{k}$ there exists a morphism $w: Y^{k+2} \rightarrow M$ such that $v=d_{Y}^{k+1} w$. Therefore we have

$$
\begin{aligned}
u & =d_{X}^{k+1} h+f^{k+1} v \\
& =d_{X}^{k+1} h+f^{k+1}\left(d_{Y}^{k+1} w\right) \\
& =d_{X}^{k+1}\left(h+f^{k+2} w\right) .
\end{aligned}
$$

This shows that $d_{X}^{k+1}$ is a weak cokernel of $d_{X}^{k}$.

Our choice of terminology in Definition 2.11 is justified by the following property.

Proposition 2.13. Let $\mathcal{C}$ be an additive category, $g: X \rightarrow Z$ a morphism of complexes in $\mathrm{Ch}^{n-1}(\mathrm{C})$ and suppose there exists an n-pushout diagram of $X$ along $g^{0}$

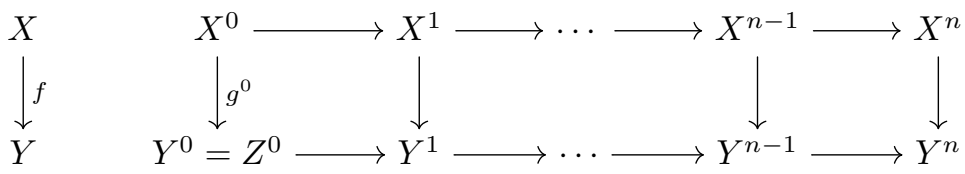

Then, there exists a morphism of complexes $p: Y \rightarrow Z$ such that $p^{0}=1_{Z^{0}}$ and a homotopy $h: f p \rightarrow g$ with $h^{1}=0$. Moreover, these properties determine $p$ uniquely up to homotopy.

Proof. Let $h^{1}: X^{1} \rightarrow Z^{0}$ be the zero morphism, $p^{0}=1_{Z^{0}}$ and $C:=C(f)$. Inductively, suppose that $0 \leq k \leq n$ and that for all $\ell \leq k$ we have constructed a morphism $p^{\ell}: Y^{\ell} \rightarrow Z^{\ell}$ such that

$$
d_{Y}^{\ell-1} p^{\ell}=p^{\ell-1} d_{Z}^{\ell-1}
$$

and a morphism $h^{\ell+1}: X^{\ell+1} \rightarrow Z^{\ell}$ such that

$$
f^{\ell} p^{\ell}-g^{\ell}=h^{\ell} d_{Z}^{\ell-1}+d_{X}^{\ell} h^{\ell+1} .
$$

We claim that the composition

$$
X^{k} \oplus Y^{k-1} \stackrel{\left[\begin{array}{cc}
-d_{X}^{k} & 0 \\
f^{k} & d_{Y}^{k-1}
\end{array}\right]}{\longrightarrow} X^{k+1} \oplus Y^{\left.k \stackrel{\left[g^{k+1}-h^{k+1} d_{Z}^{k}\right.}{~} p^{k} d_{Z}^{k}\right]} Z^{k+1}
$$

vanishes. Indeed, on one hand we have

$$
f^{k}\left(p^{k} d_{Z}^{k}\right)=\left(g^{k}+d_{X}^{k} h^{k+1}\right) d_{Z}^{k}=d_{X}^{k}\left(g^{k+1}-h^{k+1} d_{Z}^{k}\right) .
$$


On the other hand, we have

$$
d_{Y}^{k-1}\left(p^{k} d_{Z}^{k}\right)=p^{k-1} d_{Z}^{k-1} d_{Z}^{k}=0 .
$$

The claim follows.

Next, since $d_{C}^{k}$ is a weak cokernel of $d_{C}^{k-1}$, there exists a morphism $p^{k+1}: Y^{k+1} \rightarrow$ $Z^{k+1}$ such that

$$
d_{Y}^{k} p^{k+1}=p^{k} d_{Z}^{k}
$$

and a morphism $h^{k+2}: X^{k+2} \rightarrow Y^{k+1}$ such that

$$
g^{k+1}+h^{k+1} d_{Z}^{k}=-d_{X}^{k+1} h^{k+2}+f^{k+1} p^{k+1} .
$$

This finishes the induction step, and the construction of the required morphism $p: Y \rightarrow Z$. Moreover, $h: f p \rightarrow g$ is a homotopy (note that $h^{n+1}=0$ ). The last claim follows immediately from the Comparison Lemma 2.1

Definition-Proposition 2.14. Let $\mathcal{C}$ be an additive category and $g^{0}: X^{0} \rightarrow Z^{0}$ a morphism in $\mathcal{C}$. Suppose that there exists an n-pushout diagram of $X \in \mathrm{Ch}^{n-1}(\mathcal{C})$ along $g^{0}$

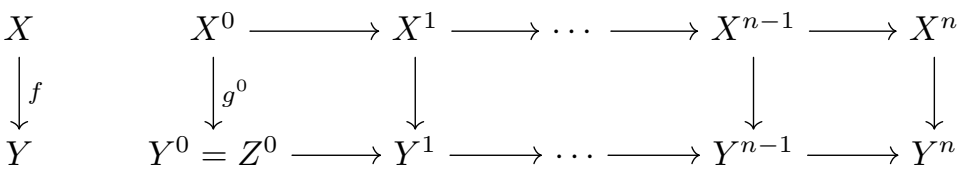

Then, the following statements hold:

(i) There exists an n-pushout diagram $\tilde{f}: X \rightarrow \tilde{Y}$ of $X$ along $g^{0}$ such that for every morphism $g: X \rightarrow Z$ of complexes lifting $g^{0}$ and such that $Z \in$ $\mathrm{Ch}^{n-1}(\mathrm{C})$ there exists a morphism of complexes $p: Y \rightarrow Z$ such that $p^{0}=$ $1_{Z^{0}}$ and $\tilde{f} p=g$.

(ii) For each $2 \leq k \leq n$ the morphism $\tilde{f}^{k}$ is a split monomorphism.

(iii) We have $\tilde{Y}=Y \oplus X^{\prime}$ for a contractible complex $X^{\prime} \in \mathrm{Ch}^{n-1}(\mathrm{C})$.

We call the morphism $\tilde{f}: X \rightarrow \tilde{Y}$ a good $n$-pushout diagram of $X$ along $g^{0}$.

Proof. If $n=1$ the result is trivial, so we may assume that $n \geq 2$. For $C \in \mathcal{C}$ and $k \in \mathbb{Z}$, let $i_{k}(C)$ be the complex with $d^{k}=1_{C}$ and which is 0 in each degree different from $k$ and $k+1$. We define

$$
X^{\prime}:=\bigoplus_{k=2}^{n} i_{k-1}\left(X^{k}\right)
$$

and $\tilde{Y}:=Y \oplus X^{\prime}$. Note that $\tilde{Y}^{0}=Y^{0}$ and that $X^{\prime}$ is a contractible complex. It readily follows that the diagram

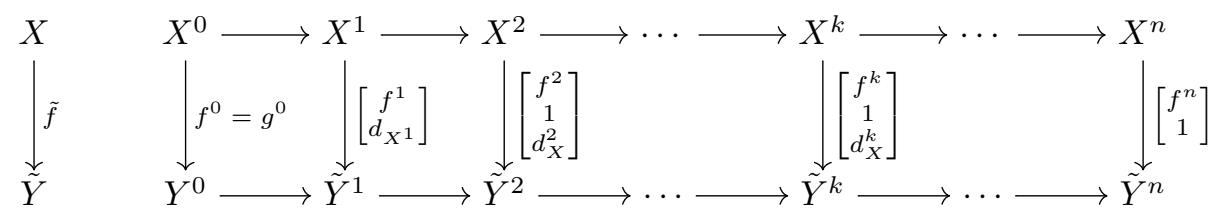

commutes. Observe that for each $2 \leq k \leq n$ the morphism $\tilde{f}^{k}$ is a split monomorphism. Using Proposition 2.13 it is easy to show that $\tilde{f}$ has the required factorization property; the details are left to the reader. 


\section{3. n-ABELIAN CATEGORIES}

In this section we introduce $n$-abelian categories and establish their basic properties; we give a characterization of semisimple categories in terms of $n$-abelian categories. We also introduce projective objects in $n$-abelian categories and study their basic properties. Finally, we show that $n$-cluster-tilting subcategories of abelian categories are $n$-abelian; we give a partial converse in the case of $n$-abelian categories with enough projectives.

3.1. Definition and basic properties. The following definition is motivated by the axioms of abelian categories given in [45, Def. A.4.2].

Definition 3.1. Let $n$ be a positive integer. An $n$-abelian category is an additive category $\mathcal{M}$ which satisfies the following axioms:

(A0) The category $\mathcal{M}$ is idempotent complete.

(A1) Every morphism in $\mathcal{M}$ has an $n$-kernel and an $n$-cokernel.

(A2) For every monomorphism $f^{0}: X^{0} \rightarrow X^{1}$ in $\mathcal{N}$ and for every $n$-cokernel $\left(f^{1}, \ldots, f^{n}\right)$ of $f^{0}$, the following sequence is $n$-exact:

$$
X^{0} \stackrel{f^{0}}{\longrightarrow} X^{1} \stackrel{f^{1}}{\longrightarrow} \cdots \stackrel{f^{n-1}}{\longrightarrow} X^{n} \stackrel{f^{n}}{\longrightarrow} X^{n+1} .
$$

$\left(\mathrm{A} 2^{\mathrm{op}}\right)$ For every epimorphism $g^{n}: X^{n} \rightarrow X^{n+1}$ in $\mathcal{M}$ and for every $n$-kernel $\left(g^{0}, \ldots, g^{n-1}\right)$ of $g^{n}$, the following sequence is $n$-exact:

$$
X^{0} \stackrel{g^{0}}{\longrightarrow} X^{1} \stackrel{g^{1}}{\longrightarrow} \cdots \stackrel{g^{n-1}}{\longrightarrow} X^{n} \stackrel{g^{n}}{\longrightarrow} X^{n+1} .
$$

Let us give some important remarks regarding Definition 3.1 .

Remark 3.2. By Proposition 2.5 and Proposition 2.7 we can replace axiom (A2) by the following weaker version:

(A2') For every monomorphism $f^{0}: X^{0} \rightarrow X^{1}$ in $\mathcal{M}$ there exists an $n$-exact sequence:

$$
X^{0} \stackrel{f^{0}}{\longrightarrow} X^{1} \stackrel{f^{1}}{\longrightarrow} \cdots \stackrel{f^{n-1}}{\longrightarrow} X^{n} \stackrel{f^{n}}{\longrightarrow} X^{n+1} .
$$

Naturally, we can weaken axiom $\left(\mathrm{A} 2^{\mathrm{op}}\right)$ in a dual manner.

Remark 3.3. Let $\mathcal{M}$ be an $n$-abelian category. An immediate consequence of axioms (A1) and (A2) (resp. (A2 $\left.{ }^{\mathrm{op}}\right)$ is that every monomorphism (resp. epimorphism) in $\mathcal{N}$ appears as the leftmost (resp. rightmost) morphism in some $n$-exact sequence.

Remark 3.4. Let $m$ and $n$ be distinct positive integers. Note that the only categories which are both $n$-abelian and $m$-abelian are the semisimple categories, see Corollary 3.10

Note that 1-abelian categories are precisely abelian categories in the usual sense. It is easy to see that abelian categories are idempotent complete; thus, if $n=1$, then axiom (A0) in Definition 3.1 is redundant. However, if $n \geq 2$, then axiom $(\mathrm{A} 0)$ is independent from the remaining axioms as shown by the following example. 
Example 3.5. Let $n \geq 2$ and $K$ be a field. Consider the full subcategory $\mathcal{V}$ of $\bmod K$ given by the finite dimensional $K$-vector spaces of dimension different from 1. Then, $\mathcal{V}$ is not idempotent complete but it satisfies axioms (A1), (A2) and $\left(\mathrm{A} 2^{\mathrm{op}}\right)$

Proof. Firstly, note that $\mathcal{V}$ is an additive subcategory of $\bmod K$. The fact that $\mathcal{V}$ is not idempotent complete is obvious (consider the idempotent $0 \oplus 1_{K}: K^{2} \rightarrow K^{2}$ whose kernel is one-dimensional, for example). Let us show that $\mathcal{V}$ satisfies axiom (A1). Indeed, let $f: V \rightarrow W$ be a morphism in $V$. If coker $f$ has dimension different from 1 , then

$$
V \longrightarrow W \longrightarrow \operatorname{coker} f \longrightarrow 0 \longrightarrow \cdots \longrightarrow 0
$$

gives an $n$-cokernel of $f$ in $\mathcal{V}$. If coker $f$ has dimension 1 , then we can construct an $n$-cokernel of $f$ in $\mathcal{V}$ by a commutative diagram



where coker $f \rightarrow K^{3} \rightarrow K^{2}$ is a kernel-cokernel pair. We can construct an $n$-kernel of $f$ in a dual manner. This shows that $V$ satisfies axiom (A1), That $V$ satisfies axioms (A2) and (A2 $\left.{ }^{\mathrm{op}}\right)$ follows from Proposition 2.6 since contractible complexes with $n+2$ terms are in particular $n$-exact sequences by Proposition 2.5 .

Lemma 3.6. Let $\mathcal{C}$ be an idempotent complete additive category and suppose that we are given a sequence of morphisms in $\mathcal{C}$ of the form

$$
A \stackrel{f}{\longrightarrow} B \stackrel{g}{\longrightarrow} C \stackrel{h}{\longrightarrow} D
$$

If $g$ is a weak cokernel of $f$, and $h$ is both a split epimorphism and a cokernel of $g$, then $f$ admits a cokernel in $\mathcal{C}$.

Proof. Since $h$ is a split epimorphism there exists a morphism $i: D \rightarrow C$ such that $i h=1_{D}$. It follows that the morphism $e:=1_{C}-h i$ is idempotent. Since the category $\mathcal{C}$ is idempotent complete, there exists an object $E \in \mathcal{C}$ and morphisms $r: C \rightarrow E$ and $s: E \rightarrow C$ such that $s r=1_{E}$ and $r s=e$. Note that this implies that $r$ is an epimorphism and $s h=0$ for we have

$$
r(s h)=(1-h i) h=h-h=0 .
$$

We claim that $g r$ is a cokernel of $f$. Indeed, let $u: B \rightarrow B^{\prime}$ be a morphism such that $f u=0$. Since $g$ is a weak cokernel of $f$ there exists a morphism $v: C \rightarrow B^{\prime}$ such that $u=g v$. It follows that

$$
u=g v=g(1-h i) v=(g r)(s v) .
$$

This shows that $g r$ is a weak cokernel of $f$. It remains to show that $g r$ is an epimorphism. For this, let $w: E \rightarrow E^{\prime}$ be a morphism such that $(g r) w=0$. Since $h$ is a cokernel of $g$ there exists a morphism $x: D \rightarrow E^{\prime}$ such that $r w=h x$. It follows that

$$
w=(s r) w=s(h x)=0 .
$$

This shows that $g r$ is an epimorphism. Therefore $g r$ is a cokernel of $f$. 
Proposition 3.7. Let $\mathcal{M}$ be an additive category which satisfies axioms (A0) and (A1), and let $X$ a complex in $\mathrm{Ch}^{n-1}(\mathcal{C})$. If for all $1 \leq k \leq n-1$ the morphism $d^{k}$ is a weak cokernel of $d^{k-1}$, then $d^{n-1}$ admits a cokernel in $\mathcal{M}$.

Proof. If $n=1$, then the result follows trivially from axiom (A1). Hence we may assume that $n \geq 2$. By axiom (A1) there exists an $n$-cokernel

$$
\left(d^{k}: X^{k} \rightarrow X^{k+1} \mid n \leq k \leq 2 n-1\right)
$$

of $d^{n-1}$. Using axiom (A1) again together with the factorization property of weak cokernels we obtain a commutative diagram

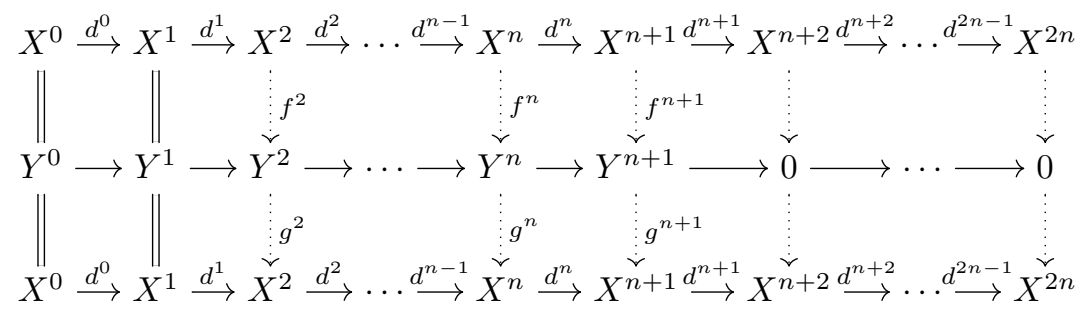

in which the middle row gives an $n$-cokernel of $d^{0}$. The Comparison Lemma 2.1 implies that there exists a morphism $h: X^{2 n} \rightarrow X^{2 n-1}$ such that $h d^{2 n-1}=1$. Hence we may apply Lemma 3.6 and reduce the length of the $n$-cokernel of $d^{n-1}$ by one morphism. Proceeding inductively, we deduce that $d^{n-1}$ has a cokernel in $\mathcal{M}$.

The importance of axiom (A0) becomes apparent in the following result, which asserts that $n$-abelian categories have $n$-pushout diagrams and $n$-pullback diagrams.

Theorem 3.8 (Existence of $n$-pushout diagrams). Let $\mathcal{M}$ be an additive category which satisfies axioms (A0) and (A1), Let $X$ be a complex in $\mathrm{Ch}^{n-1}(\mathrm{C})$, and a morphism $f: X^{0} \rightarrow Y^{0}$. Then, the following statements hold:

(i) Then, there exists an n-pushout diagram

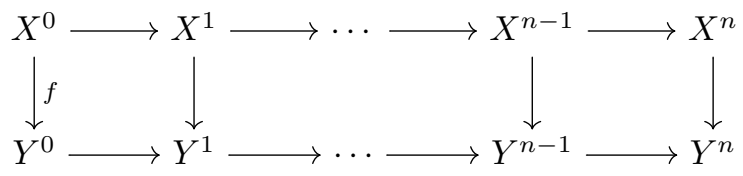

(ii) Suppose, moreover, that $\mathcal{M}$ is an n-abelian category. If $d_{X}^{0}$ is a monomorphism, then $d_{Y}^{0}$ is a monomorphism.

Proof. (i) We shall construct the complex $Y$ inductively. Set $f^{0}:=f$ and

$$
d_{C}^{-1}=\left[\begin{array}{c}
-d_{X}^{0} \\
f^{0}
\end{array}\right]: X^{0} \longrightarrow X^{1} \oplus Y^{0}
$$

Let $0 \leq k \leq n-2$ and suppose that for each $\ell \leq k$ we have constructed an object $Y^{\ell}$ and morphisms $f^{\ell}: X^{\ell} \rightarrow Y^{\ell}$ and $d_{Y}^{\ell-1}: Y^{\ell-1} \rightarrow Y^{\ell}$ such that $d_{C}^{\ell-2} d_{C}^{\ell-1}=0$ where

$$
d_{C}^{\ell-1}:=\left[\begin{array}{cc}
-d_{X}^{\ell} & 0 \\
f^{\ell} & d_{Y}^{\ell-1}
\end{array}\right]: X^{\ell} \oplus Y^{\ell-1} \longrightarrow X^{\ell+1} \oplus Y^{\ell}
$$


(compare with (2.4)). Then, by axiom (A1) the morphism $d_{C}^{k-1}$ has a weak cokernel $g^{k}:=\left[f^{k+1} d_{Y}^{k}\right]: X^{k+1} \oplus Y^{k} \rightarrow Y^{k+1}$. We claim that

$$
d_{C}^{k}:=\left[\begin{array}{cc}
-d_{X}^{k+1} & 0 \\
f^{k+1} & d_{Y}^{k}
\end{array}\right]: X^{k+1} \oplus Y^{k} \longrightarrow X^{k+2} \oplus Y^{k+1}
$$

is also a weak cokernel of $d_{C}^{k-1}$. Indeed, it is readily verified that $d_{C}^{k-1} d_{C}^{k}=0$. Let $u: X^{k+1} \oplus Y^{k} \rightarrow M$ be a morphism such that $d_{C}^{k-1} u=0$. Since $g^{k}$ is a weak cokernel of $d_{C}^{k-1}$, there exists a morphism $v: Y^{k+1} \rightarrow M$ such that $u=g^{k} v$. It follows that the following diagram is commutative:

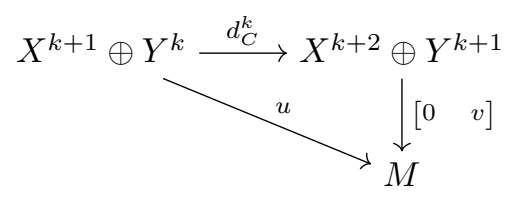

This shows that $d_{C}^{k}$ is a weak cokernel of $d_{C}^{k-1}$. Finally, Proposition 3.7 implies that the morphism $d_{C}^{n-2}$ admits a cokernel

$$
d_{C}^{n-1}: X^{n} \oplus Y^{n-1} \rightarrow Y^{n} .
$$

This shows that the tuple $\left(d_{C}^{0}, d_{C}^{1}, \ldots, d_{C}^{n-1}\right)$ is a weak cokernel of $d_{C}^{-1}$. The existence of the required commutative diagram follows from the fact that $C$ is a complex.

(iii) Finally, suppose that $\mathcal{N}$ is $n$-abelian and $d_{X}^{0}$ is a monomorphism. Note that this implies that $d_{C}^{-1}$ is also a monomorphism. Then, axiom (A2) implies that the $C$ is an $n$-exact sequence. In order to show that $d_{Y}^{0}$ is a monomorphism, let $u: M \rightarrow Y^{0}$ be a morphism such that $u d_{Y}^{0}=0$. It follows that the composition

$$
M \stackrel{\left[\begin{array}{l}
0 \\
u
\end{array}\right]}{\longrightarrow} X^{1} \oplus Y^{0} \stackrel{\left[\begin{array}{rr}
-d_{X}^{1} & 0 \\
f^{1} & d_{Y}^{0}
\end{array}\right]}{\longrightarrow} X^{2} \oplus Y^{1}
$$

vanishes. Given that $d_{C}^{-1}$ is a kernel of $d_{C}^{0}$, there exists a morphism $v: M \rightarrow X^{0}$ such that $v d_{X}^{0}=0$ and $v f^{0}=u$. Since $d_{X}^{0}$ is a monomorphism, we have $u=0$. This shows that $d_{Y}^{0}$ is a monomorphism.

We remind the reader that an additive category $\mathcal{C}$ is semisimple if every morphism $f: A \rightarrow B$ in $\mathcal{C}$ factors as $f=p i$, where $p$ is a split epimorphism and $i$ is a split monomorphism. The following result characterizes semisimple categories in terms of $n$-abelian categories.

Theorem 3.9. Let $\mathcal{C}$ be an additive category and $n$ a positive integer. Then, the $n$-abelian categories in which every n-exact sequence is contractible are precisely the semisimple categories.

Proof. Suppose that $\mathcal{C}$ is a semisimple category. We only show that $\mathcal{C}$ is idempotent complete. It is straightforward to verify that $\mathcal{C}$ satisfies the remaining axioms of $n$ abelian categories, the fact that every $n$-exact sequence in $\mathcal{C}$ is contractible follows immediately from Proposition 2.6. Let us show then that $\mathcal{C}$ is idempotent complete. Let $e: A \rightarrow A$ be an idempotent in $\mathcal{C}$. Since $\mathcal{C}$ is semisimple, $e$ factors as $e=p i$ where $p: A \rightarrow B$ is a split epimorphism and $i: B \rightarrow A$ is a split monomorphism. 
We claim that $i p=1_{B}$. Indeed, let $h: A \rightarrow B$ be a morphism such that $i h=1_{B}$. Given that $e^{2}=e$ we have

$$
p=p(i h)=e h=e^{2} h=(\text { pipi }) h=p(i p) .
$$

Since $p$ is an epimorphism we have $i p=1_{B}$ as claimed. This shows that $\mathcal{C}$ is idempotent complete.

Conversely, suppose that $\mathcal{C}$ is an $n$-abelian category in which every $n$-exact sequence is contractible and let $f: A \rightarrow B$ be a morphism in $\mathcal{C}$. We claim that $f$ admits both a kernel and a cokernel in $\mathcal{C}$. Indeed, by axiom (A1) there exists an $n$-cokernel $\left(f^{1}, \cdots, f^{n}\right)$ of $f$. By hypothesis, the epimorphism $f^{n}$ must split. Then, Lemma 3.6 implies that $f^{n-2}$ has a cokernel in $\mathcal{C}$. By applying this argument inductively we deduce that $f$ admits a cokernel in $\mathcal{C}$. By duality, $f$ also admits a kernel in $\mathcal{C}$. The remaining part of the proof is classical, compare for example with the proof of [18, Prop. 4.8].

We need to show that $f$ factors as $f=p i$ where $p$ is a split epimorphism and $i$ is a split monomorphism. Given that $f$ has both a kernel and a cokernel in $\mathcal{C}$, is easy to construct a commutative diagram



where $i$ is a kernel of $f$, and $p$ is a cokernel of $f$, and the sequences $K \rightarrow A \rightarrow J$ and $I \rightarrow B \rightarrow C$ are kernel-cokernel pairs. We claim that $g$ is an isomorphism, for which it is enough to show that is both a monomorphism and an epimorphism as all such morphisms split by hypothesis. By duality we only need to show that $g$ is an epimorphism.

Let $h: I \rightarrow I^{\prime}$ be a morphism such that $g h=0$. Firstly, by Theorem 3.8 there exists a commutative diagram

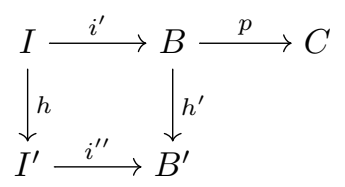

where $i^{\prime \prime}$ is a monomorphism. Secondly, we claim that $f h^{\prime}=0$. Indeed, we have

$$
f h^{\prime}=\left(p^{\prime} g i^{\prime}\right) h^{\prime}=p^{\prime}(g h) i^{\prime \prime}=0 .
$$

Therefore, since $p$ is a cokernel of $f$, there exists a morphism $j: C \rightarrow B^{\prime}$ such that $p j=h^{\prime}$. It follows that

$$
h i^{\prime \prime}=i^{\prime} h^{\prime}=i^{\prime}(p j)=0 .
$$

Finally, since $i^{\prime \prime}$ is a monomorphism, we have $h=0$. This shows that $g$ is an epimorphism.

Corollary 3.10. Let $m$ and $n$ be two distinct positive integers and $\mathcal{C}$ an additive category. If $\mathrm{C}$ is both $m$-abelian and $n$-abelian, then $\mathcal{C}$ is a semisimple category.

Proof. Without loss of generality we may assume that $m<n$. By Theorem 3.9 and Proposition 2.6 it is enough to show that every monomorphism in $\mathcal{C}$ splits. Let $f^{0}: X^{0} \rightarrow X^{1}$ be a monomorphism in $\mathcal{C}$ and let $\left(f^{1}, \ldots, f^{n}\right)$ be an $n$-cokernel of $f^{0}$, 
and $\left(g^{1}, \ldots, g^{m}\right)$ be an $m$-cokernel of $f^{0}$. It follows that there exists a commutative diagram

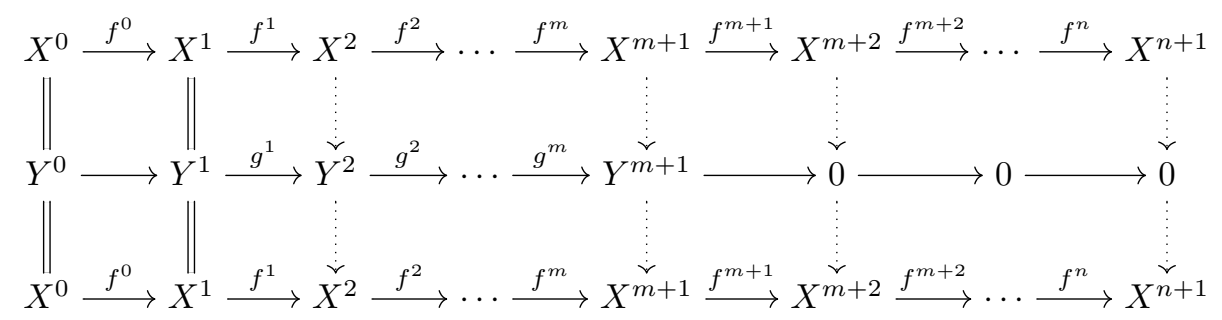

By the Comparison Lemma 2.1 there exists a morphism $h: X^{n+1} \rightarrow X^{n}$ such that $h f^{n}=1$. Thus $f^{n}$ is a split epimorphism. Then, as $\left(f^{0}, f^{1}, \ldots, f^{n}\right)$ is an $n$-exact sequence by axiom (A2) the dual of Proposition 2.6 implies that $f^{0}$ is a split monomorphism.

3.2. Projective objects in $n$-abelian categories. We remind the reader of the following classical definition.

Definition 3.11. Let $\mathcal{C}$ be an additive category. We say that $P \in \mathcal{C}$ is projective if for every epimorphism $f: A \rightarrow B$ the sequence of abelian groups

$$
\mathcal{C}(P, A) \stackrel{? \cdot f}{\longrightarrow} \mathcal{C}(P, B) \longrightarrow 0
$$

is exact. The concept of injective object in $\mathcal{C}$ is defined dually.

Our aim is to prove the following important property of projective objects in an $n$-abelian category.

Theorem 3.12. Let $\mathcal{M}$ be an n-abelian category and $P \in \mathcal{M}$ a projective object. Then, for every morphism $f: L \rightarrow M$ and every weak cokernel $g: M \rightarrow N$ of $f$, the sequence of abelian groups

$$
\mathcal{M}(P, L) \stackrel{? \cdot f}{\longrightarrow} \mathcal{M}(P, M) \stackrel{? \cdot g}{\longrightarrow} \mathcal{M}(P, N)
$$

is exact.

For the proof of Theorem 3.12 we need the following result which, albeit technical, is interesting in its own right.

Proposition 3.13. Let $\mathcal{M}$ be an n-abelian category, $f^{0}: X^{0} \rightarrow X^{1}$ a morphism in $\mathcal{M}$ and $\left(f^{k}: X^{k} \rightarrow X^{k+1} \mid 1 \leq k \leq n\right)$ an n-cokernel of $f^{0}$. Then, for every $k \in\{0,1, \ldots, n\}$ and every $\ell \in\{1, \ldots, n\}$ there exists morphisms $g_{k}^{\ell}: Y_{k}^{\ell} \rightarrow Y_{k}^{\ell-1}$ (with $Y_{k}^{0}:=X^{k}$ ) and $p_{k}^{\ell-1}: Y_{k}^{\ell-1} \rightarrow Y_{k+1}^{\ell}$ satisfying the following properties:

(i) For every $k \in\{0,1, \ldots, n\}$ the diagram



commutes.

(ii) The sequence $\left(g_{k}^{n}, \ldots, g_{k}^{1}\right)$ is an n-kernel of $f^{k}$. 
(iii) The diagram

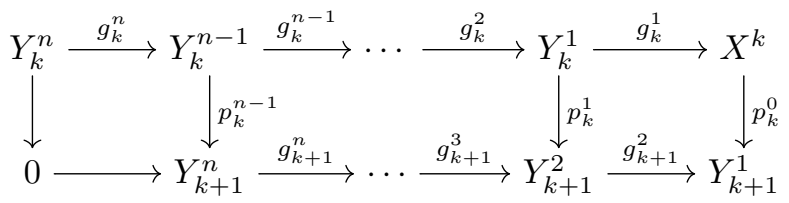

is both an n-pullback diagram and a good n-pushout diagram, see DefinitionProposition 2.14. In particular, the morphism

$$
\left[\begin{array}{ll}
p_{k}^{0} & g_{k+1}^{2}
\end{array}\right]: X^{k} \oplus Y_{k+1}^{2} \longrightarrow Y_{k+1}^{1}
$$

is an epimorphism.

(iv) If $k \neq 0$, then the sequence $\left(g_{k}^{k-1}, \ldots, g_{k}^{1}, f^{k}, \ldots, f^{n}\right)$ is an $n$-cokernel of the morphism $g_{k}^{k}$.

Proof. We proceed by induction on $k$, beginning with the case $1 \neq k=n$; the case $n=1$ of $n$-abelian categories is straightforward. By axiom (A1) there exists an $n$-kernel $\left(g_{n}^{n}, \ldots, g_{1}^{n}\right)$ of $f^{n}$ and by axiom $\left(\mathrm{A} 2^{\mathrm{op}}\right)$ the sequence $\left(g_{n}^{n}, \ldots, g_{1}^{1}, f^{n}\right)$ is an $n$-exact sequence. Note that this implies that the diagram

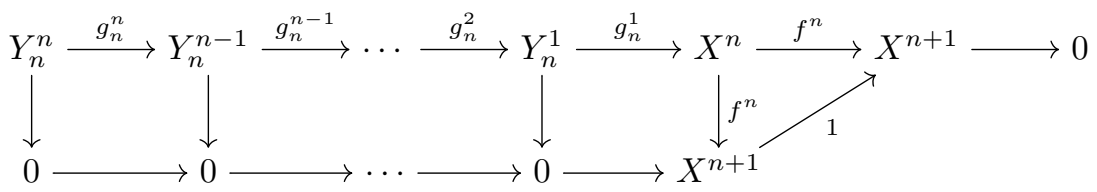

is a both an $n$-pullback diagram and an $n$-pushout diagram. By Definition-Proposition 2.14 we can replace this diagram by a good $n$-pushout diagram

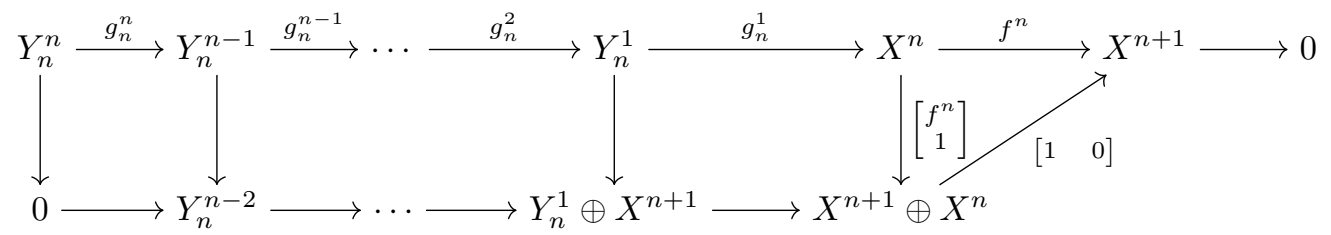

Note that this passage does not change the fact that the top row gives an $n$-kernel of $f^{n}$. This shows that the result is holds in this case.

Let $2 \leq k \leq n$ and suppose that we have constructed a commutative diagram of the form (3.2) with the required properties. Since $f^{k-1} f^{k}=0$ and $g_{k}^{1}$ is a weak kernel of $f^{k}$ there exists a morphism $p_{k-1}^{0}: X^{k-1} \rightarrow Y_{k}^{1}$ such that $f^{k-1}=p_{k-1}^{0} g_{k}^{1}$. We claim that the morphism

$$
\left[\begin{array}{ll}
p_{k-1}^{0} & g_{k}^{2}
\end{array}\right]: X^{k-1} \oplus Y_{k}^{2} \longrightarrow Y_{k}^{1}
$$

is an epimorphism. Indeed, let $u: Y_{k}^{1} \rightarrow M$ be a morphism such that $p_{k-1}^{0} u=0$ and $g_{k}^{2} u=0$. Given that $g_{k}^{1}$ is a weak cokernel of $g_{k}^{2}$ there exists a morphism $v: X^{k} \rightarrow M$ such that $u=g_{k}^{1} v$. It follows that

$$
f^{k-1} v=\left(p_{k-1}^{0} g_{k}^{1}\right) v=p_{k-1}^{0} u=0 .
$$


Then, since $f^{k}$ is a weak cokernel of $f^{k-1}$, there exists a morphism $w: X^{k+1} \rightarrow M$ such that $v=f^{k} w$. Thus, we have

$$
u=g_{k}^{1} v=g_{k}^{1}\left(f^{k} w\right)=0
$$

The claim follows. By Theorem 3.8 there exists an $n$-pullback diagram

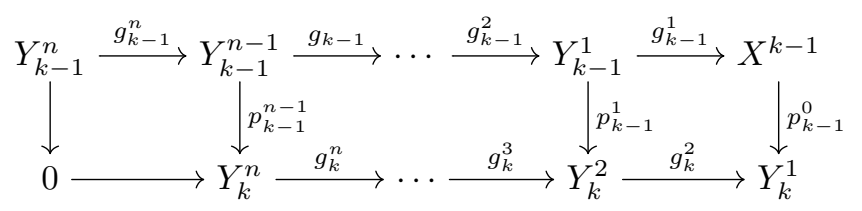

Note that axiom $\left(\mathrm{A} 2^{\mathrm{op}}\right)$ implies that it this diagram is also an $n$-pushout diagram, and that Proposition 2.12 implies that it has the required properties, except that it need not be a good $n$-pushout diagram. Using Definition-Proposition 2.14 we may replace this diagram by a good $n$-pushout diagram. Note that since the passage to a good $n$-pushout diagram amounts to adding a contractible $n$-exact sequence it does not alter the properties of the previously constructed diagrams.

Finally, let $k=1$. We have a commutative diagram

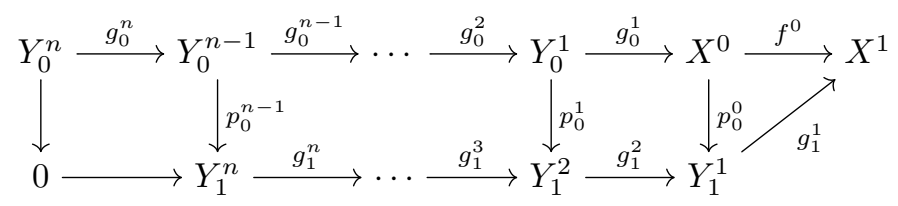

where the leftmost $n$ squares form an $n$-pullback diagram; we claim that they form moreover an $n$-pushout diagram. To show this, by axiom $\left(\mathrm{A} 2^{\mathrm{op}}\right)$ it is enough to show that the morphism

$$
\left[p_{0}^{0} \quad g_{1}^{2}\right]: X^{0} \oplus Y_{1}^{2} \longrightarrow Y_{1}^{1}
$$

is an epimorphism. Let $u: Y_{1}^{1} \rightarrow M$ be a morphism such that $p_{0}^{0} u=0$ and $g_{1}^{2}=$ 0 . Put $Y_{0}^{0}:=X^{0}$ and $u^{0}:=u$. Proceeding inductively, since the diagrams we constructed are good $n$-pushout diagrams, for each $k \in\{1, \ldots, n-1\}$ we obtain a commutative diagram

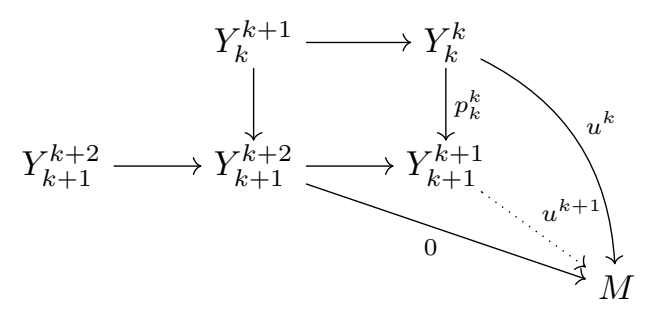

Moreover, by Theorem 3.8 there exists a commutative diagram

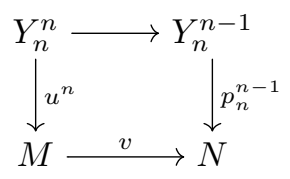


where $v$ is a monomorphism. It readily follows that

$$
u=p_{1}^{1} \cdots p_{n-1}^{n-1} u^{n} \quad \text { and } \quad u v=g_{1}^{1} p_{1}^{0} \cdots p_{n}^{n-1} .
$$

Next, observe that

$$
f^{0}\left(p_{1}^{0} \cdots p_{n}^{n-1}\right)=p_{0}^{0} g_{1}^{1} p_{1}^{0} \cdots p_{n}^{n-1}=p_{0}^{0} u v=0 .
$$

Given that $f^{1}$ is a weak cokernel of $f^{0}$, there exists a morphism $w: X^{1} \rightarrow N$ such that $f^{1} w=p_{1}^{0} \cdots p_{n}^{n-1}$. Finally, we have

$$
u v=g_{1}^{1}\left(p_{1}^{0} \cdots p_{n}^{n-1}\right)=g_{1}^{1} f^{1} w=0 .
$$

Since $v$ is a monomorphism, we have $u=0$ which is what we needed to show.

We are ready to give the proof of Theorem 3.12

Proof of Theorem 3.12, By Proposition 3.13 there exists a commutative diagram



where $i$ is a weak kernel of $g$, we have $q i=0$, and

$$
[p \quad q]: L \oplus K_{2} \longrightarrow K_{1}
$$

is an epimorphism.

Let $h: P \rightarrow M$ be a morphism such that $h g=0$. Since $i$ is a weak kernel of $g$, there exists a morphism $j: P \rightarrow K_{1}$ such that $h=j i$. Given that $P$ is projective and $L \oplus K_{2} \rightarrow K_{1}$ is an epimorphism, there exist morphisms $j^{\prime}: P \rightarrow L$ and $j^{\prime \prime}: P \rightarrow K_{2}$ such that $j=j^{\prime} p+j^{\prime \prime} q$. It follows that

$$
h=j i=\left(j^{\prime} p+j^{\prime \prime} q\right) i=j^{\prime} p i=j^{\prime} f .
$$

Hence $h$ factors through $f$. This shows that the sequence (3.1) is exact.

3.3. $n$-abelian categories and cluster-tilting. Recall that a subcategory $\mathcal{C}$ of an abelian category $\mathcal{A}$ is cogenerating if for every object $X \in \mathcal{A}$ there exists an object $Y \in \mathcal{C}$ and a monomorphism $X \rightarrow Y$. The concept of generating subcategory is defined dually. We use the following variant of the definition of a cluster-tilting subcategory of an abelian category.

Definition 3.14. Let $\mathcal{A}$ be an abelian category and $\mathcal{M}$ a generating-cogenerating subcategory of $\mathcal{A}$. We say that $\mathcal{M}$ is an $n$-cluster-tilting subcategory of $\mathcal{A}$ if $\mathcal{M}$ is functorially finite (see Subsection 2.1) in $\mathcal{A}$ and

$$
\begin{aligned}
\mathcal{M} & =\left\{X \in \mathcal{A} \mid \forall i \in\{1, \ldots, n-1\} \quad \operatorname{Ext}_{\mathcal{A}}^{i}(X, \mathcal{M})=0\right\} \\
& =\left\{X \in \mathcal{A} \mid \forall i \in\{1, \ldots, n-1\} \quad \operatorname{Ext}_{\mathcal{A}}^{i}(\mathcal{M}, X)=0\right\} .
\end{aligned}
$$

Note that $\mathcal{A}$ itself is the unique 1-cluster-tilting subcategory of $\mathcal{A}$.

Remark 3.15. Let $\mathcal{A}$ be an abelian category and $\mathcal{M}$ an $n$-cluster-tilting subcategory of $\mathcal{A}$. Since $\mathcal{M}$ is a cogenerating subcategory of $\mathcal{A}$, for all $A \in \mathcal{A}$ each left $\mathcal{M}$-approximation of $A$ is a monomorphism.

The following result provides us with a way of recognizing $n$-abelian categories. 
Theorem 3.16. Let $\mathcal{A}$ be an abelian category and $\mathcal{N}$ an n-cluster-tilting subcategory of $\mathcal{A}$. Then, $\mathcal{M}$ is an n-abelian category.

To prove Theorem 3.16 we need the following technical results.

Proposition 3.17. Let $\mathcal{A}$ be an abelian category and $\mathcal{M}$ an $n$-cluster-tilting subcategory of $\mathcal{A}$. Then, for all $A \in \mathcal{A}$ there exists an exact sequence

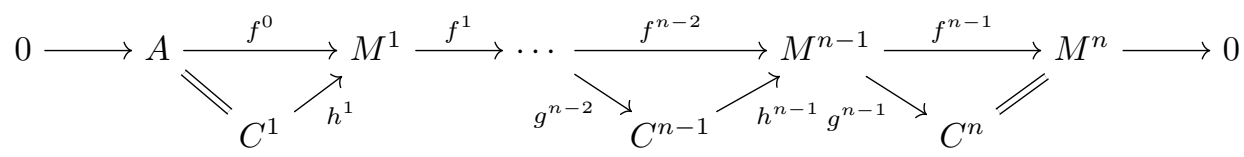

satisfying the following properties:

(i) For each $k \in\{1, \ldots, n\}$ we have $M^{k} \in \mathcal{M}$.

(ii) For each $k \in\{1, \ldots, n-1\}$ the morphism $h^{k}: C^{k} \rightarrow M^{k}$ is a left $\mathcal{M}$ approximation.

(iii) For each $k \in\{1, \ldots, n-1\}$ the morphism $g^{k}: M^{k} \rightarrow C^{k+1}$ is a cokernel of $h^{k}: C^{k} \rightarrow M^{k}$.

(iv) For all $M \in \mathcal{M}$ the induced sequence of abelian groups

$$
0 \longrightarrow \mathcal{A}\left(M^{n}, M\right) \longrightarrow \cdots \longrightarrow \mathcal{A}\left(M^{1}, M\right) \longrightarrow \mathcal{A}(A, M) \longrightarrow 0
$$

is exact.

Proof. This proof is an adaptation of the proof of [30, Thm. 2.2.3]. Given that for all $k \in\{1, \ldots, n-1\}$ the morphism $h^{k}: C^{k} \rightarrow M^{k}$ is a left $\mathcal{M}$-approximation, it readily follows that the sequence

$$
0 \longrightarrow \mathcal{A}\left(M^{n}, M\right) \longrightarrow \cdots \longrightarrow \mathcal{A}\left(M^{1}, M\right) \longrightarrow \mathcal{A}(A, M) \longrightarrow 0
$$

is exact. It remains to show that $C^{n} \in \mathcal{M}$.

We claim that for each $M \in \mathcal{M}$ and each $k \in\{2, \ldots, n\}$ we have $\operatorname{Ext}_{\mathcal{A}}^{i}\left(C^{k}, M\right)=$ 0 for all $1 \leq i \leq k-1$. We proceed by induction on $k$. First, note that for all $M \in \mathcal{M}$ applying the contravariant functor $\mathcal{A}(-, M)$ to the exact sequence $0 \rightarrow A \stackrel{f^{0}}{\longrightarrow} M^{2} \rightarrow C^{2}$ we have an exact sequence

$$
\mathcal{A}\left(M^{1}, M\right) \stackrel{f^{0} \cdot ?}{\longrightarrow} \mathcal{A}(A, M) \longrightarrow \operatorname{Ext}_{\mathcal{A}}^{1}\left(C^{2}, M\right) \longrightarrow \operatorname{Ext}_{\mathcal{A}}^{1}\left(M^{1}, M\right)=0 .
$$

Moreover, the morphism $\mathcal{A}\left(M^{1}, M\right) \rightarrow \mathcal{A}(A, M)$ is an epimorphism for $f^{0}$ is a left $\mathcal{M}$-approximation of $\mathcal{A}$. Thus we have $\operatorname{Ext}_{\mathcal{A}}^{1}\left(C^{2}, M\right)=0$ as required.

Let $2 \leq k \leq n-1$ and suppose that the claim holds for all $\ell \leq k$. Note that since $\mathcal{M}$ is a cogenerating subcategory of $\mathcal{A}$, the morphism $h^{k}$ is a monomorphism. In particular, we have that $h^{k}$ is a kernel of $g^{k}$. For all $M \in \mathcal{M}$ and for each $2 \leq i \leq k$, applying the contravariant functor $\mathcal{A}(-, M)$ to the exact sequence $0 \rightarrow C^{k} \rightarrow M^{k} \rightarrow C^{k+1} \rightarrow 0$ yields an exact sequence of the form

$$
0=\operatorname{Ext}_{\mathcal{A}}^{i-1}\left(C^{k}, M\right) \longrightarrow \operatorname{Ext}_{\mathcal{A}}^{i}\left(C^{k+1}, M\right) \longrightarrow \operatorname{Ext}_{\mathcal{A}}^{i}\left(M^{k}, M\right)=0
$$

Therefore $\operatorname{Ext}_{\mathcal{A}}^{i}\left(C^{k+1}, M\right)=0$ for all $2 \leq i \leq k$. Moreover, since $h^{k}$ is a left $\mathcal{M}$-approximation of $Y^{k}$, the induced morphism $\mathcal{A}\left(M^{k}, M\right) \rightarrow \mathcal{A}\left(C^{k}, M\right)$ is an 
epimorphism. Hence, applying the contravariant functor $\mathcal{A}(-, M)$ to the exact sequence $0 \rightarrow C^{k} \rightarrow M^{k} \rightarrow C^{k+1} \rightarrow 0$ yields an exact sequence

$$
\mathcal{A}\left(M^{k}, M\right) \longrightarrow \mathcal{A}\left(C^{k}, M\right) \longrightarrow \operatorname{Ext}_{\mathcal{A}}^{1}\left(C^{k+1}, M\right) \longrightarrow \operatorname{Ext}_{\mathcal{A}}^{1}\left(M^{k}, M\right)=0
$$

where the left morphism is an epimorphism. Thus $\operatorname{Ext}_{\mathcal{A}}^{1}\left(C^{k+1}, M\right)=0$. This finishes the induction step. We have shown that for all $M \in \mathcal{M}$ we have $\operatorname{Ext}_{\mathcal{A}}^{i}\left(C^{n}, M\right)=$ 0 for all $i \in\{1, \ldots, n-1\}$. Since $\mathcal{M}$ is an $n$-cluster-tilting subcategory of $\mathcal{M}$, this implies $C^{n}=M^{n} \in \mathcal{M}$ as required.

Proposition 3.18. Let $\mathcal{A}$ be an abelian category, $B \in \mathcal{A}$, and $\mathcal{M}$ a subcategory of $\mathcal{A}$ such that $\operatorname{Ext}_{\mathcal{A}}^{k}(\mathcal{M}, B)=0$ for all $k \in\{0,1, \ldots, n-1\}$. Consider an exact sequence

$$
M_{n} \longrightarrow M_{n-1} \longrightarrow \cdots \longrightarrow M_{1} \longrightarrow M_{0} \longrightarrow A \longrightarrow 0
$$

in $\mathcal{A}$ such that $M_{k} \in \mathcal{M}$ for all $k \in\{0,1, \ldots, n-1\}$. Then, for each $k \in$ $\{1, \ldots, n-1\}$ there is an isomorphism between $\operatorname{Ext}_{\mathcal{A}}^{k}(A, B)$ and the cohomology of the induced complex

$$
\mathcal{A}\left(M_{0}, B\right) \longrightarrow \mathcal{A}\left(M_{1}, B\right) \longrightarrow \cdots \longrightarrow \mathcal{A}\left(M_{n-1}, B\right) \longrightarrow \mathcal{A}\left(M_{n}, B\right)
$$

at $\mathcal{A}\left(M_{k}, B\right)$.

Proof. Let $A_{k}:=\operatorname{coker}\left(M_{k+1} \rightarrow M_{k}\right)$. Note that $A_{0}=A$. Firstly, let us show that for each $k \in\{1, \ldots, n-1\}$ (the case $k=0$ being clear) there exist isomorphisms

$$
\operatorname{Ext}_{\mathcal{A}}^{k}\left(A_{0}, B\right) \cong \operatorname{Ext}_{\mathcal{A}}^{k-1}\left(A_{1}, B\right) \cong \cdots \cong \operatorname{Ext}_{\mathcal{A}}^{1}\left(A_{k-1}, B\right) \text {. }
$$

The case $k=1$ is obvious. If $2 \leq k \leq n-1$, then for each $2 \leq \ell \leq k$ applying the functor $\mathcal{A}(-, B)$ to the exact sequence $0 \rightarrow A_{k-\ell+1} \rightarrow M_{k-\ell} \rightarrow A_{k-\ell} \rightarrow 0$ yields an exact sequence

$$
0={ }_{\mathcal{A}}^{\ell-1}\left(M_{k-\ell}, B\right) \rightarrow{ }_{\mathcal{A}}^{\ell-1}\left(A_{k-\ell+1}, B\right) \rightarrow{ }_{\mathcal{A}}^{\ell}\left(A_{k-\ell}, B\right) \rightarrow_{\mathcal{A}}^{\ell}\left(M_{k-\ell}, B\right)=0
$$

where we omitted $\operatorname{Ext}_{\mathcal{A}}$ because of lack of space. The claim follows.

Secondly, let us show that $\operatorname{Ext}_{\mathcal{A}}^{1}\left(A_{k-1}, B\right)$ is isomorphic to the cohomology of the complex $(3.3)$ at $\mathcal{A}\left(M_{k}, B\right)$. This follows by definition from the commutative diagram

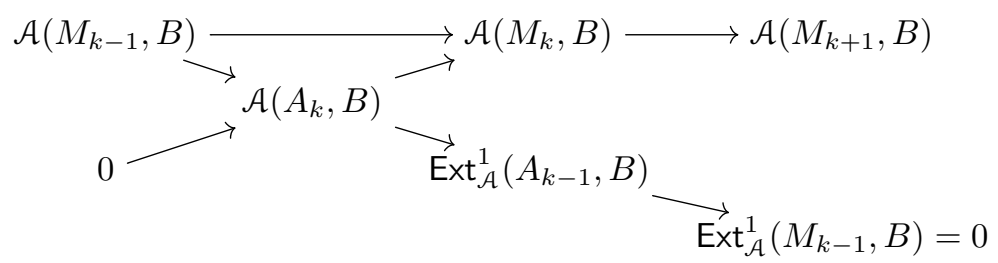

which is the glueing of two exact sequences. This concludes the proof.

Now we give the proof of Theorem 3.16 .

Proof of Theorem 3.16. We shall show that $\mathcal{M}$ satisfies the axioms of Definition 3.1.

(A0) Since the abelian category $\mathcal{A}$ is idempotent complete, it follows immediately from the definition of $n$-cluster-tilting subcategory that $\mathcal{M}$ also is idempotent complete. 
(A1) Let $f: A \rightarrow B$ be a morphism in $\mathcal{M}$. Let $B \rightarrow C$ be a cokernel of $f$, applying Proposition 3.17 to $C$ gives the desired $n$-cokernel of $f$. By duality, $f$ has an $n$-cokernel.

(A2) Let $f^{0}: X^{0} \rightarrow X^{1}$ be a monomorphism in $\mathcal{A}$ such that $X^{0}, X^{1} \in \mathcal{M}$ and let $\left(f^{k}: X^{k} \rightarrow X^{k+1} \mid 1 \leq k \leq n\right)$ be an $n$-cokernel of $f^{0}$ in $\mathcal{M}$ obtained as in the previous paragraph (we remind the reader of Remark 3.2). Applying the dual of Proposition 3.18 to the exact sequence

$$
0 \longrightarrow X^{0} \stackrel{f^{0}}{\longrightarrow} X^{1} \stackrel{f^{1}}{\longrightarrow} \cdots \stackrel{f^{n-1}}{\longrightarrow} X^{n} \stackrel{f^{n}}{\longrightarrow} X^{n+1},
$$

we obtain that for all $Y \in \mathcal{M}$ and for all $k \in\{1, \ldots, n-1\}$ the cohomology of the induced complex

$$
\mathcal{A}\left(Y, X^{1}\right) \longrightarrow \cdots \longrightarrow \mathcal{A}\left(Y, X^{n}\right) \longrightarrow \mathcal{A}\left(Y, X^{n+1}\right)
$$

at $\mathcal{A}\left(Y, X^{k+1}\right)$ is isomorphic to $\operatorname{Ext}_{\mathcal{A}}^{k}\left(Y, X^{0}\right)$ which vanishes since $\mathcal{M}$ is an $n$-clustertilting subcategory of $\mathcal{A}$. This shows that $\left(f^{0}, \ldots, f^{n-1}\right)$ is an $n$-kernel of $f^{n}$ in $\mathcal{M}$. That $\mathcal{M}$ also satisfies axiom $\left(\mathrm{A} 2^{\mathrm{op}}\right)$ now follows by duality. This concludes the proof of the theorem.

Definition 3.19. Let $\mathcal{M}$ be an $n$-abelian category. We say that $\mathcal{M}$ is projectively generated if for every object $M \in \mathcal{M}$ there exists a projective object $P \in \mathcal{M}$ and an epimorphism $P \rightarrow M$. The notion of injectively cogenerated $n$-abelian category is defined dually.

Our next aim is to show that a partial converse of Theorem 3.16 holds for projectively generated $n$-abelian categories. For this, we remind the reader of the notion of coherent modules on an additive category.

Let $\mathcal{C}$ be a small additive category. A $\mathcal{C}$-module is a contravariant functor $F: \mathcal{C} \rightarrow$ $\operatorname{Mod} \mathbb{Z}$. The category Mod $\mathcal{C}$ of $\mathcal{C}$-modules is an abelian category. Morphisms in Mod $\mathcal{C}$ are natural transformations of contravariant functors. If $M, N \in \operatorname{Mod} \mathcal{C}$, then we denote the set of natural transformations $M \rightarrow N$ by $\operatorname{Hom}_{\mathcal{C}}(M, N)$. As a consequence of Yoneda's lemma, representable functors are projective objects in Mod $\mathcal{C}$. The category of coherent $\mathcal{C}$-modules, denoted by $\bmod \mathcal{C}$, is the full subcategory of Mod $\mathcal{C}$ whose objects are the $\mathcal{C}$-modules $F$ such that there exists a morphism $f: X \rightarrow Y$ in $\mathcal{C}$ and an exact sequence of functors

$$
\mathcal{C}(-, X) \stackrel{? \cdot f}{\longrightarrow} \mathcal{C}(-, Y) \longrightarrow F \longrightarrow 0 .
$$

Note that mod $\mathcal{C}$ is closed under cokernels and extensions in Mod $\mathcal{C}$. Moreover, $\bmod \mathcal{C}$ is closed under kernels in Mod $\mathcal{C}$ if and only if $\mathcal{C}$ has weak kernels, in which case $\bmod \mathcal{C}$ is an abelian category, see [4, page 41]. For further information on coherent $\mathcal{C}$-modules we refer the reader to 3 .

Our aim is to prove the following theorem.

Theorem 3.20. Let $\mathcal{N}$ be a small projectively generated $n$-abelian category. Let $\mathcal{P}$ be the category of projective objects in $\mathcal{M}$ and $F: \mathcal{M} \rightarrow \bmod \mathcal{P}$ the functor defined by $F X:=\left.\mathcal{M}(-, X)\right|_{\mathcal{P}}$. Also, let

$$
F \mathcal{M}:=\{M \in \bmod \mathcal{P} \mid \exists X \in \mathcal{M} \text { such that } M \cong F X\}
$$


be the essential image of $F$. If there exist an exact duality $D: \bmod \mathcal{P} \rightarrow \bmod \mathcal{P}^{\text {op }}$,


of $\bmod \mathcal{P}$.

Remark 3.21. In Theorem 3.20 , the condition on existence of an exact duality $D: \bmod \mathcal{P} \rightarrow \bmod \mathcal{P}^{o p}$ is satisfied, for example, if $\mathcal{P}$ is a dualizing $k$-variety over a commutative artinian ring in the sense of [5].

In fact, instead of proving Theorem 3.20, we prove the following more general statement.

Lemma 3.22. Let $\mathcal{M}$ be a small projectively generated $n$-abelian category. Let $\mathcal{P}$ be the category of projective objects in $\mathcal{M}$ and $F: \mathcal{M} \rightarrow \bmod \mathcal{P}$ the functor defined by $F X:=\left.\mathcal{M}(-, X)\right|_{\mathcal{P}}$. Also, let

$$
F \mathcal{M}:=\{M \in \bmod \mathcal{P} \mid \exists X \in \mathcal{M} \text { such that } M \cong F X\}
$$

be the essential image of $F$. Then, the following statements hold:

(i) The category $\bmod \mathcal{P}$ is abelian.

(ii) The functor $F: \mathcal{M} \rightarrow \bmod \mathcal{P}$ is fully faithful.

(iii) For all $k \in\{1, \ldots, n-1\}$ we have $\operatorname{Ext}_{\mathcal{P}}^{k}(F \mathcal{M}, F \mathcal{M})=0$.

(iv) We have

$$
F \mathcal{M}=\left\{X \in \bmod \mathcal{P} \mid \forall k \in\{1, \ldots, n-1\} \operatorname{Ext}_{\mathcal{P}}^{k}(X, F \mathcal{N})=0\right\}
$$

where $\operatorname{Ext}_{\mathcal{P}}^{k}$ is the bifunctor of degree $k$ extensions in the abelian category $\bmod \mathcal{P}$.

(v) We have

$$
F \mathcal{M}=\left\{X \in \bmod \mathcal{P} \mid \forall k \in\{1, \ldots, n-1\} \operatorname{Ext}_{\mathcal{P}}^{k}(F \mathcal{M}, X)=0\right\} .
$$

(vi) The subcategory FM is contravariantly finite in $\bmod \mathcal{P}$.

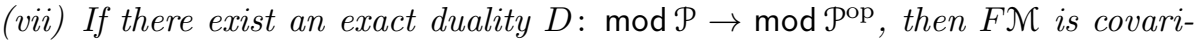
antly finite in $\bmod \mathcal{P}$. Hence, $F \mathcal{M}$ is a functorially finite subcategory of $\bmod \mathcal{P}$ in this case.

(viii) If there exist an exact duality $D: \bmod \mathcal{P} \rightarrow \bmod \mathcal{P}^{\text {op }}$, then $F \mathcal{M}$ is a generatingcogenerating subcategory of $\bmod \mathcal{P}$.

Proof. (ii) This statements follows from the fact that $\mathcal{M}$ has weak kernels 3 .

(ii) Let $M \in \mathcal{M}$. Since $\mathcal{M}$ is projectively generated, there exists an $n$-exact sequence

$$
K_{n} \longrightarrow \cdots \longrightarrow K_{1} \longrightarrow K_{0} \stackrel{u}{\longrightarrow} M
$$

where $K^{0}$ is a projective object. For the same reason, there exist a projective object $P^{1}$ and an epimorphism $v: P^{1} \rightarrow K_{1}$. Let $f=v u$ and put $P^{0}:=K_{0}$. It follows that the sequence

$$
F P^{1} \stackrel{F f}{\longrightarrow} F P^{0} \longrightarrow F M \longrightarrow 0
$$

is exact in $\operatorname{Mod} \mathcal{P}$. This shows that $F M \in \bmod \mathcal{P}$. That $F$ is fully faithful follows from Yoneda's lemma and the existence of a sequence of the form $F P^{1} \stackrel{F f}{\longrightarrow} F P^{0} \rightarrow$ $F M \rightarrow 0$ with $P^{0}, P^{1} \in \mathcal{M}$ for each $M \in \mathcal{M}$. 
(iii) Let us show that for every $M, N \in \mathcal{M}$ we have $\operatorname{Ext}_{\mathcal{P}}^{k}(F M, F N)=0$ for all $k \in\{1, \ldots, n-1\}$. Consider an $n$-exact sequence of the form 3.4. Applying $F$ to (3.4) yields an exact sequence

$$
0 \longrightarrow F K_{n} \rightarrow F K_{n-1} \longrightarrow \cdots \rightarrow F K_{1} \rightarrow F K_{0} \rightarrow F M \longrightarrow 0 .
$$

Since $F$ is fully faithful there is an isomorphism of complexes

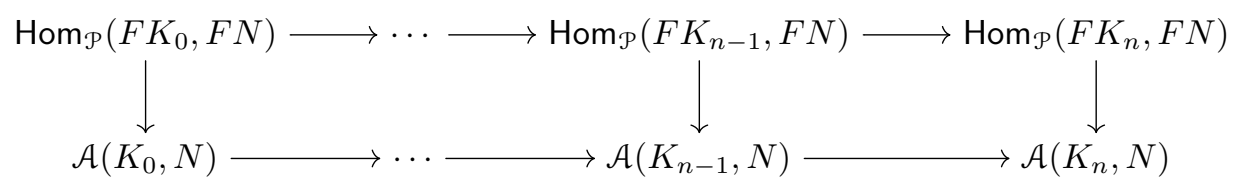

Note that the bottom row is exact by the property of $n$-exact sequences, hence the top row is also exact. Put $C_{\ell}:=\operatorname{coker}\left(F K_{\ell+1} \rightarrow F K_{\ell}\right)$. Note that $C_{0}:=F M$. There is an exact sequence

$$
\operatorname{Hom}_{\mathcal{P}}\left(F K_{0}, F N\right) \rightarrow \operatorname{Hom}_{\mathcal{P}}\left(C_{1}, F N\right) \rightarrow \operatorname{Ext}_{\mathcal{P}}^{1}(F M, F N) \rightarrow \operatorname{Ext}_{\mathcal{P}}^{1}\left(F K_{0}, F N\right)=0
$$

(recall that $F K_{0}$ is projective in $\bmod \mathcal{P}$ ). Since the top row of the above diagram is exact, this implies that $\operatorname{Ext}_{\mathcal{P}}^{1}(F M, F N)=0$. Hence we have $\operatorname{Ext}_{\mathcal{P}}^{1}(F \mathcal{M}, F \mathcal{M})=0$. We show that we have a sequence of isomorphisms

$$
\operatorname{Ext}_{\mathcal{P}}^{\ell}\left(C_{0}, F N\right) \cong \operatorname{Ext}_{\mathcal{P}}^{\ell-1}\left(C_{1}, F N\right) \cong \cdots \cong \operatorname{Ext}_{\mathcal{P}}^{1}\left(C_{\ell-1}, F N\right)=0
$$

for all $\ell \in\{1, \ldots, n-1\}$. If $\ell=1$, then the claim is trivial. Inductively, suppose that we have shown that $\operatorname{Ext}_{\mathcal{P}}^{m}(F \mathcal{N}, F \mathcal{M})=0$ for all $1 \leq m \leq \ell-1$. Firstly, note that applying the functor $\operatorname{Hom}_{\mathcal{P}}(-, F N)$ to the exact sequence $0 \rightarrow C_{\ell} \rightarrow$ $F K_{\ell-1} \rightarrow C_{\ell-1} \rightarrow 0$ gives an exact sequence

$$
\operatorname{Hom}_{\mathcal{P}}\left(F K_{\ell-1}, F N\right) \rightarrow \operatorname{Hom}_{\mathcal{P}}\left(C_{\ell}, F N\right) \rightarrow \operatorname{Ext}_{\mathcal{P}}^{1}\left(C_{\ell-1}, F N\right) \rightarrow \operatorname{Ext}_{\mathcal{P}}^{1}\left(F K_{\ell-1}, F N\right)=0,
$$

Since the top row of the above diagram is exact, this implies that $\operatorname{Ext}_{\mathcal{P}}^{1}\left(C_{\ell-1}, F N\right)=$ 0 . Secondly, by the induction hypothesis, for each $1 \leq m \leq \ell-1$ applying the functor $\operatorname{Hom}_{\mathcal{P}}(-, F N)$ to the exact sequence $0 \rightarrow C_{\ell-m} \rightarrow F K_{\ell-m-1} \rightarrow C_{\ell-m-1} \rightarrow$ 0 yields an exact sequence

$$
0={ }_{\mathcal{P}}^{m}\left(F K_{\ell-m-1}, F N\right) \rightarrow{ }_{\mathcal{P}}^{m}\left(C_{\ell-m}, F N\right) \rightarrow_{\mathcal{P}}^{m+1}\left(C_{\ell-m-1}, F N\right) \rightarrow_{\mathcal{P}}^{m+1}\left(F K_{\ell-m-1}, F N\right)=0
$$

where we omitted Ext because of lack of space (for $m=\ell-1$, recall that $F K_{0}$ is projective in $\bmod \mathcal{P})$. The claim follows since $C_{0}=F M$.

(iv) Let $G \in \bmod \mathcal{P}$ be such that for all $N \in \mathcal{M}$ and for all $k \in\{1, \ldots, n-1\}$ we have $\operatorname{Ext}_{\mathcal{P}}^{k}(G, F N)=0$. We need to show that there exists $M \in \mathcal{M}$ such that $G$ and $F M$ are isomorphic. For this, let

$$
F P_{1} \stackrel{F f_{0}}{\longrightarrow} F P_{0} \longrightarrow G \longrightarrow 0
$$

be a projective presentation of $G$ in $\bmod \mathcal{P}$ and let

$$
X_{n} \stackrel{f_{n}}{\longrightarrow} \cdots \longrightarrow X_{1} \stackrel{f_{1}}{\longrightarrow} X_{0}
$$


be an $n$-kernel of $f_{0}$ (by convention, $X_{0}:=P_{1}$ ). Let $N \in \mathcal{M}$. Applying the functor $\operatorname{Hom}_{\mathcal{P}}(F(-), F N)$ to the sequence $\left(f_{n}, \ldots, f_{1}, f_{0}\right)$ together with the fact that $F$ is fully faithful yields a commutative diagram

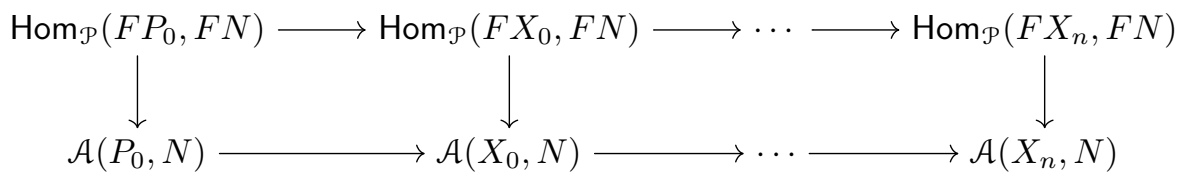

where the vertical arrows are isomorphisms. By what we showed in the previous paragraph and Proposition 3.18 for all $k \in\{1, \ldots, n-1\}$ the homology of the top row at $\operatorname{Hom}_{\mathcal{P}}\left(F X_{k-1}, F N\right)$ is isomorphic to $\operatorname{Ext}_{\mathcal{P}}^{k}(G, F N)$ which vanishes by hypothesis. It follows that the bottom row is an exact sequence. By applying Proposition 3.7 to $f_{n-1}$ and the sequence $\left(f_{n-2}, \ldots, f_{1}, f_{0}\right)$ we deduce that $f_{0}$ admits a cokernel $P_{0} \rightarrow M$ in $\mathcal{M}$. Finally, Theorem 3.12 implies that the sequence

$$
F P_{1} \stackrel{F f_{0}}{\longrightarrow} F P_{0} \longrightarrow F M \longrightarrow 0
$$

is exact in $\bmod \mathcal{P}$. Therefore $G$ is isomorphic to $F M$ which is what we needed to show.

(v) Let us show that if $G \in \bmod \mathcal{P}$ is such that for all $M \in \mathcal{M}$ and for all $k \in\{1, \ldots, n-1\}$ we have $\operatorname{Ext}_{\mathcal{P}}^{k}(F M, G)=0$, then $G \in F \mathcal{M}$. Indeed, let

$$
F P_{1} \stackrel{F f^{0}}{\longrightarrow} F P_{0} \longrightarrow G \longrightarrow 0
$$

be a projective presentation of $G$ in $\bmod \mathcal{P}$. By axiom (A1), there exists an $n$ cokernel $\left(f^{k}: M^{k-1} \rightarrow M^{k} \mid 1 \leq k \leq n\right)$ of $f^{0}$ in $\mathcal{M}$ (by convention, $M^{0}:=P^{0}$ ). Then, Theorem 3.12 implies that the sequence

$$
F P^{1} \stackrel{F f^{0}}{\longrightarrow} F P^{0} \stackrel{F f^{1}}{\longrightarrow} F M^{1} \stackrel{F f^{2}}{\longrightarrow} \cdots \stackrel{F f^{n}}{\longrightarrow} F M^{n} \longrightarrow 0
$$

is exact in $\bmod \mathcal{P}$. It follows that there is an exact sequence

$$
0 \longrightarrow G \longrightarrow F M^{1} \stackrel{F f^{2}}{\longrightarrow} \cdots \stackrel{F f^{n}}{\longrightarrow} F M^{n} \longrightarrow 0 .
$$

For each $k \in\{1, \ldots, n-1\}$ let $G^{k}:=\operatorname{ker} F f^{k+1}$; we claim that $G^{k} \in F \mathcal{M}$. Note that $\operatorname{Ext}_{\mathcal{P}}^{1}\left(F M^{k}, G^{k}\right)=0$ by hypothesis. In particular, $G^{n-1}$ is a direct summand of $F M^{n-1}$. Since $\mathcal{M}$ is idempotent complete, there exists an object $L \in \mathcal{M}$ such that $G^{n-1} \cong F L \in F \mathcal{M}$. Inductively, we deduce that $G^{1}=G \in F \mathcal{M}$.

(vi) Let $G \in \bmod \mathcal{P}$ and take a projective presentation

$$
F P^{1} \stackrel{F f}{\longrightarrow} F P^{0} \stackrel{p}{\longrightarrow} G \longrightarrow 0
$$

of $G$ in $\bmod \mathcal{P}$. Let $g: P^{0} \rightarrow M$ be a weak cokernel of $f$ in $\mathcal{M}$. We obtain the solid part of the following commutative diagram:

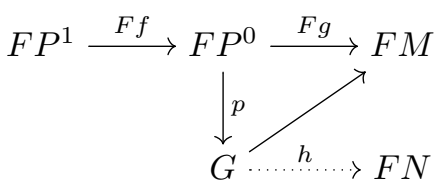


Let $h: G \rightarrow F N$ be a morphism in mod $\mathcal{P}$. Since $g$ is a weak cokernel there exists a morphism $F q: F M \rightarrow F N$ such that the diagram

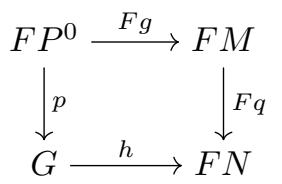

is commutative. Finally, given that $p$ is an epimorphism, we conclude that the diagram

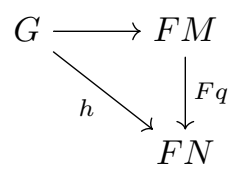

commutes. This shows that $G \rightarrow F M$ is a right $F \mathcal{N}$-approximation of $G$.

(vii) and (viii) It is clear that the assumptions imply that $\bmod \mathcal{P}$ is injectively cogenerated. From part (iii) we deduce that for every injective object $I \in \bmod \mathcal{P}$ we have $I \in F \mathcal{M}$. Hence (vii) follows by duality from part vii). Also, viii) follows since it is now clear that $\overline{F \mathcal{N}}$ is a cogenerating subcategory of mod $\mathcal{P}$.

\section{4. n-EXACT CATEGORIES}

In this section we introduce $n$-exact categories and establish their basic properties. We show that $n$-cluster-tilting subcategories of exact categories have a natural $n$-exact structure.

4.1. Definition and basic properties. The treatment of this section is parallel to Bühler's exposition of the basics of the theory of exact categories given in [18, Sec. 2].

Let $\mathcal{C}$ be an additive category. If $\mathcal{X}$ is a class of $n$-exact sequences in $\mathcal{C}$, then we call its members $\mathcal{X}$-admissible $n$-exact sequences. Furthermore, if

$$
X^{0} \succ d^{0} \longrightarrow X^{1} \stackrel{d^{1}}{\longrightarrow} \cdots \stackrel{d^{n-1}}{\longrightarrow} X^{n} \stackrel{d^{n}}{\longrightarrow} X^{n+1}
$$

is an $X$-admissible $n$-exact sequence, we say that $d^{0}$ is an $X$-admissible monomorphism and that $d^{n}$ is an $X$-admissible epimorphism. In analogy with [18, we depict $X$-admissible monomorphisms by $\longmapsto$ and $X$-admissible epimorphisms by $\rightarrow$. A sequence $\longrightarrow \rightarrow \cdots \rightarrow \rightarrow$ of $n+1$ morphisms always denotes an $\mathcal{X}$-admissible $n$-exact sequence. When the class $X$ is clear from the context, we write "admissible" instead of " $X$-admissible".

Definition 4.1. We say that a morphism $f: X \rightarrow Y$ of $n$-exact sequences in $\mathrm{Ch}^{n}(\mathrm{C})$ is a weak isomorphism if $f^{k}$ and $f^{k+1}$ are isomorphisms for some $k \in$ $\{0,1, \ldots, n+1\}$ with $n+2:=0$ (this terminology is borrowed from the theory of $(n+2)$-angulated categories, see Section 5.1). Note that weak isomorphisms induce isomorphisms in $\mathrm{H}(\mathrm{C})$ by Proposition 2.7

Definition 4.2. Let $n$ be a positive integer and $\mathcal{M}$ an additive category. An $n$ exact structure on $\mathcal{M}$ is a class $\mathcal{X}$ of $n$-exact sequences in $\mathcal{M}$, closed under weak isomorphisms of $n$-exact sequences, and which satisfies the following axioms:

(E0) The sequence $0 \longmapsto 0 \rightarrow \cdots \rightarrow 0 \rightarrow 0$ is an $\mathcal{X}$-admissible $n$-exact sequence. 
(E1) The class of $X$-admissible monomorphisms is closed under composition.

(E1 $\left.{ }^{\mathrm{op}}\right)$ The class of $\mathcal{X}$-admissible epimorphisms is closed under composition.

(E2) For each $X$-admissible $n$-exact sequence $X$ and each morphism $f: X^{0} \rightarrow$ $Y^{0}$, there exists an $n$-pushout diagram of $\left(d_{X}^{0}, \ldots, d_{X}^{n-1}\right)$ along $f$ such that $d_{Y}^{0}$ is an $\mathcal{X}$-admissible monomorphism. The situation is illustrated in the following commutative diagram:



(E2 $\left.{ }^{\text {op }}\right)$ For each $\mathcal{X}$-admissible $n$-exact sequence $X$ and each morphism $g: Y^{n+1} \rightarrow$ $X^{n+1}$, there exists an $n$-pullback diagram of $\left(d_{X}^{1}, \ldots, d_{X}^{n}\right)$ along $g$ such that $d_{Y}^{n}$ is an $X$-admissible epimorphism. The situation is illustrated in the following commutative diagram:

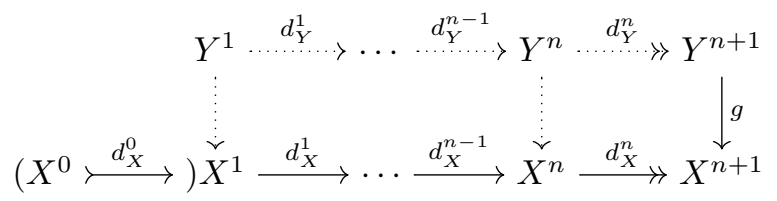

An $n$-exact category is a pair $(\mathcal{M}, \mathcal{X})$ where $\mathcal{M}$ is an additive category and $\mathcal{X}$ is an $n$-exact structure on $\mathcal{M}$. If the class $\mathcal{X}$ is clear from the context, we identify $\mathcal{M}$ with the pair $(\mathcal{M}, \mathcal{X})$.

Remark 4.3. Our choice of axioms for $n$-exact categories is inspired by Keller's minimal list of axioms for exact categories [36, App. A], although we opt for a more convenient self-dual collection. In particular, we point out to the reader who is more familiar with Quillen's axioms that the so-called "obscure axiom", axiom c) of [42, Sec. 2], is redundant, see [18, p. 4, Prop. 2.16].

The following result shows that $n$-abelian categories have a canonical $n$-exact structure. Therefore the class of $n$-exact categories contains the class of $n$-abelian categories.

Theorem 4.4. Let $\mathcal{M}$ be an $n$-abelian category and $\mathcal{X}$ the class of all $n$-exact sequences in $\mathcal{M}$. Then, $(\mathcal{M}, \mathcal{X})$ is an $n$-exact category.

Proof. We shall show that $(\mathcal{M}, \mathcal{X})$ satisfies the axioms of Definition 4.2 , It is obvious that the class $\mathcal{X}$ is closed under weak isomorphisms and that axiom (E0) is satisfied. By axioms (A1) and (A2) in Definition 3.1, every monomorphism in $\mathcal{M}$ is the leftmost morphism in an $n$-exact sequence, see Remark 3.3 . Since the composition of two monomorphisms is again a monomorphism, axiom (E1) is satisfied. That axiom $\left(\mathrm{E} 1^{\mathrm{op}}\right)$ is also satisfied then follows by duality. Finally, Theorem 3.8 and its dual show that axioms $(\mathrm{E} 2)$ and $\left(\mathrm{E} 2^{\mathrm{op}}\right)$ are satisfied. This shows that $(\mathcal{\mathcal { N }}, \mathcal{X})$ is an $n$-exact category.

We begin our investigation of the properties of $n$-exact categories with a simple but useful observation. 
Lemma 4.5. Let $(\mathcal{M}, X)$ be an $n$-exact category and $X^{0} \longmapsto X^{1}$ and admissible monomorphism. If the sequence $\left(X^{k} \rightarrow X^{k+1} \mid 1 \leq k \leq n\right)$ is an $n$-cokernel of $f^{0}$, then the sequence

$$
X: X^{0} \longleftrightarrow X^{1} \longrightarrow \cdots \longrightarrow X^{n} \longrightarrow X^{n+1}
$$

is an admissible $n$-exact sequence.

Proof. Since $X^{0} \longmapsto X^{1}$ is an admissible monomorphism, there exists an admissible $n$-exact sequence $Y$ whose first morphism is $X^{0} \longmapsto X^{1}$. By the factorization property of $n$-cokernels, there exists a weak isomorphism $X \rightarrow Y$. Then, $X \in X$ since the class $X$ is closed under weak isomorphisms.

The next result shows that the $n$-exact structure of an $n$-exact category is closed under direct sums.

Proposition 4.6. Let $(\mathcal{N}, X)$ be an n-exact category, and $X$ and $Y$ be admissible $n$-exact sequences. Then, their direct sum $X \oplus Y$ is an admissible $n$-exact sequence.

Proof. This is an adaptation of the proof of [18, Prop. 2.9]. Clearly, $X \oplus Y$ is an $n$-exact sequence. We claim that $d_{X}^{0} \oplus 1_{Y^{0}}$ is an admissible monomorphism. Indeed, the sequence

$$
X^{0} \oplus Y^{0} \stackrel{d_{X}^{0} \oplus 1_{Y 0}}{\longrightarrow} X^{1} \oplus Y^{0} \stackrel{\left(d_{X}^{1} 0\right)}{\longrightarrow} X^{2} \stackrel{d_{X}^{2}}{\longrightarrow} \cdots \stackrel{d_{X}^{n}}{\longrightarrow} X^{n+1}
$$

is an $n$-exact sequence. Since $d_{X}^{n}$ is an admissible epimorphism, it follows from the dual of Lemma 4.5 that this sequence is moreover an admissible $n$-exact sequence and therefore $d_{X}^{0} \oplus 1_{Y^{0}}$ is an admissible monomorphism. We can show that $1_{X^{1}} \oplus d_{Y}^{0}$ is an admissible monomorphism with a similar argument. Next, observe that

$$
d_{X}^{0} \oplus d_{Y}^{0}=\left(d_{X}^{0} \oplus 1_{Y^{0}}\right) \cdot\left(1_{X^{1}} \oplus d_{Y}^{0}\right) .
$$

By axiom (E1) we have that $d_{X}^{0} \oplus d_{Y}^{0}: X^{0} \oplus Y^{0} \rightarrow X^{1} \oplus Y^{1}$ is an admissible monomorphism. Since $X \oplus Y$ is an admissible $n$-exact sequence, the claim follows from Lemma 4.5.

Remark 4.7. Let $(\mathcal{M}, X)$ be an $n$-exact category with $n \geq 2$. It follows from (E0) and the fact that $X$ is assumed to be closed under weak isomorphisms that that for all $X \in \mathcal{M}$ the morphism $1_{X}$ is an admissible monomorphism. Indeed, there is a weak isomorphism

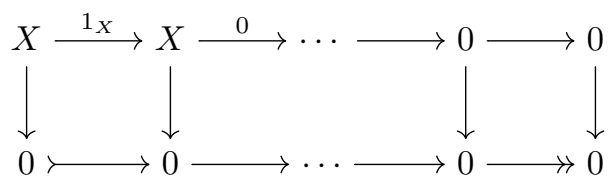

Moreover, one can easily show that two contractible $n$-exact sequences with the same end terms are weakly isomorphic, hence Proposition 4.6 implies that all contractible $n$-exact sequences are admissible. Also, it is straightforward to verify that the class of all contractible $n$-exact sequences in an additive category $\mathcal{M}$ is an $n$-exact structure; in fact, this is the smallest $n$-exact structure on $\mathcal{M}$. In particular, every additive category can be considered as an $n$-exact category with a "contractible $n$-exact structure", for each positive integer $n$. 
The following characterization of $n$-pushout diagrams of $n$-exact sequences should be compared with [18, Prop. 2.12].

Proposition 4.8. Let $(\mathcal{M}, \mathcal{X})$ be an n-exact category. Suppose that we are given a commutative diagram



in which the top row is an admissible $n$-exact sequence and $d_{Y}^{0}$ is an admissible monomorphism. Then the following statements are equivalent:

(i) Diagram 4.1] is an n-pushout diagram.

(ii) The mapping cone of diagram (4.1) is an admissible n-exact sequence.

(iii) Diagram (4.1) is both an n-pushout and an n-pullback diagram.

(iv) There exists a commutative diagram

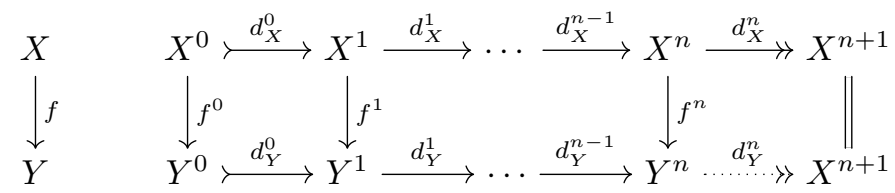

whose rows are admissible $n$-exact sequences.

Proof. (i) implies (iii). This is an adaptation of the proof of [18, Prop. 2.12]. Since the leftmost $n$ squares in (4.1) form an $n$-pushout diagram, by definition its mapping cone gives an $n$-cokernel of the morphism

$$
d_{C}^{-1}=\left[\begin{array}{ll}
-d_{X}^{0} & f^{0}
\end{array}\right]^{\top}: X^{0} \rightarrow X^{1} \oplus Y^{0} .
$$

Hence, by Lemma 4.5 it is sufficient to show that $d_{C}^{-1}$ is an admissible monomorphism. For this, observe that $d_{C}^{-1}$ equals the composition

$$
X^{0} \stackrel{\left[\begin{array}{l}
1 \\
0
\end{array}\right]}{\longrightarrow} X^{0} \oplus Y^{0} \stackrel{\left[\begin{array}{cc}
-1 & 0 \\
f^{0} & 1
\end{array}\right]}{\sim} X^{0} \oplus Y^{0} \stackrel{\left[\begin{array}{cc}
d_{X}^{0} & 0 \\
0 & 1
\end{array}\right]}{\longrightarrow} X^{1} \oplus Y^{0}
$$

where the rightmost morphism is an admissible monomorphism by Proposition 4.6 and the remaining morphisms are admissible monomorphisms by Remark 4.7. Thus $d_{C}^{-1}$ is an admissible monomorphism by axiom (E1).

That (ii) implies (iii) follows directly from the definitions of $n$-pushout and $n$ pullback diagrams. That (iii) implies (i) is obvious. Therefore statements (ii), (ii) and (iii) are equivalent.

(i) implies (iv). We begin by constructing the morphism $d_{Y}^{n}: Y^{n} \rightarrow X^{n+1}$. Since $d_{C}^{n-1}$ is a cokernel of $d_{C}^{n-2}$ (here $C$ is the mapping cone of $f$ ), there exists a unique morphism $d_{Y}^{n}: Y^{n} \rightarrow X^{n+1}$ such that $d_{X}^{n}=f^{n} d_{Y}^{n}$ and $d_{Y}^{n-1} d_{Y}^{n}=0$, see (2.4). Since $d_{X}^{n}$ is an epimorphism, it follows immediately that so is $d_{Y}^{n}$. It remains to show that $d_{Y}^{n}$ is a cokernel of $d_{Y}^{n-1}$. For this, let $u: Y^{n} \rightarrow M$ be a morphism such that $d_{Y}^{n-1} u=0$. Then we have that

$$
d_{X}^{n-1}\left(f^{n} u\right)=\left(f^{n-1} d_{Y}^{n-1}\right) u=0 .
$$


Since $d_{X}^{n}$ is a cokernel of $d_{X}^{n-1}$, there exists a morphism $v: X^{n+1} \rightarrow M$ such that $f^{n} u=d_{X}^{n} v$. It follows that

$$
f^{n} u=d_{X}^{n} v=f^{n}\left(d_{Y}^{n} v\right)
$$

and

$$
d_{Y}^{n-1} u=0=d_{Y}^{n-1}\left(d_{Y}^{n} v\right)
$$

Since $d_{C}^{n-1}$ is a cokernel of $d_{C}^{n-2}$, we have $u=d_{Y}^{n} v$. This shows that the epimorphism $d_{Y}^{n}$ is a cokernel of $d_{Y}^{n-1}$.

Let $2 \leq k \leq n$. We need to show that $d_{Y}^{k}$ is a weak cokernel of $d_{Y}^{k-1}$. Let $u: Y^{k} \rightarrow M$ be a morphism such that $d_{Y}^{k-1} u=0$. Then we have

$$
d_{X}^{k-1}\left(f^{k} u\right)=\left(f^{k-1} d_{Y}^{k-1}\right) u=0 .
$$

Since $d_{X}^{k}$ is a weak cokernel of $d_{X}^{k-1}$, there exists a morphism $v: X^{k+1} \rightarrow M$ such that $f^{k} u=d_{X}^{k} v$. Hence, given that $d_{C}^{k-1}$ is a weak cokernel of $d_{C}^{k-2}$, there exists a morphism $w: Y^{k+1} \rightarrow M$ such that $d_{Y}^{k} w=u$, see 2.4. Therefore $d_{Y}^{k}$ is a weak cokernel of $d_{Y}^{k-1}$, as required. This shows that $\left(d_{Y}^{1}, \ldots, d_{Y}^{n}\right)$ is an $n$-cokernel of $d_{Y}^{0}$, so we have finished the construction of the required commutative diagram. Moreover, by Lemma 4.5, the sequence $Y$ is an admissible $n$-exact sequence.

(iv) implies (ii). We assume that $n \geq 2$. The case $n=1$ can be shown by combining the arguments below, and is easily found in the literature, see for example [18. Prop. 2.12]. By definition, we need to show that in the mapping cone $C=C(f)$ we have that $\left(d_{C}^{0}, d_{C}^{1}, \ldots, d_{C}^{n}\right)$ is an $n$-cokernel of $d_{C}^{-1}$.

Let $2 \leq k \leq n$. We shall show that $d_{C}^{k-1}$ is a weak cokernel of $d_{C}^{k-2}$, see (2.4). Indeed, let $u: Y^{k-1} \rightarrow M$ and $v: X^{k} \rightarrow M$ be morphisms such that $d_{Y}^{k-2} u=0$ and $d_{X}^{k-1} v=f^{k-1} u$. Since $d_{Y}^{k-1}$ is a weak cokernel of $d_{Y}^{k-2}$, there exists a morphism $w: Y^{k} \rightarrow M$ such that $u=d_{Y}^{k-1} w$. Moreover, note that

$$
d_{X}^{k-1} v=f^{k-1} u=f^{k-1}\left(d_{Y}^{k-1} w\right)=d_{X}^{k-1}\left(f^{k} w\right)
$$

for $f$ is a morphism of complexes. Given that $d_{X}^{k}: X^{k} \rightarrow X^{k+1}$ is a weak cokernel of $d_{X}^{k-1}: X^{k-1} \rightarrow X^{k}$, there exists a morphism $h^{k+1}: X^{k+1} \rightarrow M$ such that $f^{k} w-v=$ $d_{X}^{k} h^{k+1}$. If $k \neq n$, then the claim follows. If $k=n$, then let $w^{\prime}:=w-d_{Y}^{n} h^{n+1}$. It follows that $d_{Y}^{n-1} w^{\prime}=d_{Y}^{n-1} w=u$ and

$$
f^{n} w^{\prime}=f^{n} w-f^{n} d_{Y}^{n} h^{n+1}=v+d_{X}^{n} h^{k+1}-d^{n} h^{k+1}=v .
$$

This shows that $d_{C}^{n-1}$ is a weak cokernel of $d_{C}^{n-2}$.

We need to show that $d_{C}^{n-1}$ is a cokernel of $d_{C}^{n-2}$. For this it is enough to show that $d_{C}^{n-1}$ is an epimorphism. Let $p: Y^{n} \rightarrow M$ be a morphism such that $d_{Y}^{n-1} p=0$ and $f^{n} p=0$. Then, since $d_{Y}^{n}$ is a cokernel of $d_{Y}^{n-1}$, there exists a morphism $q: X^{n+1} \rightarrow M$ such that $p=d_{Y}^{n} q$. Thus,

$$
d_{X}^{n} q=\left(f^{n} d_{Y}^{n}\right) q=f^{n} p=0
$$

Since $d_{X}^{n}$ is an epimorphism, we have $q=0$ which implies that $p=0$. This shows that $d_{C}^{n-1}$ is an epimorphism.

It remains to show that $d_{C}^{0}$ is a weak cokernel of $d_{C}^{-1}$. Let $y: Y^{0} \rightarrow Z^{0}$ and $v: X^{0} \rightarrow Z^{0}$ be morphisms such that $f^{0} u=d_{X}^{0} v$. By axiom (E2) there exists an 
$n$-pushout diagram of $\left(d_{Y}^{0}, \ldots, d_{Y}^{n-1}\right)$ along $u$. Moreover, since we have shown the implication from (i) to (iv), we can construct a commutative diagram

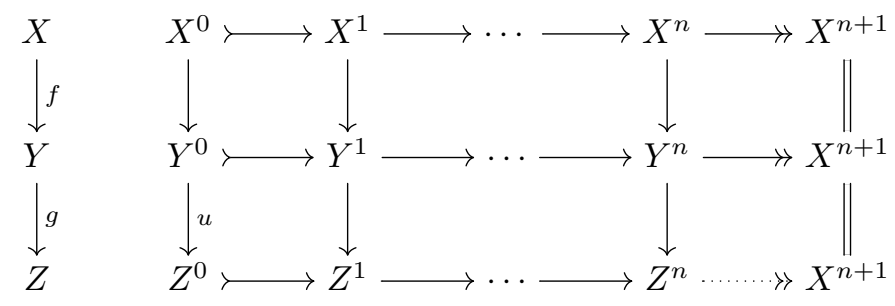

where the leftmost $n$ squares of the two bottom rows form a pushout diagram. It follows that the following diagram commutes

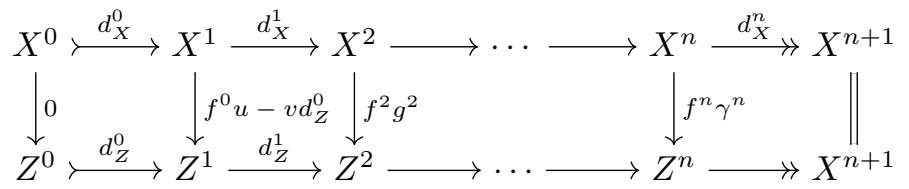

Then, by the Comparison Lemma 2.1, there exists a morphism $h: X^{n+1} \rightarrow Z^{n}$ such that $h d_{Z}^{n}=1_{X^{n+1}}$. Therefore $d_{Z}^{n+1}$ is a split epimorphism. From the dual of Proposition 2.6 we conclude that $d_{Z}^{0}$ is a split monomorphism. It follows that there exists a morphism $w: Z^{1} \rightarrow Z^{0}$ such that $d_{Z}^{0} w=1_{Z^{0}}$. Finally,

$$
d_{Y}^{0}\left(g^{1} w\right)=u\left(d_{Z}^{0} w\right)=u
$$

and

$$
f^{0}(u w)-d_{X}^{0}(v w)=\left(f^{0} u-d_{X}^{0} v\right) w=\left(v d_{Z}^{0}\right) w=v
$$

which is what we needed to show. This concludes the proof.

The following property is a refinement of Proposition 2.13 .

Proposition 4.9. Let $(\mathcal{M}, X)$ be an n-exact category and $g: X \rightarrow Z$ a morphism of admissible n-exact sequences. Then, for every morphism of admissible $n$-exact sequences $g: X \rightarrow Y$ there exists a commutative diagram

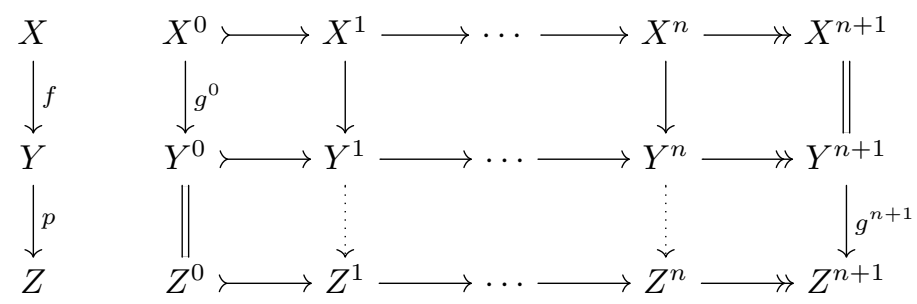

where $f^{0}=g^{0}$ and $p^{n+1}=g^{n+1}$. Moreover, there exists a homotopy $h: f p \rightarrow g$ with $h^{1}=0$ and $h^{n+1}=0$.

Proof. By Proposition 4.8 the morphism of admissible $n$-exact sequences $f$ exists. Then, by Proposition 2.13, we only need to show that $p^{n} d_{Z}^{n}=d_{Y}^{n} g^{n+1}$. Indeed, on one hand we have

$$
f^{n}\left(g^{n} d_{Z}^{n}\right)=d_{X}^{n} g^{n+1}=f^{n}\left(d_{Y}^{n} g^{n+1}\right) .
$$


On the other hand,

$$
d_{Y}^{n-1}\left(g^{n} d_{Z}^{n}\right)=g^{n-1}\left(d_{Z}^{n-1} d_{Z}^{n}\right)=0=d_{Y}^{n-1}\left(d_{Y}^{n} g^{n+1}\right) .
$$

Since in the mapping cone $C(f)$ we have that $d_{C}^{n-1}$ is a cokernel of $d_{C}^{n-2}$, we have $p^{n} d_{Z}^{n}=d_{Y}^{n} g^{n+1}$, see 2.4. This concludes the proof.

The next result shows that, in an $n$-exact category, equivalences of admissible $n$ exact sequences induce isomorphisms in the homotopy category, cf. Proposition 2.7 and the remark after it.

Proposition 4.10. Let $(\mathcal{M}, X)$ be an n-exact category, $f: X \rightarrow Y$ an equivalence of admissible $n$-exact sequences. Then, there exists an equivalence of $n$-exact sequences $g: Y \rightarrow X$ such that $f$ and $g$ are mutually inverse isomorphisms in $\mathrm{H}(\mathcal{M})$.

Proof. By Proposition 4.8, the mapping cone $C=C(f)$ of the diagram

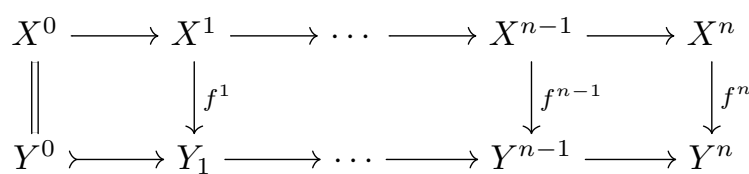

is an admissible $n$-exact sequence. Since $d_{C}^{-1}: X^{0} \rightarrow X^{1} \oplus X^{0}$ is a split monomorphism, $C$ is a contractible $n$-exact sequence, see Proposition 2.6. Hence there exists a null-homotopy $h: 1_{C} \rightarrow 0$. It is straightforward to verify that $h$ induces an equivalence of admissible $n$-exact sequences $g: Y \rightarrow X$. Finally, the Comparison Lemma 2.1 implies that $f$ and $g$ induce mutually inverse isomorphisms in $\mathrm{H}(\mathcal{M})$.

By axiom (E1) the class of admissible monomorphisms in an $n$-exact category is closed under composition. The next result, an analog of Quillen's obscure axiom for exact categories, shows that a partial converse also holds, cf. [18, Prop. 2.16].

Proposition 4.11 (Obscure axiom). Let $(\mathcal{M}, \mathcal{X})$ be an n-exact category. Suppose there is a commutative diagram

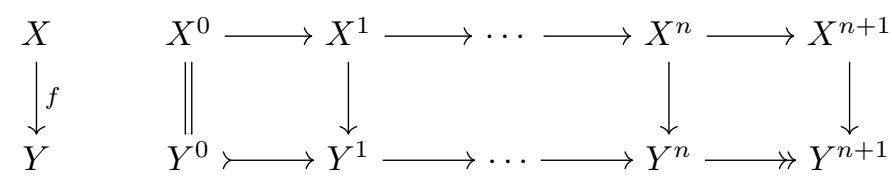

where the bottom row is an admissible $n$-exact sequence and $\left(d_{X}^{1}, \ldots, d_{X}^{n}\right)$ is an $n$-cokernel of $d_{X}^{0}$. Then, the top row is also an admissible $n$-exact sequence.

Proof. This is an adaptation of the proof of [18, Prop. 2.16], which is due to Keller. Since a pushout of the bottom row along $d_{X}^{0}$ exists, by Proposition 4.8 the morphism $\left[\begin{array}{ll}-d_{Y}^{0} & d_{X}^{0}\end{array}\right]^{\top}: X^{0} \rightarrow Y^{1} \oplus X^{1}$ is an admissible monomorphism. Moreover, the diagram

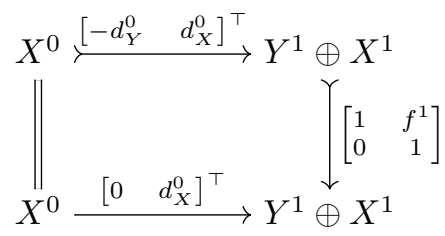


commutes. Hence $\left[0 d_{X}^{0}\right]^{\top}: X^{0} \rightarrow Y^{1} \oplus X^{1}$ is the composition of an admissible monomorphism with an isomorphism and, by axiom (E1) and Remark 4.7, we conclude that itself is an admissible monomorphism. Using the factorization property of weak cokernels we can construct a commutative diagram

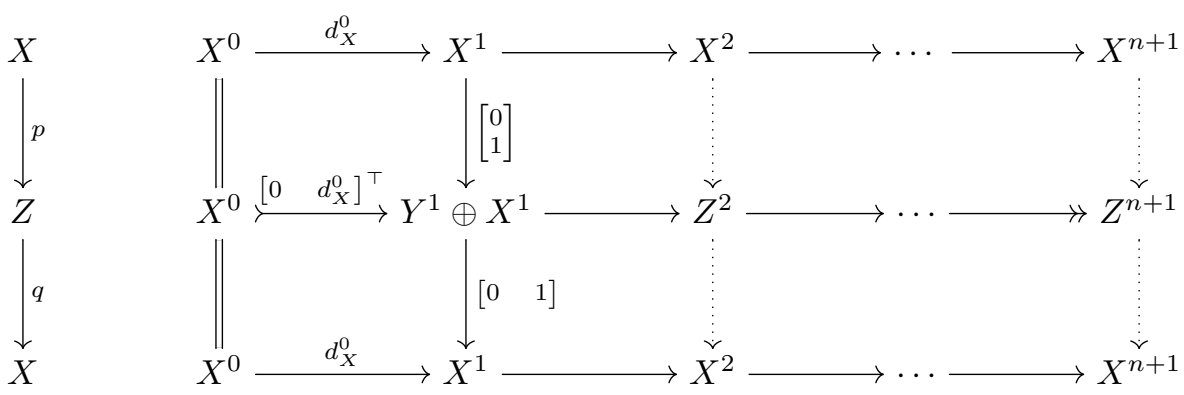

where the middle row is an admissible $n$-exact sequence. By the Comparison Lemma 2.1 applied to the compositions $p q$ and $q p$, the morphisms $p$ and $q$ induce mutually inverse isomorphisms in $\mathrm{H}(\mathcal{M})$. By Proposition 2.5 we have that $X$ is an $n$-exact sequence. Then, Proposition 4.9 gives an equivalence between $X$ and an admissible $n$-exact sequence which is obtained by $n$-pullback from $Y$ along $f^{n+1}$. Since $X$ is closed under weak isomorphisms (in particular, equivalences) of $n$-exact sequences, we have that $X$ is moreover an admissible $n$-exact sequence.

The following result shows that the class of $n$-exact sequences in an $n$-exact category is closed under direct summands.

Proposition 4.12. Let $(\mathcal{M}, X)$ be an n-exact category, and $X$ and $Y$ complexes in $\mathrm{e}^{n}(\mathrm{C})$. If $X \oplus Y$ is an $n$-exact sequence, then so are $X$ and $Y$.

Proof. This is an adaptation of the proof of [18, Cor. 2.18]. Clearly, $X$ and $Y$ are $n$-exact sequences. In particular, $d_{X}^{0}$ admits an $n$-cokernel. Moreover, we have a commutative diagram

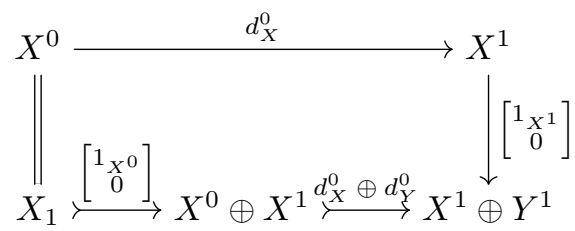

in which the composition along the bottom row yields an admissible monomorphism by (E1)] We conclude from Proposition 4.11 that $X$ is an admissible $n$-exact sequence. Analogously one can show that $Y$ is an admissible $n$-exact sequence.

4.2. $n$-exact categories and cluster-tilting. Let $(\mathcal{E}, X)$ be an exact category. Recall that a morphism $X \rightarrow Y$ in $\mathcal{E}$ is proper if it has a factorization $X \rightarrow Z \mapsto Y$ through an $X$-admissible epimorphism and an $X$-admissible monomorphism. A sequence of proper morphisms

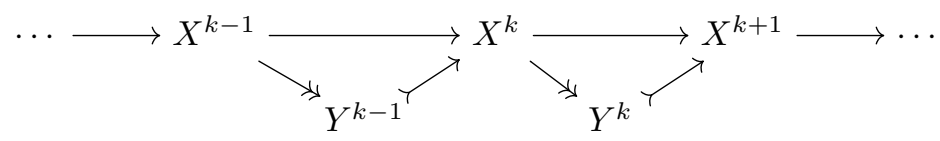





$X$-admissible exact sequence. If the class $X$ is clear from the complex, then we may write "acyclic" instead of " $X$-acyclic". We denote the full subcategory of $H(\mathcal{E})$ given by the acyclic complexes by Ac.

Following Neeman [41, the derived category $\mathcal{D}=\mathrm{D}(\mathcal{E}, X)$ is by definition the Verdier quotient $\mathrm{H}(\mathcal{E}) / \operatorname{thick}(\operatorname{Ac}(X))$. Then, for all $k \geq 1$ and for all $E \in \mathcal{E}$ we can define the functor $\operatorname{Ext}_{X}^{k}(E,-): \mathcal{E} \rightarrow \operatorname{Mod} \mathbb{Z}$ by $F \mapsto \operatorname{Hom}_{\mathcal{D}}(E, F[k])$. Equivalently, one can define $\operatorname{Ext}_{x}^{k}(E, F)$ as the set of Yoneda equivalence classes of Yoneda splicings of $X$-admissible exact sequences in the usual way, see [20, Sec. 6].

We use the following variant of the definition of $n$-cluster-tilting subcategory of an exact category. Note that in the case of abelian categories this definition agrees with Definition 3.14, see Remark 3.15.

Definition 4.13. Let $(\mathcal{E}, \mathcal{X})$ be a small exact category and $\mathcal{N}$ a subcategory of $\mathcal{E}$. We say that $\mathcal{M}$ is an $n$-cluster-tilting subcategory of $(\mathcal{E}, \mathcal{X})$ if the following conditions are satisfied:

(i) Every object $E \in \mathcal{E}$ has a left $\mathcal{M}$-approximation by an $\mathcal{X}$-admissible monomorphism $E \longmapsto M$.

(ii) Every object $E \in \mathcal{E}$ has a right $\mathcal{M}$-approximation by an $\mathcal{X}$-admissible epimorphism $M^{\prime} \rightarrow E$.

(iii) We have

$$
\begin{aligned}
\mathcal{M} & =\left\{E \in \mathcal{E} \mid \text { for all } i \in\{1, \ldots, n-1\} \operatorname{Ext}_{x}^{i}(E, \mathcal{M})=0\right\} \\
& =\left\{E \in \mathcal{E} \mid \text { for all } i \in\{1, \ldots, n-1\} \operatorname{Ext}_{x}^{i}(\mathcal{M}, E)=0\right\} .
\end{aligned}
$$

Note that $\mathcal{E}$ itself is the unique 1-cluster-tilting subcategory of $\mathcal{E}$.

Our aim in this section is to prove the following result, which is analogous to Theorem 3.16 in the case of exact categories.

Theorem 4.14. Let $(\mathcal{E}, \mathcal{X})$ be an exact category and $\mathcal{M}$ an $n$-cluster-tilting subcategory of $(\mathcal{E}, X)$. Let $\mathcal{Y}=\mathcal{Y}(\mathcal{M}, X)$ be the class of all $X$-acyclic complexes

$$
X^{0} \longmapsto X^{1} \longrightarrow \cdots \longrightarrow X^{n} \longrightarrow X^{n+1}
$$

such that for all $k \in\{0,1, \ldots, n+1\}$ we have $X^{k} \in \mathcal{M}$. Then $(\mathcal{M}, y)$ is an n-exact category.

To prove Theorem 4.14, we need the following result, whose proof is completely analogous to the proof of Proposition 3.17 .

Proposition 4.15. Let $(\mathcal{E}, \mathcal{X})$ be an exact category and $\mathcal{M}$ an $n$-cluster-tilting subcategory of $\mathcal{E}$. Then, for each $E \in \mathcal{E}$ there exists an acyclic complex of the form

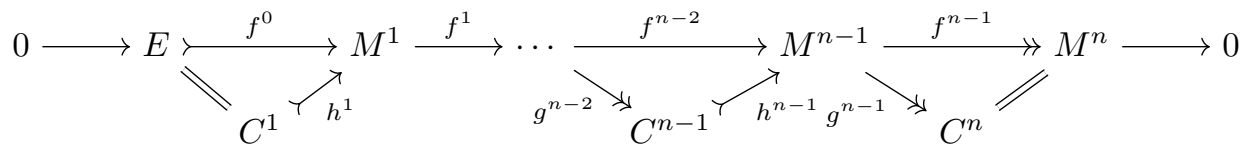

satisfying the following properties:

(i) For each $k \in\{1, \ldots, n\}$ we have $M^{k} \in \mathcal{M}$.

(ii) For each $k \in\{1, \ldots, n-1\}$ the morphism $h^{k}: C^{k} \longmapsto M^{k}$ is an $X$ admissible monomorphism and a left $\mathcal{M}$-approximation of $C^{k}$. 
(iii) For each $k \in\{1, \ldots, n-1\}$ the pair $C^{k} \longmapsto M^{k} \rightarrow C^{k+1}$ is an $X_{\text {- }}$ admissible exact sequence.

We now give the proof of Theorem 4.14 .

Proof of Theorem 4.14. If $n=1$, then the result is trivial. Let $n \geq 2$. We observe that the class $y$ indeed consists of $n$-exact sequences. To see this, given $X \in \mathcal{y}$ and $M \in \mathcal{M}$, apply the functor $\mathcal{E}(M,-)$ to $X$ to obtain a sequence

$$
0 \longrightarrow \mathcal{E}\left(M, X^{0}\right) \longrightarrow \mathcal{E}\left(M, X^{1}\right) \longrightarrow \cdots \longrightarrow \mathcal{E}\left(M, X^{n}\right) \longrightarrow \mathcal{E}\left(M, X^{n+1}\right)
$$

which is exact at $\mathcal{E}\left(M, X^{0}\right)$ and $\mathcal{E}\left(M, X^{1}\right)$. By the version of the dual of Proposition 3.18 for exact categories, for each $k \in\{1, \ldots, n-1\}$ the homology of this complex at $\mathcal{E}\left(M, X^{k+1}\right)$ is isomorphic to $\operatorname{Ext}_{x}^{k}\left(M, X^{0}\right)$ which vanishes by assumption. Combining with the dual argument, this shows that $X$ is an $n$-exact sequence.

We only show that $(\mathcal{M}, y)$ satisfies axioms (E1) and (E2) in Definition 4.2 since the remaining axioms are dual. Note that axiom (E0) is obviously satisfied.

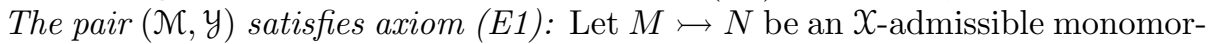
phism such that $M, N \in \mathcal{M}$ and $N \rightarrow E$ a cokernel of $M \longmapsto N$. Applying Proposition 4.15 to $N$ yields an acyclic complex in $y$ with $M \longmapsto N$ as the leftmost morphism. This shows that the class of $y$-admissible monomorphisms coincides with the class of $\mathcal{X}$-admissible monomorphisms. Therefore $(\mathcal{M}, \mathcal{Y})$ satisfies axiom (E1) That $(\mathcal{M}, y)$ satisfies axiom $\left(\mathrm{E} 1^{\mathrm{op}}\right)$ then follows by duality.

The pair $(\mathcal{M}, y)$ satisfies axiom (E2): Let $X$ be a $y$-admissible $n$-exact sequence and $f^{0}: X^{0} \rightarrow Y^{0}$ a morphism in $\mathcal{\mathcal { M }}$. We need to construct a commutative diagram

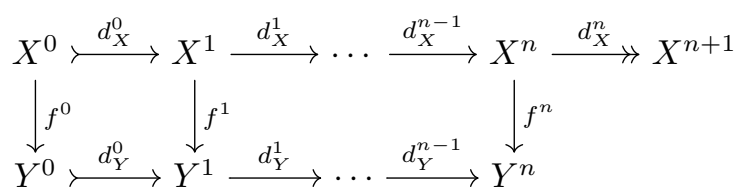

such that $d_{Y}^{0}$ is a $y$-admissible monomorphism or, equivalently by the previous paragraph, an $X$-admissible monomorphism.

Step 1: We claim that there is a $y$-admissible $n$-exact sequence

$$
C: \quad X^{0} \longleftrightarrow X^{1} \oplus Y^{0} \longrightarrow X^{n} \oplus Y^{n-1} \longrightarrow Y^{n}
$$

with differentials

$$
d_{C}^{k-1}:=\left[\begin{array}{cc}
-d_{X}^{k} & 0 \\
f^{k} & d_{Y}^{k-1}
\end{array}\right]: X^{k} \oplus Y^{k-1} \longrightarrow X^{k+1} \oplus Y^{k} .
$$

We shall construct $C$ inductively. Set $Z^{0}:=X^{0}, u^{0}:=1_{X^{0}}, p^{0}:=-d_{X}^{0}$ and $q^{0}:=f^{0}$. Let $0 \leq k \leq n-2$ and suppose that we have constructed a commutative diagram

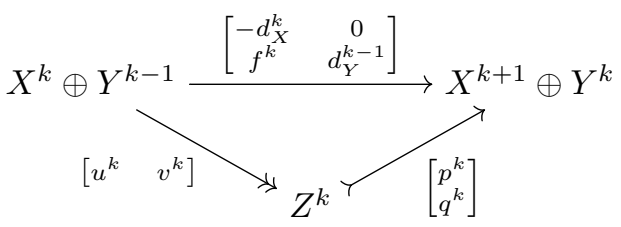


Since $Z^{k} \longmapsto X^{k+1} \oplus Y^{k}$ is an $X$-admissible monomorphism, there exist morphisms $u^{k+1}: X^{k+1} \rightarrow Z^{k+1}$ and $v^{k+1}: Y^{k} \rightarrow Z^{k+1}$ such that $\left[u^{k+1} v^{k+1}\right]: X^{k+1} \oplus Y^{k} \rightarrow$ $Z^{k+1}$. Therefore the square

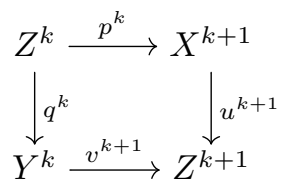

is a pushout diagram.

It is readily verified that the composition

$$
\left.X^{k} \oplus Y^{k-1} \stackrel{\left[\begin{array}{cc}
-d_{X}^{k} & 0 \\
f^{k} & d_{Y}^{k-1}
\end{array}\right]}{\longrightarrow} X^{k+1} \oplus Y^{k} \stackrel{\left[d_{X}^{k+1}\right.}{0}\right]\left[X^{k+2}\right.
$$

is zero. Then, given that $X^{k} \oplus Y^{k-1} \rightarrow Z^{k}$ is an epimorphism, the composition

$$
\left.Z^{k} \stackrel{\left[\begin{array}{c}
p^{k} \\
q^{k}
\end{array}\right]}{\longrightarrow} X^{k+1} \oplus Y^{k} \stackrel{\left[d_{X}^{k+1}\right.}{0}\right] \longrightarrow X^{k+2}
$$

is also zero. Hence we have $p^{k} d_{X}^{0}=0$.

Since 4.6 is a pushout diagram, there exists a morphism $p^{k+1}: Z^{k+1} \rightarrow X^{k+2}$ such that $u^{k+1} p^{k+1}=-d_{X}^{k+1}$ and $v^{k+1} p^{k+1}=0$. Let $q^{k+1}: Z^{k+1} \longmapsto Y^{k+1}$ be a left

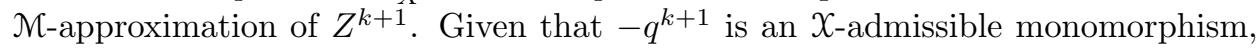
Proposition 4.8 applied to the exact category $(\mathcal{E}, X)$ implies that the morphism $\left[q^{k+1} p^{k+1}\right]: Z^{k+1} \longmapsto X^{k+2} \oplus Y^{k+1}$ is an $X$-admissible monomorphism. Set $f^{k+1}:=$ $u^{k+1} q^{k+1}$ and $d_{Y}^{k}:=v^{k+1} q^{k+1}$. It is readily verified that the following diagram commutes:



Finally, let $d_{C}^{n-1}: X^{n} \oplus Y^{n-1} \rightarrow Y^{n}$ be a cokernel of $Z^{n-1} \longmapsto X^{n} \oplus Y^{n-1}$. It follows that $C \in \mathcal{y}$, and hence is an $n$-exact sequence.

Step 2: We claim that the morphism $d_{Y}^{0}$ is a $y$-admissible monomorphism. Indeed, we have $d_{Y}^{0}=v^{1} q^{1}$. Moreover, $v^{1}$ is an $X$-admissible monomorphism for it is defined by the following pushout diagram in the exact category $(\mathcal{E}, X)$ :



Therefore $d_{Y}^{0}$ is the composition of two $X$-admissible monomorphisms, hence itself is an $X$-admissible monomorphism. Since $X$-admissible monomorphisms and $y$ admissible morphisms coincide, the claim follows. The existence of a commutative

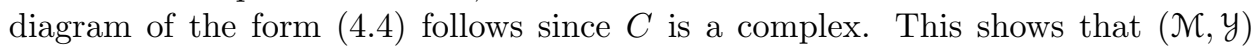
satisfies axiom (E2). That $(\mathcal{M}, y)$ satisfies axiom $\left(\mathrm{E} 2^{\mathrm{op}}\right)$ then follows by duality. 


\section{Frobenius $n$-EXACT CATEGORIES}

In this section we introduce Frobenius $n$-exact categories and show that their stable categories have the structure of an $(n+2)$-angulated category; this allows us to introduce algebraic $(n+2)$-angulated categories. We give a method to construct Frobenius $n$-exact categories (and thus also algebraic $n$-angulated categories) from certain $n$-cluster-tilting subcategories of Frobenius exact categories. We show that our construction is closely related to the standard construction of $(n+2)$-angulated categories given in [21, Thm. 1], see Theorem 5.16.

5.1. Reminder on $(n+2)$-angulated categories. We follow the exposition of [21, Sec. 2] although we prefer to set the base case of triangulated categories as the case $n=1$, in agreement with our convention for abelian and exact categories. Thus we consider $(n+2)$-angulated categories instead of ' $n$-angulated categories' as done in loc.cit..

Let $n$ be a positive integer and $\mathcal{F}$ an additive category equipped with an automorphism $\Sigma: \mathcal{F} \rightarrow \mathcal{F}$. An $n$ - $\Sigma$-sequence in $\mathcal{F}$ is a sequence of morphisms

$$
X^{0} \stackrel{\alpha^{0}}{\longrightarrow} X^{1} \stackrel{\alpha^{1}}{\longrightarrow} X^{2} \stackrel{\alpha^{2}}{\longrightarrow} \cdots \stackrel{\alpha^{n}}{\longrightarrow} X^{n+1} \stackrel{\alpha^{n+1}}{\longrightarrow} \Sigma X^{0} .
$$

Its left rotation is the $n$ - $\Sigma$-sequence

$$
X^{1} \stackrel{\alpha^{1}}{\longrightarrow} X^{2} \stackrel{\alpha^{2}}{\longrightarrow} \cdots \stackrel{\alpha^{n}}{\longrightarrow} X^{n+1} \stackrel{\alpha^{n+1}}{\longrightarrow} \Sigma X^{0} \stackrel{(-1)^{n} \Sigma \alpha^{0}}{\longrightarrow} \Sigma X^{1}
$$

A morphism of $n$ - $\Sigma$-sequences is a commutative diagram

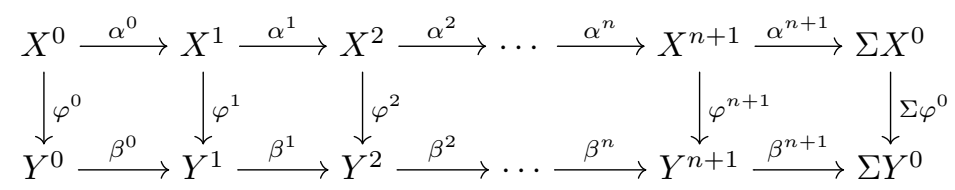

where each row is an $n$ - $\Sigma$-sequence. The mapping cone $C(\varphi)$ of the above morphism is the $n$ - $\Sigma$-sequence

$$
X^{1} \oplus Y^{0} \stackrel{\gamma^{0}}{\longrightarrow} X^{2} \oplus Y^{1} \stackrel{\gamma^{1}}{\longrightarrow} \cdots \stackrel{\gamma^{n}}{\longrightarrow} \Sigma X^{0} \oplus Y^{n+1} \stackrel{\gamma^{n+1}}{\longrightarrow} \Sigma X^{1} \oplus \Sigma Y^{0}
$$

where for each $k \in\{0, \ldots, n\}$ we define

$$
\gamma^{k}:=\left[\begin{array}{cc}
-\alpha^{k+1} & 0 \\
\varphi^{k+1} & \beta^{k}
\end{array}\right]: X^{k+1} \oplus Y^{k} \longrightarrow X^{k+2} \oplus Y^{k+1}
$$

(by convention, $\alpha^{n+2}:=\Sigma \alpha^{0}, \varphi^{n+2}:=\Sigma \varphi^{0}$ ). A weak isomorphism is a morphism of $n$ - $\Sigma$-sequences such that $\varphi^{k}$ and $\varphi^{k+1}$ are isomorphisms for some $k \in$ $\{0,1, \ldots, n+1\}$. Abusing the terminology, we say that two $n$ - $\Sigma$-sequences are weakly isomorphic if they are connected by a finite zigzag of weak isomorphisms.

Definition 5.1. 21] A pre- $(n+2)$-angulated category is a triple $(\mathcal{F}, \Sigma, \mathcal{S})$ where $\mathcal{F}$ is an additive category, $\Sigma: \mathcal{F} \rightarrow \mathcal{F}$ is an automorphism 1 and $\mathcal{S}$ is a class of $n$ $\Sigma$-sequences (whose members we call $(n+2)$-angles) which satisfies the following axioms:

\footnotetext{
1 One may consider the more general case when $\Sigma: \mathcal{F} \rightarrow \mathcal{F}$ is an autoequivalence. As mentioned in [21 $2.2 \mathrm{Rmks}$.], it can be shown that the assumption of $\Sigma$ being invertible is but a mild sacrifice, cf. [39, Sec. 2].
} 
(F1) The class $\mathcal{S}$ is closed under taking direct summands and making direct sums. For all $X \in \mathcal{F}$ the trivial sequence

$$
X \stackrel{1_{X}}{\longrightarrow} X \longrightarrow 0 \longrightarrow \cdots \longrightarrow x
$$

belongs to $\mathcal{S}$. For each morphism $\alpha$ in $\mathcal{M}$ there exists an $n$-angle whose first morphism is $\alpha$.

(F2) An $n$ - $\Sigma$-sequence of the form (5.1) is a $(n+2)$-angle if and only if its left rotation is a $(n+2)$-angle.

(F3) Each commutative diagram

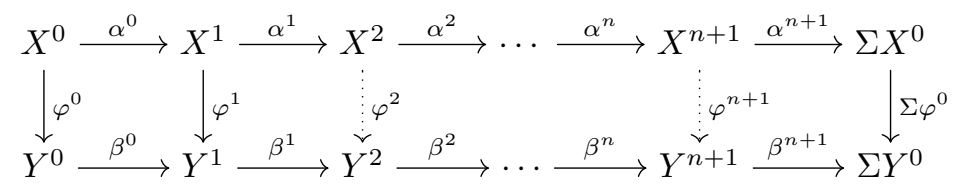

whose rows are $(n+2)$-angles can be completed to a morphism of $n$ - $\Sigma$ sequences.

We call the class $\mathcal{S}$ a pre- $(n+2)$-angulation of $\mathcal{F}$ with respect to $\Sigma$. If moreover the following axiom is satisfied, then $(\mathcal{F}, \Sigma, \mathcal{S})$ is called an $n$-angulated category and the class $\mathcal{S}$ is called a $(n+2)$-angulation.

(F4) In the situation of axiom (F3), the morphisms $\varphi^{2}, \ldots, \varphi^{n+1}$ can be chosen in such a way that the mapping cone $C(\varphi)$ is a $(n+2)$-angle.

An $n$ - $\Sigma$-sequence $X$ is exact if for all $F \in \mathcal{F}$ and for all $k \in \mathbb{Z}$ the induced sequence of abelian groups

$$
\mathcal{F}\left(F, \Sigma^{k-1} X^{n+1}\right) \longrightarrow \mathcal{F}\left(F, \Sigma^{k} X^{0}\right) \longrightarrow \cdots \longrightarrow \mathcal{F}\left(F, \Sigma^{k} X^{n+1}\right) \longrightarrow \mathcal{F}\left(F, \Sigma^{k+1} X^{0}\right)
$$

is exact. We need the following result.

Proposition 5.2. 21, Prop. 2.5(c)] Let $(\mathcal{F}, \Sigma, \mathcal{S})$ be a pre- $(n+2)$-angulated category. If $\mathcal{S}^{\prime}$ is a pre- $(n+2)$-angulation of $\mathcal{F}$ such that $\mathcal{S}^{\prime} \subseteq \mathcal{S}$, then $\mathcal{S}=\mathcal{S}^{\prime}$.

Let $\left(\mathcal{F}, \Sigma_{\mathcal{F}}, \mathcal{S}_{\mathcal{F}}\right)$ and $\left(\mathcal{G}, \Sigma_{\mathcal{G}}, \mathcal{S}_{\mathcal{G}}\right)$ be $(n+2)$-angulated categories. An exact functor is a pair $(F, \eta)$ wehre $F: \mathcal{F} \rightarrow \mathcal{G}$ is an additive functor and $\eta: F \Sigma_{\mathcal{F}} \rightarrow \Sigma_{\mathcal{G}} F$ is a natural transformation such that for every $(n+2)$-angle in $\mathcal{F}$

$$
X^{0} \stackrel{\alpha^{0}}{\longrightarrow} X^{1} \stackrel{\alpha^{1}}{\longrightarrow} X^{2} \stackrel{\alpha^{2}}{\longrightarrow} \cdots \stackrel{\alpha^{n}}{\longrightarrow} X^{n+1} \stackrel{\alpha^{n+1}}{\longrightarrow} \Sigma_{\mathcal{F}} X^{0}
$$

we have that

$$
F X^{0} \stackrel{F \alpha^{0}}{\longrightarrow} F X^{1} \stackrel{F \alpha^{1}}{\longrightarrow} F X^{2} \stackrel{F \alpha^{2}}{\longrightarrow} \cdots \stackrel{F \alpha^{n}}{\longrightarrow} F X^{n+1} \stackrel{\beta}{\longrightarrow} \Sigma_{\mathcal{G}} F X^{0}
$$

is a $(n+2)$-angle in $\mathcal{G}$, where $\beta:=\left(F \alpha^{n+1}\right) \eta_{X^{0}}$.

5.2. Frobenius $n$-exact categories and algebraic $(n+2)$-angulated categories. Our approach in this subsection is analogous to [25, Sec. I.2].

Definition 5.3. Let $(\mathcal{N}, X)$ be an $n$-exact category. An object $I \in \mathcal{M}$ is $\mathcal{X}$-injective if for every admissible monomorphism $f: M \longmapsto N$ the sequence of abelian groups

$$
\mathcal{M}(N, I) \stackrel{f \cdot ?}{\longrightarrow} \mathcal{M}(M, I) \longrightarrow 0
$$


is exact. We say that $(\mathcal{M}, X)$ has enough $\mathcal{X}$-injectives if for every object $M \in \mathcal{M}$ there exists $X$-injective objects $I^{1}, \ldots, I^{n}$ and an admissible $n$-exact sequence

$$
M \longmapsto I^{1} \longrightarrow \cdots \longrightarrow I^{n} \longrightarrow N
$$

The notion of having enough $\mathcal{X}$-projectives is defined dually.

Remark 5.4. If $n=1$, then the notions of $X$-injectively cogenerated exact category and of an exact category with enough $X$-injectives coincide. On the other hand, if $n \geq 2$, then there are $n$-exact categories which are $X$-injectively cogenerated but do not have enough $X$-injectives. Indeed, let $\Lambda$ be a finite-dimensional selfinjective algebra. By Theorem 3.16 we know that an $n$-cluster-tilting subcategories $\mathcal{M}$ of $\bmod \Lambda$ is an injectively cogenerated $n$-abelian category. However, $\mathcal{M}$ has enough injectives if and only if $\mathcal{M}$ is stable under taking $n$-th cosyzygies, $c f$. Theorem 5.16 .

Let $(\mathcal{N}, X)$ be an $n$-exact category. For objects $M, N \in \mathcal{M}$, we denote by $I(M, N)$ the subgroup of $\mathcal{M}(M, N)$ of morphisms which factor through an $\mathcal{X}$-injective object. The $\mathcal{X}$-injectively stable category of $\mathcal{M}$, denoted by $\overline{\mathcal{M}}$, is the category with the same objects as $\mathcal{M}$ and with morphisms groups defined by

$$
\overline{\mathcal{M}}(M, N):=\mathcal{M}(M, N) / I(M, N) .
$$

If $\alpha: M \rightarrow N$ is a morphism in $\mathcal{M}$, we denote its equivalence class in $\overline{\mathcal{M}}(M, N)$ by $\bar{\alpha}$. It easy to see that $\overline{\mathcal{M}}$ is also an additive category. The $\mathcal{X}$-projectively stable category of $\mathcal{M}$, denoted by $\underline{\mathcal{M}}$, is defined dually.

Definition 5.5. We say that an $n$-exact category $(\mathcal{M}, X)$ is Frobenius if it has enough $X$-injectives, enough $X$-projectives, and if $X$-injective and $X$-projective objects coincide. In this case one has $\overline{\mathcal{M}}=\underline{\mathcal{M}}$, and we refer to this category as the stable category of $\mathcal{M}$.

Remark 5.6. In keeping the convention of the classical theory, if $(\mathcal{M}, X)$ is a Frobenius $n$-exact category, then we denote its stable category by $\underline{\mathcal{N}}$.

Our aim is to show that the stable category of a Frobenius $n$-exact category, has a natural structure of a $(n+2)$-angulated category. We begin with the construction of an autoequivalence $\Sigma: \underline{\mathcal{M}} \rightarrow \underline{\mathcal{M}}$. The following result should be compared with [25. Lemma I.2.2].

Lemma 5.7. Let $(\mathcal{M}, X)$ be an n-exact category. Suppose that we are given two admissible $n$-exact sequences $X$ and $Y$ such that $X^{0}=Y^{0}$ and, for $k \in\{1, n\}$, the objects $X^{k}$ and $Y^{k}$ are $X$-injective. Then, $X^{n+1}$ and $Y^{n+1}$ are isomorphic in $\overline{\mathcal{M}}$.

Proof. Since $X^{1}$ and $Y^{1}$ are $X$-injective and $X$ and $Y$ are admissible $n$-exact sequences, we can construct a commutative diagram

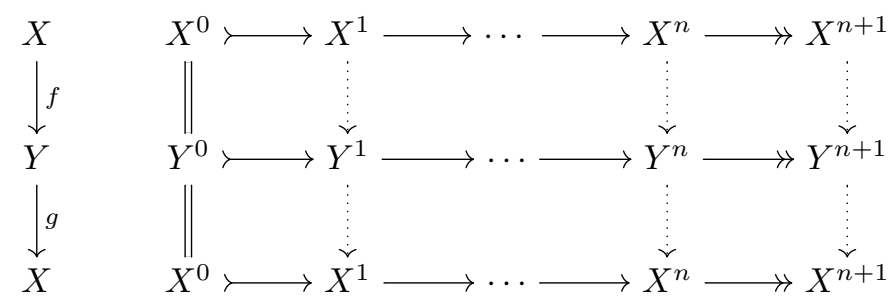


By the Comparison Lemma 2.1 there exists a morphism $h: X^{n+1} \rightarrow X^{n}$ such that

$$
f^{n+1} g^{n+1}-1=h d_{X}^{n} .
$$

Since $X^{n}$ is $X$-injective, we have $\overline{f^{n+1} g^{n+1}}=\overline{1}$. A similar argument shows that $\overline{g^{n+1} f^{n+1}}=\overline{1}$. Therefore $X^{n+1}$ and $Y^{n+1}$ are isomorphic in $\overline{\mathcal{M}}$.

Let $(\mathcal{M}, X)$ be an $n$-exact category with enough $\mathcal{X}$-injectives. For each $M \in \mathcal{M}$ we choose an admissible $n$-exact sequence

$$
I(M): M \longmapsto I^{1}(M) \longrightarrow \cdots \longrightarrow I^{n}(M) \longrightarrow S M .
$$

such that for each $k \in\{1, \ldots, n\}$ the objects $I^{k}(M)$ are $X$-injective. It follows from Lemma 5.7 that the isomorphism class of $S M$ in $\mathcal{M}$ does not depend on the choice of $I(M)$.

Let $f: M \rightarrow N$ be a morphism in $\mathcal{M}$. Since $I^{1}(N)$ is $\mathcal{X}$-injective, there is a commutative diagram of admissible $n$-exact sequences



The Comparison Lemma 2.1 implies that $\overline{S f}$ does not depend on the choices of $I^{1}(f), \ldots, I^{n}(f)$. It is readily verified that the correspondences $M \mapsto S M$ and $f \mapsto \overline{S f}$ define a functor $\Sigma: \overline{\mathcal{M}} \rightarrow \overline{\mathcal{M}}$. Note that the construction of $\Sigma$ involves choices of both $I(M)$ and $I(f)$.

The proof of the following result is straightforward, $c f$. [25, Prop. 2.2] and the remark following. We leave the details to the reader.

Proposition 5.8. Let $(\mathcal{M}, X)$ be a Frobenius n-exact category. Then $\Sigma: \underline{\mathcal{M}} \mapsto \underline{\mathcal{M}}$ is an autoequivalence. Moreover, any two choices of assignments $M \mapsto \overline{S M}$ and $M \mapsto S^{\prime} M$ yield isomorphic functors.

Remark 5.9. Let $(\mathcal{M}, \mathcal{X})$ be a Frobenius $n$-exact category. In analogy with 39 , Sec. 2 ] we assume that $\Sigma: \underline{\mathcal{M}} \rightarrow \underline{\mathcal{M}}$ is not only an autoequivalence but an automorphism of $\underline{\mathcal{N}}$ (see also [21, Rmk. 2.2(d)]).

Let $(\mathcal{M}, X)$ be a Frobenius $n$-exact category. We define a class $\mathcal{S}=\mathcal{S}(X)$ of $n$ $\Sigma$-sequences in $\mathcal{M}$ as follows. Let $\alpha^{0}: X^{0} \rightarrow X^{1}$ be a morphism in $\mathcal{M}$. Then, for every morphism of $n$-exact sequences of the form

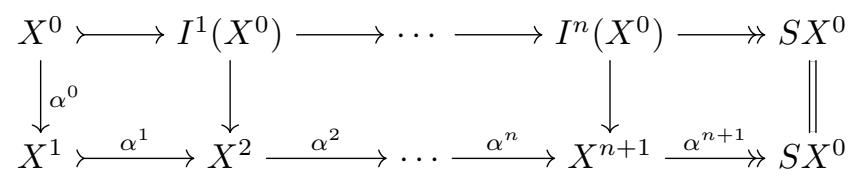

the sequence

$$
X^{0} \stackrel{\overline{\alpha^{0}}}{\longrightarrow} X^{1} \stackrel{\overline{\alpha^{1}}}{\longrightarrow} X^{2} \stackrel{\overline{\alpha^{2}}}{\longrightarrow} \cdots \stackrel{\overline{\alpha^{n}}}{\longrightarrow} X^{n+1} \stackrel{\overline{\alpha^{n+1}}}{\longrightarrow} \Sigma X^{0}
$$

is called a standard $(n+2)$-angle. An $n$ - $\Sigma$-sequence $Y$ in $\underline{\mathcal{M}}$ belongs to $\mathcal{S}$ if and only if it is isomorphic to a standard $(n+2)$-angle. 
We need the following result, which shows how admissible $n$-exact sequences give rise to standard $(n+2)$-angles, $c f$. [25, Lemma I.2.7].

Lemma 5.10. Let $(\mathcal{M}, X)$ be a Frobenius $n$-exact category and $X$ an admissible $n$-exact sequence in $\mathcal{M}$. The following statements hold:

(i) There exists a commutative diagram



(ii) The sequence

$$
X^{0} \stackrel{\overline{d^{0}}}{\longrightarrow} X^{1} \stackrel{\overline{d^{1}}}{\longrightarrow} \cdots \stackrel{\overline{d^{n}}}{\longrightarrow} X^{n+1} \stackrel{(-1)^{n} \overline{f^{n+1}}}{\longrightarrow} \Sigma X^{0}
$$

is a standard $(n+2)$-angle.

Proof. (i) The existence of the required commutative diagram follows from the fact that $I^{1}(M)$ is $X$-injective and $X$ is an $n$-exact sequence.

(ii) The dual of Proposition 4.8 implies that the mapping cone $C(f)$ of the right most part of 5.2 is an admissible $n$-exact sequence. For each $k \in\{1, \ldots, n\}$ we define

$$
g^{k}:=\left[\begin{array}{ll}
0 & (-1)^{k-1}
\end{array}\right]^{\top}: I^{k}\left(X^{0}\right) \longrightarrow X^{k+1} \oplus I^{k}\left(X^{0}\right) .
$$

It readily follows that the diagram

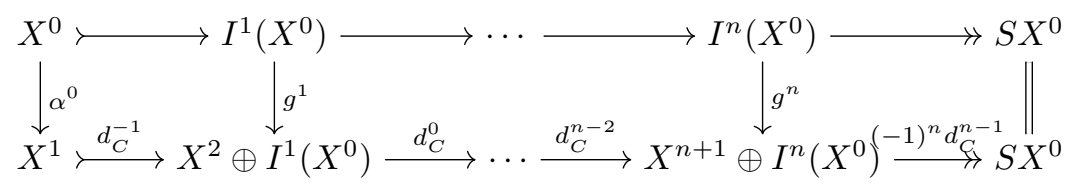

is commutative, and that it gives rise to the standard $(n+2)$-angle

$$
X^{0} \stackrel{\overline{d^{0}}}{\longrightarrow} X^{1} \stackrel{\overline{d^{1}}}{\longrightarrow} \cdots \stackrel{\overline{d^{n}}}{\longrightarrow} X^{n+1} \stackrel{(-1)^{n} \overline{f^{n+1}}}{\longrightarrow} \Sigma X^{0} .
$$

The following result is a higher analog of [25, Thm. I.2.6].

Theorem 5.11. Let $(\mathcal{M}, X)$ a Frobenius n-exact category. Then, $(\underline{\mathcal{N}}, \Sigma, \mathcal{S}(X))$ is an $(n+2)$-angulated category.

Proof. We need to show that $(\underline{\mathcal{M}}, \Sigma, \mathcal{S}(\mathcal{X}))$ satisfies the axioms of $(n+2)$-angulated categories, see Definition 5.1. For axioms (F1), (F2) and (F3) our proof is an adaptation of the proof of [25, Thm. I.2.6].

(F1) Firstly, recall that $X$ is closed under direct sums and direct summands, see Propositions 4.6 and 4.12 . It is easy to show that this implies that the same is true for $\mathcal{S}$. Secondly, the diagram

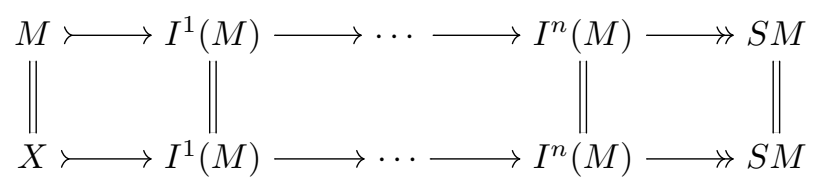


shows that the $n$ - $\Sigma$-sequence

$$
X \stackrel{1}{\longrightarrow} X \longrightarrow 0 \longrightarrow 0 \longrightarrow x X
$$

is a standard $(n+2)$-angle. Finally, by the definition of the class $\mathcal{S}$, every morphism is the first morphism of some standard $(n+2)$-angle. This shows that $(\underline{\mathcal{M}}, \Sigma, \mathcal{S}(\mathcal{X}))$ satisfies axiom (F1).

(F2) It suffices to consider the case of standard $(n+2)$-angles. Let



be a commutative diagram giving rise to the standard $(n+2)$-angle

$$
X^{0} \stackrel{\overline{\alpha^{0}}}{\longrightarrow} X^{1} \stackrel{\overline{\alpha^{1}}}{\longrightarrow} \cdots \stackrel{\overline{\alpha^{n}}}{\longrightarrow} X^{n+1} \stackrel{\overline{\alpha^{n+1}}}{\longrightarrow} \Sigma X^{0} .
$$

We need to show that its left rotation is a standard $(n+2)$-angle.

Firstly, by the definition of $\Sigma: \underline{\mathcal{N}} \rightarrow \underline{\mathcal{M}}$ we have a commutative diagram

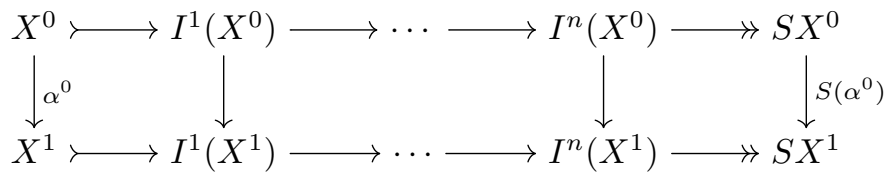

Secondly, Proposition 4.9 yields a commutative diagram

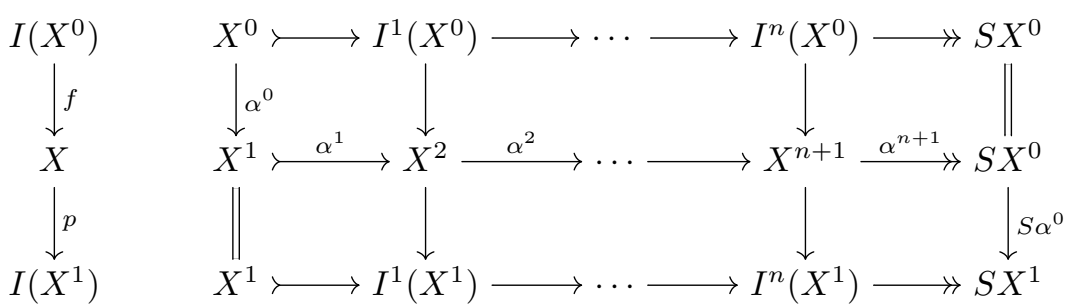

By the dual of Proposition 4.8, the mapping cone $C=C(p)$ is an admissible exact sequence. Thirdly, for each $k \in\{1, \ldots, n\}$ we define

$$
g^{k}:=\left[\begin{array}{ll}
0 & (-1)^{k-1}
\end{array}\right]^{\top}: I^{k}\left(X^{1}\right) \longrightarrow X^{k+2} \oplus I^{k}\left(X^{1}\right)
$$

(by convention, $X^{n+1}:=\Sigma X^{0}$ ). It follows that the diagram

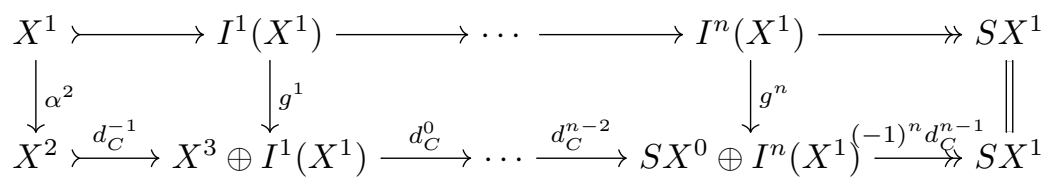

is commutative. Note that the bottom row is an admissible $n$-exact sequence for it is isomorphic to $C$. Finally, the standard $(n+2)$-angle induced by this diagram is isomorphic in $\underline{\mathcal{M}}$ to the left rotation of 5.4 . 
Conversely, suppose that there is a commutative diagram

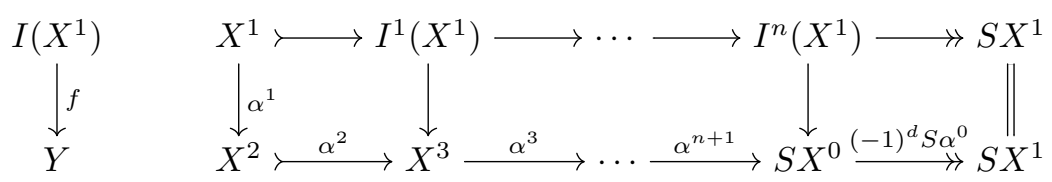

which gives rise to a standard $(n+2)$-angle of the form

$$
X^{1} \stackrel{\overline{\alpha^{1}}}{\longrightarrow} X^{2} \stackrel{\overline{\alpha^{2}}}{\longrightarrow} \cdots \stackrel{\overline{\alpha^{n}}}{\longrightarrow} X^{n+1} \stackrel{\overline{\alpha^{n+1}}}{\longrightarrow} \Sigma X^{0} \stackrel{(-1)^{n} \Sigma \overline{\alpha^{0}}}{\longrightarrow} \Sigma X^{1}
$$

On one hand, the definition of $\Sigma: \underline{\mathcal{M}} \rightarrow \underline{\mathcal{M}}$ yields the top two rows in the following commutative diagram:

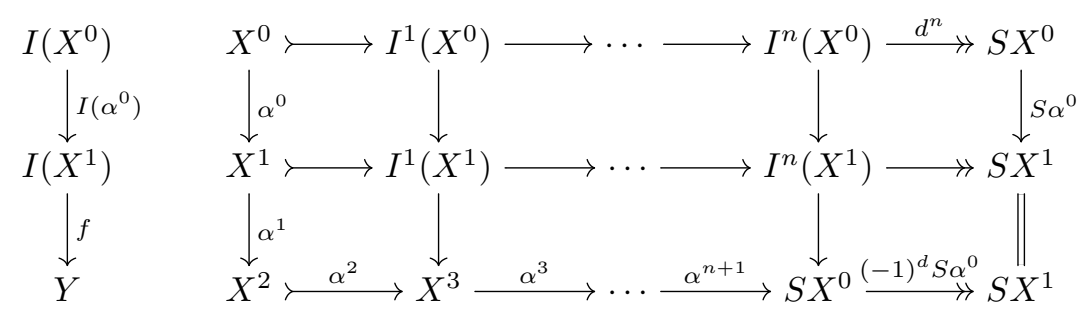

On the other hand, we have a commutative diagram

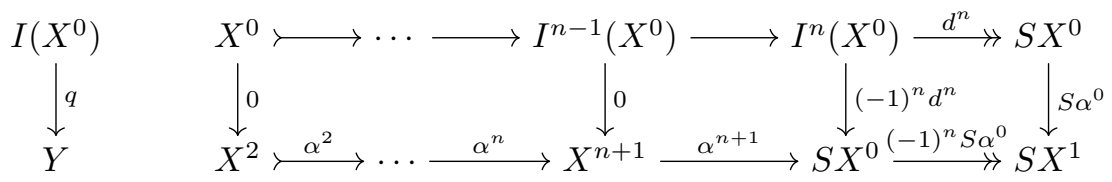

Then, the dual of the Comparison Lemma 2.1 implies the existence of a homotopy $h: I\left(\alpha^{0}\right) f \rightarrow q$. For each $k \in\{1, \ldots, n\}$ we define

$$
g^{k}:=\left[\left[\begin{array}{ll}
\left.(-1)^{k} I^{k}\left(\alpha^{0}\right) \quad(-1)^{k-1} h^{k}\right]
\end{array}\right]^{\top}: I^{k}\left(X^{0}\right) \longrightarrow I^{k}\left(X^{k}\right) \oplus X^{k+1} .\right.
$$

It is straightforward to verify that the diagram



commutes, where the bottom row is given by $C(f)$. Finally, the standard $(n+2)$ angle induced by this diagram is isomorphic to the $n$ - $\Sigma$-sequence

$$
X^{0} \stackrel{\overline{\alpha^{0}}}{\longrightarrow} X^{1} \stackrel{\overline{\alpha^{1}}}{\longrightarrow} \cdots \stackrel{\overline{\alpha^{n}}}{\longrightarrow} X^{n+1} \stackrel{\overline{\alpha^{n+1}}}{\longrightarrow} \Sigma X^{0} .
$$

This shows that $(\underline{\mathcal{X}}, \mathcal{X}, \mathcal{S}(\mathcal{X}))$ satisfies axiom (F2). 
(F3) for standard $(n+2)$-angles. Let

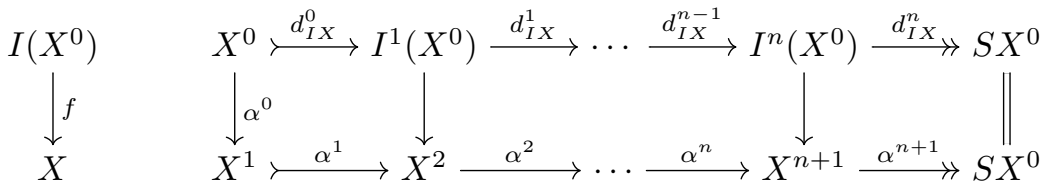

and



be pushout diagrams in $\mathcal{M}$. We set $C:=C(f)$.

Also, let $\varphi^{0}: X^{0} \rightarrow Y^{0}$ and $\varphi^{1}: X^{1} \rightarrow Y^{1}$ be morphisms such that $\overline{\varphi^{0} \beta^{0}}=\overline{\alpha^{0} \varphi^{1}}$. Thus, we have a diagram

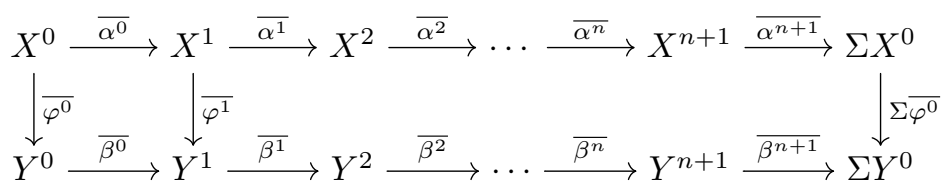

whose rows are standard $(n+2)$-angles. Recall that by the definition of $\Sigma: \underline{\mathcal{M}} \rightarrow \underline{\mathcal{M}}$, there is a commutative diagram

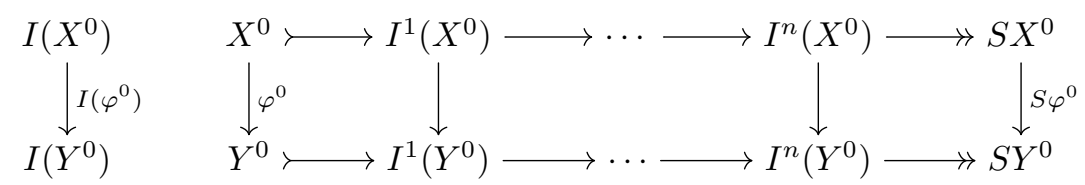

We shall construct a commutative diagram

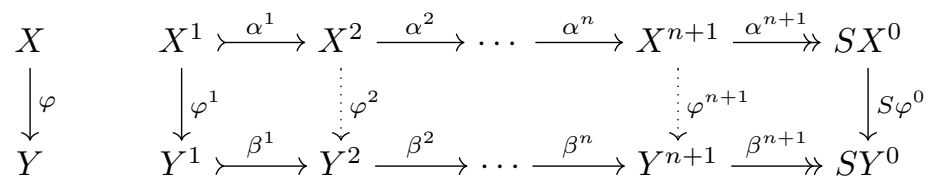

together with a homotopy $h: f \varphi \rightarrow I\left(\varphi^{0}\right) g$ such that $h^{n+1}: S X^{0} \rightarrow Y^{n+1}$ is the zero morphism. Note that this gives the required completion of diagram (5.5).

We begin with the construction of $h^{1}$ and $\varphi^{2}$. Since $\overline{\varphi^{0} \beta^{0}}=\overline{\alpha^{0} \varphi^{1}}$, there exists an $\mathcal{X}$-injective object $I \in \mathcal{M}$ and morphisms $u: X^{0} \rightarrow I$ and $v: I \rightarrow Y^{1}$ such that $\alpha^{0} \varphi^{1}-\varphi^{0} \beta^{0}=u v$. Then, given that $d_{I X}^{0}$ is an admissible monomorphism and $I$ is $X$-injective, we can construct a commutative diagram




Hence $\alpha^{0} \varphi^{1}-\varphi^{0} \beta^{0}=d_{I X}^{0} h^{1}$, as required. Then we have

$$
\begin{aligned}
d_{I X}^{0}\left(I\left(\varphi^{0}\right)^{1} g^{1}+h^{1} \beta^{1}\right) & =\varphi^{0} d_{I Y}^{0} g^{1}+\left(\alpha^{0} \varphi^{1}-\varphi^{0} g^{1}\right) \beta^{1} \\
& =\alpha^{0} \varphi^{1} \beta^{1} .
\end{aligned}
$$

Since $d_{C}^{0}$ is a weak cokernel of $d_{C}^{-1}$, there exists morphisms $\varphi^{2}: X^{2} \rightarrow Y^{2}$ and $h^{2}: I^{2}\left(X^{0}\right) \rightarrow Y^{2}$ such that $\varphi^{1} \beta^{1}=\alpha^{1} \varphi^{2}$ and $f^{1} \varphi^{2}-\left(I\left(\varphi^{0}\right)^{1} g^{1}+h^{1} \beta^{1}\right)=d_{I X}^{2} h^{2}$ or, equivalently,

$$
f^{1} \varphi^{2}-I\left(\varphi^{0}\right)^{1} g^{1}=h^{1} \beta^{1}+d_{I X}^{1} h^{2}
$$

Let $2 \leq k \leq n$ and suppose that for each $\ell \leq k$ we have constructed morphisms $\varphi^{\ell}: X^{\ell} \rightarrow Y^{\ell}$ and $h^{\ell}: I^{\ell}\left(X^{0}\right) \rightarrow Y^{\ell}$ such that $\alpha^{\ell-1} \varphi^{\ell}=\varphi^{\ell-1} \beta^{\ell-1}$ and

$$
f^{\ell-1} \varphi^{\ell}-I\left(\varphi^{0}\right)^{\ell-1} g^{\ell-1}=h^{\ell-1} \beta^{\ell-1}+d_{I X}^{\ell-1} h^{\ell} .
$$

Then we have

$$
\begin{aligned}
d_{I X}^{k-1}\left(I\left(\varphi^{0}\right)^{k} g^{k}+h^{k} \beta^{k}\right) & =I\left(\varphi^{0}\right)^{k-1} d_{I Y}^{k-1} g^{k}+\left(f^{k-1} \varphi^{k}-I\left(\varphi^{0}\right)^{k-1} g^{k-1}-h^{k-1} \beta^{k-1}\right) \beta^{k} \\
& =f^{k-1} \varphi^{k} \beta^{k} .
\end{aligned}
$$

Moreover, $\alpha^{k-1}\left(\varphi^{k} \beta^{k}\right)=$ Since $d_{C}^{k-1}$ is a weak cokernel of $d_{C}^{k-2}$, there exists morphisms $\varphi^{k+1}: X^{k+1} \rightarrow Y^{k+1}$ and $h^{k+1}: I^{k+1}\left(X^{0}\right) \rightarrow Y^{k+1}$ such that $\alpha^{k} \varphi^{k+1}=$ $\varphi^{k} \beta^{k}$ and

$$
f^{k} \varphi^{k+1}-I\left(\varphi^{0}\right)^{k} g^{k}=h^{k} \beta^{k}+d_{I X}^{k} h^{k+1} .
$$

This finishes the induction step.

It remains to show that $\alpha^{n+1} S \varphi^{0}=\varphi^{n+1} \beta^{n+1}$. Indeed, we have $\alpha^{n}\left(\alpha^{n+1} S \varphi^{0}\right)=$ 0 and $\alpha^{n}\left(\varphi^{n+1} \beta^{n+1}\right)=\varphi^{n}\left(\beta^{n} \beta^{n+1}\right)=0$. Moreover,

$$
f^{n+1}\left(\varphi^{n+1} \beta^{n+1}\right)=\left(I^{n}\left(\varphi^{0}\right) g^{n}-h^{n} \beta^{n}\right) \beta^{n+1}=d_{I X}^{n} S\left(\varphi^{0}\right)=f^{n+1}\left(\alpha^{n+1} S \varphi^{0}\right) .
$$

Since $d_{C}^{n-1}$ is a cokernel of $d_{C}^{n-2}$, we have $\alpha^{n+1} S\left(\varphi^{0}\right)=\varphi^{n+1} \beta^{n+1}$, as required.

This shows that $(\underline{\mathcal{X}}, \Sigma, \mathcal{S}(\mathcal{X}))$ satisfies axiom (F3) in the case of standard $(n+2)$ angles. The general case is left to the reader.

(F4) for standard $(n+2)$-angles. We shall show that the mapping cone of the morphism of standard $(n+2)$-angles that we constructed in the proof of axiom (F3) is a $(n+2)$-angle. We keep the notation and morphisms of the previous paragraphs.

For each $k \in\{0,1 \ldots, n-1\}$ we define

$$
r^{k}:=\left[\varphi^{k+1} g^{k}\right]: X^{k+1} \oplus I^{k}\left(Y^{0}\right) \longrightarrow Y^{k+1} .
$$

Also, we define

and

$$
r^{n}:=\left[\begin{array}{cc}
\alpha^{n+1} & 0 \\
\varphi^{n+1} & g^{n}
\end{array}\right]: X^{n+1} \longrightarrow I^{n}\left(Y^{0}\right) \rightarrow Y^{n+1}
$$

$$
r^{n+1}:=\left[\begin{array}{cc}
1_{S X^{0}} & 0 \\
S \varphi^{0} & 1_{S Y^{0}}
\end{array}\right]: S X^{0} \oplus S Y^{0} \longrightarrow S X^{0} \oplus S Y^{0} .
$$

It is straightforward to check that this defines a morphism of admissible $n$-exact sequences

$$
r: X \oplus I\left(Y^{0}\right) \longrightarrow T\left(S X^{0}, 0\right) \oplus Y .
$$

(recall that the direct sum of two admissible $n$-exact sequences is again an admissible $n$-exact sequence, see Proposition 4.6). Since $r^{n+1}$ is an isomorphism, we have that the mapping cone $C(r)$ is an admissible $n$-exact sequence, see Proposition 4.8 
Next, by the definition of $\Sigma: \underline{\mathcal{M}} \rightarrow \underline{\mathcal{M}}$ there is a commutative diagram

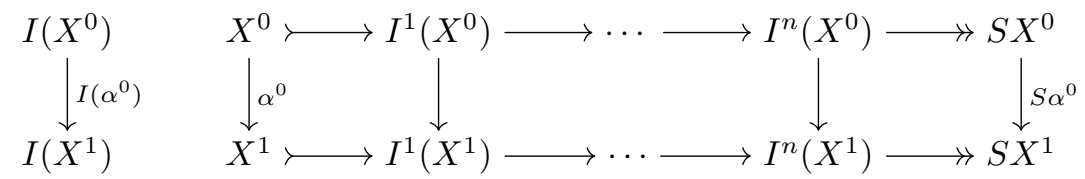

Then, by Proposition 4.9 and the dual of Proposition 4.8 there exists a commutative diagram



Let $t:=1_{X^{1}} \oplus 1_{Y^{0}}$. For each $k \in\{1, \ldots, n\}$ we define

and

$$
t^{k}:=\left[\begin{array}{ccc}
s^{k} & 0 & 0 \\
0 & 1_{I^{k}\left(Y^{0}\right)} & 0
\end{array}\right]: X^{k+1} \oplus I^{k}\left(Y^{0}\right) \oplus Y^{k} \longrightarrow I^{k}\left(X^{1}\right) \oplus I^{1}\left(Y^{0}\right)
$$

$$
t^{n+1}:=(-1)^{n}\left[\begin{array}{cc}
S \alpha^{0} & 0 \\
-S \varphi^{0} & \beta^{n+1}
\end{array}\right]: S X^{0} \oplus Y^{n+1} \longrightarrow S X^{1} \oplus S Y^{0} .
$$

It is readily verified that these morphisms define a morphism of admissible $n$-exact sequences

$$
t: C(r) \longrightarrow I\left(X^{1}\right) \oplus I\left(Y^{0}\right) .
$$

Finally, applying Lemma 5.10 to the morphism $t$ yields that the sequence

$$
X^{1} \oplus Y^{0} \stackrel{\gamma^{0}}{\longrightarrow} X^{2} \oplus Y^{1} \stackrel{\gamma^{1}}{\longrightarrow} \cdots \stackrel{\gamma^{n}}{\longrightarrow} \Sigma X^{0} \oplus Y^{n+1} \stackrel{\gamma^{n+1}}{\longrightarrow} \Sigma X^{1} \oplus \Sigma Y^{0}
$$

where for each $k \in\{0, \ldots, n\}$ we have

is a standard $(n+2)$-angle.

$$
\gamma^{k}=\left[\begin{array}{cc}
-\bar{\alpha}^{k+1} & 0 \\
\bar{\varphi}^{k+1} & \bar{\beta}^{k}
\end{array}\right]: X^{k+1} \oplus Y^{k} \longrightarrow X^{k+2} \oplus Y^{k+1}
$$

This shows that $(\underline{\mathcal{M}}, \Sigma, \mathcal{S}(\mathcal{X}))$ satisfies axiom (F4) in the case of standard $(n+2)$ angles. The general case is left to the reader.

Theorem 5.11 allows us to define the following class of $(n+2)$-angulated categories.

Definition 5.12. We say that a $(n+2)$-angulated category $\left(\mathcal{F}, \Sigma_{\mathcal{F}}, \mathcal{S}\right)$ is algebraic if there exists a Frobenius $n$-exact category $(\mathcal{N}, X)$ together with an equivalence of $(n+2)$-angulated categories between $\left(\underline{\mathcal{M}}, \Sigma_{\mathcal{M}}, \mathcal{S}(\mathcal{X})\right)$ and $\left(\mathcal{F}, \Sigma_{\mathcal{F}}, \mathcal{S}\right)$.

5.3. Standard construction. We remind the reader of the definition of an $n$ cluster-tilting subcategory of a triangulated category.

Definition 5.13. Let $(\mathcal{T}, \Sigma, \mathcal{S})$ be a triangulated category and $\mathcal{M}$ a subcategory of $\mathcal{T}$. We say that $\mathcal{M}$ is an $n$-cluster-tilting subcategory of $\mathcal{T}$ if $\mathcal{M}$ is functorially finite (see subsection 2.1) in $\mathcal{T}$ and

$$
\begin{aligned}
\mathcal{M} & =\left\{X \in \mathcal{T} \mid \forall i \in\{1, \ldots, n-1\} \operatorname{Ext}_{\mathcal{T}}^{i}(X, \mathcal{M})=0\right\} \\
& =\left\{X \in \mathcal{T} \mid \forall i \in\{1, \ldots, n-1\} \quad \operatorname{Ext}_{\mathcal{T}}^{i}(\mathcal{M}, X)=0\right\} .
\end{aligned}
$$


In [21, Sec. 4], Geiß-Keller-Oppermann give a standard construction of $(n+2)$ angulated categories from $n$-cluster-tilting categories of a triangulated category which are closed under the $n$-th power of the suspension functor. More precisely, they prove the following theorem.

Theorem 5.14. 21, Thm. 1] Let $\left(\mathcal{T}, \Sigma_{3}, \mathcal{S}\right)$ be a triangulated category with an $n$-cluster-tilting subcategory $\mathcal{C}$ such that $\Sigma_{3}^{n}(\mathcal{C}) \subseteq \mathcal{C}$. Then, $\left(\mathcal{C}, \Sigma_{3}^{n}, \mathcal{S}(\mathcal{C})\right)$ is an $(n+2)$-angulated category where $\mathcal{S}(\mathcal{C})$ is the class of all sequences

$$
X^{0} \stackrel{\alpha^{0}}{\longrightarrow} X^{1} \stackrel{\alpha^{1}}{\longrightarrow} X^{2} \stackrel{\alpha^{2}}{\longrightarrow} \cdots \stackrel{\alpha^{n}}{\longrightarrow} X^{n+1} \stackrel{\alpha^{n+1}}{\longrightarrow} \Sigma X^{0} .
$$

such that there exists a diagram

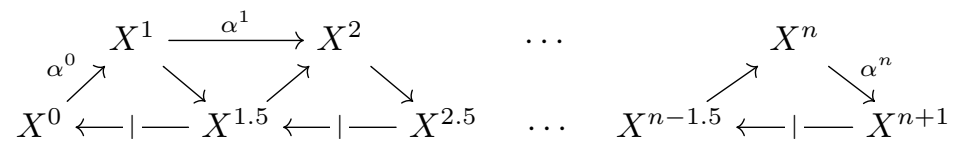

with $X^{k} \in \mathcal{C}$ for all $k \in \mathbb{Z}$ such that all oriented triangles are triangles in $\mathcal{T}$, all non-oriented triangles commute, and $\alpha^{n+1}$ is the composition along the lower edge of the diagram.

Our aim is to give an analogous construction for Frobenius $n$-exact categories. For this, we need some terminology.

Let $(\mathcal{E}, X)$ be a Frobenius exact category and $E \in \mathcal{E}$. An $n$-th cosyzygy $\mho^{n}(E)$ of $E$ is defined by an acyclic complex

$$
E \succ I^{1} \longrightarrow \cdots \longrightarrow I^{n} \longrightarrow \mho^{n}(E)
$$

where for all $k \in\{1, \ldots, n\}$ the object $I^{k}$ is $X$-injective.

Proposition 5.15. Let $(\mathcal{E}, X)$ be an exact category, $\mathcal{M}$ an n-cluster-tilting subcategory of $\mathcal{E}$ and $M \in \mathcal{M}$. If an $n$-th cosyzygy $\mho^{n}(M)$ of $M$ satisfies $\mho^{n}(M) \in \mathcal{M}$, then so does any other $n$-th cosyzygy $\tilde{\mho}^{n}(M)$ of $M$.

Proof. Note that for all $k \in\{1, \ldots, n-1\}$ we have

$$
\operatorname{Ext}_{x}^{k}\left(-, \mho^{n}(E)\right) \cong \operatorname{Ext}_{x}^{k+n}(-, E) \cong \operatorname{Ext}_{x}^{k}\left(-, \tilde{\mho}^{n}(E)\right) \text {. }
$$

Then, it follows from the definition of $n$-cluster-tilting subcategory that $\mho^{n}(E) \in \mathcal{M}$ if and only if $\tilde{\mho}^{n}(E) \in \mathcal{M}$.

Let $(\mathcal{E}, X)$ be a Frobenius exact category and for each $E \in \mathcal{E}$ fix a choice of $n$-th cosyzygy $\mho^{n}(E)$ of $E$. This defines a map on objects $\mho^{n}: \operatorname{Obj}(\mathcal{E}) \rightarrow \operatorname{Obj}(\mathcal{E})$. Note that if $\mathcal{M}$ is an $n$-cluster-tilting subcategory of $\mathcal{E}$, then Proposition 5.15 shows that the condition $\mho^{n}(\mathcal{M}) \subseteq \mathcal{M}$ is independent of the choice of $\mho^{n}$. We have the following result, which is closely related to Theorem 5.14 .

Theorem 5.16. Let $(\mathcal{E}, X)$ be a Frobenius exact category with an n-cluster-tilting subcategory $\mathcal{M}$ such that $\mho^{n}(\mathcal{M}) \subseteq \mathcal{M}$, and let $(\mathcal{M}, y)$ be the $n$-exact structure on $\mathcal{M}$ given in Theorem 4.14. Then, the following statements hold:

(i) The pair $(\mathcal{N}, y)$ is a Frobenius n-exact category.

(ii) Let $\left(\underline{\mathcal{E}}, \Sigma_{\underline{\varepsilon}}, \mathcal{S}(X)\right)$ be the standard triangulated structure of $\underline{\mathcal{E}}$. Then, $\underline{\mathcal{N}}$ is an $n$-cluster-tilting subcategory of $\underline{\mathcal{E}}$. 
(iii) Let $(\underline{\mathcal{M}}, \Sigma, \mathcal{S}(\underline{\mathcal{M}}))$ be the standard $(n+2)$-angulated structure of $\underline{\mathcal{M}}$. Then, we have an equivalence of $(n+2)$-angulated categories between $(\underline{\mathcal{M}}, \Sigma, \mathcal{S}(\underline{\mathcal{M}}))$ and $\left(\underline{\mathcal{M}}, \Sigma_{\underline{\mathcal{E}}}^{n}, \mathcal{S}(\underline{\mathcal{M}})\right)$.

Proof. (i) By Theorem 4.14 we have that $(\mathcal{M}, y)$ is an $n$-exact category; thus we only need to show that it is Frobenius. Indeed, note that the definition of $n$ cluster-tilting subcategory implies that $\mathcal{X}$ contains all $\mathcal{X}$-injective objects. Moreover, since $\mathcal{X}$-admissible monomorphisms with terms in $\mathcal{M}$ are precisely the $y$ admissible monomorphisms, all $\mathcal{X}$-injectives are also $\boldsymbol{y}$-injectives. Finally, the condition $\mho(\mathcal{M}) \subseteq \mathcal{M}$ implies that $(\mathcal{M}, y)$ has enough $y$-injectives. By duality, $(\mathcal{M}, y)$ has enough $\mathcal{Y}$-projectives (and they are the $\mathcal{X}$-projectives). Since $\mathcal{X}$-projectives and $\mathcal{X}$-projectives coincide, this shows that $(\mathcal{M}, \mathcal{y})$ is a Frobenius $n$-exact category.

(ii) This statement follows readily from the definitions.

(iii) For simplicity, we assume that $\Sigma_{\underline{\mathcal{M}}}$ and $\Sigma_{\mathcal{\varepsilon}}^{n}$ are equal and not only isomorphic (note that $\Sigma_{\underline{\mathcal{M}}}$ is induced by the $n$-th power of the cosyzygy in $\mathcal{E}$ ). By Proposition 5.2 it is enough to show that $\mathcal{S}(\underline{\mathcal{M}}) \subseteq \mathcal{S}(y)$. For this, recall that a standard triangle $A \stackrel{\bar{u}}{\rightarrow} B \stackrel{\bar{v}}{\rightarrow} C \stackrel{\bar{w}}{\rightarrow} \Sigma_{\underline{\mathcal{\varepsilon}}} A$ in $\mathcal{S}(X)$ is given by a morphism of admissible $X$-exact sequences

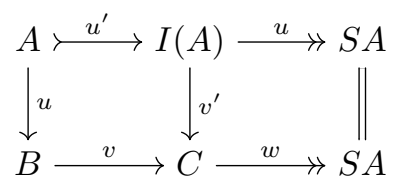

where $I(A)$ is an $\mathcal{X}$-injective object. By Proposition 4.8 this gives rise to an $X$ admissible exact sequence

$$
A \succ \stackrel{\left[\begin{array}{l}
u^{\prime} \\
u
\end{array}\right]}{\longrightarrow} I(A) \oplus B \stackrel{\left[v^{\prime}\right.}{\stackrel{v}{\longrightarrow}} \gg C .
$$

Consider a $(n+2)$-angle $X \in \mathcal{S}(\underline{\mathcal{M}})$

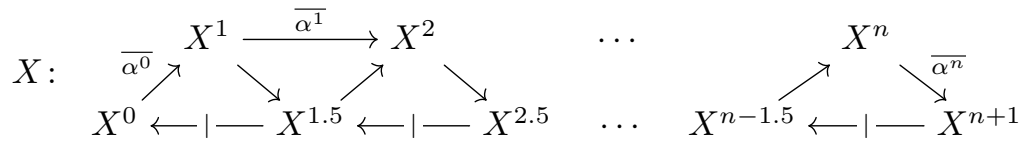

such that each of the involved triangles is a standard triangle in $(\underline{\mathcal{E}}, \mathcal{S}(\mathcal{X}))$ with $\overline{\alpha^{n+1}}$ the composition along the lower edge of the diagram. By gluing the associated $X$-admissible exact sequences associated to each of these triangles we obtain a $y$ admissible $n$-exact sequence

$$
X^{0} \succ I\left(X^{0}\right) \oplus X^{1} \longrightarrow \cdots \longrightarrow I\left(X^{n-1.5}\right) \oplus X^{n} \rightarrow X^{n+1} .
$$

Lemma 5.10 implies that this $n$-exact sequence induces a standard $(n+2)$-angle $X^{\prime} \in \mathcal{S}(y)$

$$
X^{\prime}: \quad X^{0} \stackrel{\overline{\alpha^{0}}}{\longrightarrow} X^{1} \stackrel{\overline{\alpha^{1}}}{\longrightarrow} \cdots \stackrel{\overline{\alpha^{n}}}{\longrightarrow} X^{n+1} \longrightarrow \Sigma_{\underline{\underline{\mathcal{M}}}} X^{0} .
$$

Finally, a straightforward verification shows that one can take $X^{n+1} \rightarrow \Sigma_{\underline{\mathcal{M}}} X^{0}$ in $X^{\prime}$ equal to $\overline{\alpha^{n+1}}$ showing that $X=X^{\prime}$ and the result follows. For example, for 
$n=2$ the last claim follows from Lemma 5.10 and the existence of a commutative diagram



The diagram needed for the general case can be easily inferred from the diagram above.

\section{EXAMPLES}

We conclude this article with a collection of examples of $n$-abelian, $n$-exact categories and algebraic $(n+2)$-angulated categories. Most of the examples we present are known, and all of them arise as $n$-cluster-tilting subcategories in different contexts. Our main tools in this section are Theorems 3.16, 4.14, and 5.16, In the remainder, $K$ denotes an algebraically closed field and all algebras are finite dimensional over $K$.

6.1. $n$-representation finite algebras. The class of $n$-representation finite algebras was introduced by Iyama-Oppermann in [32] in the context of higher AuslanderReiten theory as higher analogs of representation-finite algebras.

Definition 6.1. [32, Def. 2.2] Let $\Lambda$ be a finite dimensional algebra over a field $K$.

(i) A $\Lambda$-module $M \in \bmod \Lambda$ is an $n$-cluster-tilting module if add $M$ is an $n$ cluster-tilting subcategory of $\bmod \Lambda$. Note that Theorem 3.16 implies that add $M$ is an $n$-abelian category.

(ii) We say that $\Lambda$ is $n$-representation-finite if $\mathrm{gl} . \operatorname{dim} . \Lambda=n$ and there exists an $n$-cluster-tilting $\Lambda$-module.

The following result, observed jointly with Martin Herschend, gives examples of $n$-abelian categories for every positive integer $n$.

Proposition 6.2. Let $n \geq 1$ and $m \geq 0$. Also, let $\vec{A}_{n m+1}$ be the linearly oriented quiver of Dynkin type $A$ with $n m+1$ vertices, $J$ be the Jacobson radical of the path algebra $K \vec{A}_{n m+1}$, and $\Lambda:=K \vec{A}_{n m+1} / J^{2}$. Then, the following statements hold:

(i) There exists a unique basic $n$-cluster-tilting $\Lambda$-module $M$.

(ii) The category add $M \subseteq \bmod \Lambda$ is n-abelian.

Proof. We assume that $\vec{A}_{n m+1}$ has vertices $0,1, \ldots, n m$. Let $S_{i}$ be the simple module concentrated at the vertex $i$, and $P_{i}$ the indecomposable projective $\Lambda$ module with top $S_{i}$. The Auslander-Reiten quiver of $\bmod \Lambda$ is given by 


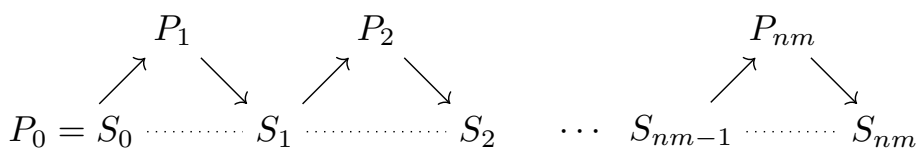

It is straightforward to verify that the module

$$
M:=\Lambda \oplus S_{n} \oplus S_{2 n} \oplus \cdots \oplus S_{(m-1) n} \oplus S_{n m}
$$

is the unique basic $n$-cluster-tilting $\Lambda$-module.

Let $\Lambda$ be a representation-finite algebra, i.e. such that the set of isomorphism classes of indecomposable $\Lambda$-modules is finite. We remind the reader that the Auslander algebra associated to $\Lambda$ is the endomorphism algebra of a basic $\Lambda$-module $M$ such that add $M=\bmod \Lambda$.

Example 6.3. Typical examples of 2-representation-finite algebras, hence sources of 2-abelian categories, are the Auslander algebras associated to $K Q$ where $Q$ is a Dynkin quiver $\vec{A}_{m}$, see [26, Sec. 9.2].

6.2. $n$-representation infinite algebras. The class of $n$-representation-infinite algebras was introduced by Herschend-Iyama-Oppermann in [28] as a higher analog of representation-infinite hereditary algebras from the viewpoint of higher AuslanderReiten theory. This class of algebras complements that of $n$-representation-finite algebras.

Let $\Lambda$ be a finite dimensional algebra with finite global dimension. Then, $\mathrm{D}^{\mathrm{b}}(\bmod \Lambda)$, the bounded derived category of $\bmod \Lambda$, has a Serre functor

$$
\nu:=-\otimes_{\Lambda}^{L} D \Lambda: \mathrm{D}^{\mathrm{b}}(\bmod \Lambda) \longrightarrow \mathrm{D}^{\mathrm{b}}(\bmod \Lambda) .
$$

We define $\nu_{n}:=\nu[-n]$.

Definition 6.4. 28, Def. 2.7] Let $\Lambda$ be a finite dimensional algebra such that gl. $\operatorname{dim} . \Lambda=n$. We say that $\Lambda$ is $n$-representation-infinite if for all $i \geq 0$ we have $\nu_{n}^{-i}(\Lambda) \in \bmod \Lambda$.

Let $\mathcal{T}$ be a triangulated category. Following [11, a $t$-structure on $\mathcal{T}$ is a pair $(\mathcal{T} \leq 0, \mathcal{T} \geq 0$ ) of strictly full (i.e. full and closed under isomorphisms) subcategories of $\mathcal{T}$ which satisfies the following properties:

(i) We have $\Sigma \mathcal{T} \leq 0 \subseteq \mathcal{T} \leq 0$ and $\Sigma^{-1} \mathcal{T} \geq 0 \subseteq \mathcal{T} \geq 0$.

(ii) For all $X \in \mathcal{T} \leq 0$ and for all $Y \in \mathcal{T} \geq 0$ we have $\mathcal{T}\left(X, \Sigma^{-1} Y\right)=0$.

(iii) For each $X \in \mathcal{T}$ there exists a triangle $X^{\prime} \rightarrow X \rightarrow X^{\prime \prime} \rightarrow \Sigma X^{\prime}$ with $X^{\prime} \in \mathcal{T} \leq 0$ and $X^{\prime \prime} \in \Sigma^{-1} \mathcal{T} \geq 0$.

The heart of $(\mathcal{T} \leq 0, \mathcal{T} \geq 0)$ is by definition $\mathcal{T} \leq 0 \cap \mathcal{T} \geq 0$. The heart of a $t$-structure is always an abelian category [11.

Note that $\mathrm{D}^{\mathrm{b}}(\bmod \Lambda)$ has a standard $t$-structure $\left(\mathcal{D}^{\leq 0}, \mathcal{D}^{\geq 0}\right)$ defined by

$$
\begin{aligned}
& \mathcal{D}^{\leq 0}:=\left\{X \in \mathrm{D}^{\mathrm{b}}(\bmod \Lambda) \mid \text { for all } i>0 H^{i}(X)=0\right\}, \\
& \mathcal{D}^{\geq 0}:=\left\{X \in \mathrm{D}^{\mathrm{b}}(\bmod \Lambda) \mid \text { for all } i<0 H^{i}(X)=0\right\} .
\end{aligned}
$$

The heart of $\left(\mathcal{D}^{\leq 0}, \mathcal{D}^{\geq 0}\right)$ is precisely $\bmod \Lambda$. 
Theorem 6.5. 40, Thm. 3.7] Let $\Lambda$ be an n-representation infinite algebra such that $\Pi_{n+1}(\Lambda)$ is noetherian. Let $\left(\mathcal{D}^{\leq 0}, \mathcal{D}^{\geq 0}\right)$ be the standard $t$-structure of $\mathrm{D}^{\mathrm{b}}(\bmod \Lambda)$ and define

$$
\begin{aligned}
x^{\leq 0} & :=\left\{X \in \mathrm{D}^{\mathrm{b}}(\bmod \Lambda) \mid \nu_{n}^{-i}(X) \in \mathcal{D}^{\leq 0} \forall i \gg 0\right\} \\
x^{\geq 0} & :=\left\{X \in \mathrm{D}^{\mathrm{b}}(\bmod \Lambda) \mid \nu_{n}^{-i}(X) \in \mathcal{D}^{\geq 0} \forall i \gg 0\right\} .
\end{aligned}
$$

Then, the pair $(X \leq 0, X \geq 0)$ is a $t$-structure in $\mathrm{D}^{\mathrm{b}}(\bmod \Lambda)$. Moreover, the heart of this $t$-structure is equivalent to the non-commutative projective scheme $\mathrm{qgr} \Pi_{n+1}(\Lambda)$, see [2] for the definition.

The following result gives examples of $n$-exact categories.

Theorem 6.6. 27, Thm. 2.11] Let $\Lambda$ be an n-representation infinite algebra such that $\Pi_{n+1}(\Lambda)$ is noetherian. Let $\left(X^{\leq 0}, X^{\geq 0}\right)$ be the $t$-structure defined in Theorem 6.5 and $\mathcal{H}$ be its heart. Then, the following statements hold:

(i) The category

$$
\mathcal{E}:=\left\{X \in \mathcal{H} \mid \nu_{n}^{i}(X) \in(\bmod \Lambda)[-n] \forall i \gg 0\right\} .
$$

is an extension closed subcategory of $\mathcal{H}$.

(ii) The category

$$
\mathcal{U}:=\operatorname{add}\left\{\nu_{n}^{-i}(\Lambda) \mid i \in \mathbb{Z}\right\}
$$

is an $n$-cluster-tilting subcategory of $\mathcal{E}$.

With the notation of Theorem 6.6 note that $\mathcal{E}$ is an exact category hence Theorem 4.14 implies that $\mathcal{U}$ is an $n$-exact category.

We now give a concrete example of an $n$-exact category constructed using Theorem 6.6.

Example 6.7. Let $\operatorname{coh} \mathbb{P}_{K}^{n}$ be the category of coherent sheaves over the projective $n$-space over $K$, and let $\Lambda$ be the endomorphism algebra of the tilting bundle $\mathcal{O} \oplus \mathcal{O}(1) \oplus \cdots \oplus \mathcal{O}(n)$, see [10]. It is known that $\Lambda$ is an $n$-representation-infinite algebra and that $\Pi_{n+1}(\Lambda)$ is noetherian, see [28, Ex. 2.15]. Moreover, there is an equivalence of triangulated categories

$$
\mathrm{D}^{\mathrm{b}}(\bmod \Lambda) \cong \mathrm{D}^{\mathrm{b}}\left(\operatorname{coh} \mathbb{P}_{K}^{n}\right) .
$$

This equivalence induces an equivalence of exact categories between the category $\mathcal{E}$ given in Theorem 6.6 and vect $\mathbb{P}_{K}^{n}$, the category of vector bundles over $\mathbb{P}_{K}^{n}$. Also, it induces an equivalence of additive categories

$$
\mathcal{U} \cong \operatorname{add}\{\mathcal{O}(i) \mid i \in \mathbb{Z}\} .
$$

Finally, Theorem 4.14 implies that $\mathcal{U}$ is an $n$-exact category with respect to the class of all exact sequences

$$
0 \longrightarrow X^{0} \longrightarrow X^{1} \longrightarrow \cdots \longrightarrow X^{n} \longrightarrow X^{n+1} \longrightarrow 0
$$

with all terms in $\mathcal{U}$. 
6.3. Relative $n$-cluster-tilting subcategories. Let $\Lambda$ be a finite-dimensional algebra and $T$ a $\Lambda$-module. It is easy to see that the perpendicular category

$$
T^{\perp>0}:=\left\{M \in \bmod \Lambda \mid \forall k>0, \operatorname{Ext}_{\Lambda}^{k}(T, M)=0\right\}
$$

is exact for it is an extension closed subcategory of $\bmod \Lambda$. We have the following result.

Proposition 6.8. [31, Cor. 1.15] Let $Q$ be a Dynkin quiver. Then, there exists a tilting KQ-module $T$ of projective dimension 1 such that $T^{\perp>0}$ contains a 2-clustertilting subcategory $\mathcal{M}$.

Remark 6.9. With the notation of Proposition 6.8, the category $\mathcal{M}$ is 2 -exact by Theorem 4.14

More generally, in [31, Cor. 1.16] for each $n$ an algebra $\Lambda$ of global dimension at most $n$ such that there exists a tilting $\Lambda$-module $T$ of finite projective dimension and $T^{\perp>0}$ has an $n$-cluster-titling subcategory was constructed.

6.4. Isolated singularities. Let $R$ be a commutative complete Gorenstein ring of dimension $n$ with residue field $K$. The category of Cohen-Macaulay $R$-modules is by definition

$$
\mathrm{CM} R:=\{M \in \bmod R \mid \operatorname{depth} M=n\} .
$$

Note that CM $R$ is a Frobenius exact category, see [17, Rmk. 4.8].

We remind the reader that $R$ is an isolated singularity if $R$ is not regular and for all non-maximal prime ideals $\mathfrak{p} \subset R$ we have that $R_{\mathfrak{p}}$ is a regular ring. In this case CM $R$ has almost-split sequences, see [8, page 200] and [8, 46, Thm. 3.2].

Theorem 6.10. [30, Thm. 2.5] and [34, Cor. 8.2] Let $K$ be an algebraically closed field of characteristic 0 and set $S:=K \llbracket x_{0}, x_{1}, \ldots, x_{n} \rrbracket$. Also, let $G$ be a finite subgroup of $\mathrm{SL}_{n+1}(K)$ such that no element $\sigma \neq 1$ of $G$ has eigenvalue 1. Then $G$ acts on $S$ in a natural way and we define

$$
S^{G}:=\{s \in S \mid \forall g \in G, g \cdot s=s\} .
$$

Then, the following statements hold:

(i) The ring $S^{G}$ is an isolated singularity.

(ii) We have $S \in \mathrm{CM} S^{G}$.

(iii) The category add $S$ is an n-cluster-tilting subcategory of $\mathrm{CM} S^{G}$.

With the notation of Theorem 6.10, note that Theorem 4.14 implies that add $S$ is an $n$-exact category with respect to the class of all exact sequences

$$
0 \longrightarrow X^{0} \longrightarrow X^{1} \longrightarrow \cdots \longrightarrow X^{n} \longrightarrow X^{n+1} \longrightarrow 0
$$

in $\bmod S^{G}$ with terms in add $S$.

6.5. Algebraic $(n+2)$-angulated categories. In this subsection we revisit the examples of [21, Sec. 6.3] from the viewpoint of algebraic $(n+2)$-angulated categories. We remind the reader that we say that an object $T$ in a triangulated category $\mathcal{C}$ is $n$-cluster-tilting if add $T$ is an $n$-cluster-tilting subcategory of $\mathcal{C}$.

Let $\mathcal{C}$ be a algebraic triangulated category. Hence, there exists a Frobenius exact category $(\mathcal{E}, X)$ such that $\underline{\mathcal{E}}$ is equivalent to $\mathcal{C}$ as triangulated categories. It is easy to see that each $n$-cluster-tilting subcategory of $\mathcal{C}$ lifts to an $n$-cluster-tilting 
subcategory of $\mathcal{E}$ by including all the $\mathcal{X}$-injective objects in $\mathcal{E}$. By Theorem 5.16 , every $(n+2)$-angulated category constructed using Theorem 5.14 from an algebraic triangulated category is in turn an algebraic $(n+2)$-angulated category. Known examples of algebraic $(n+2)$-angulated categories arising in this way are the following:

- Let $\mathcal{C}=\mathcal{C}_{Q}$ be the cluster category associated with an acyclic quiver $Q$, see [16] for details. It is known that $\mathcal{C}$ is an algebraic triangulated category 38. Moreover, a basic 2-cluster-tilting object $T \in \mathcal{C}$ satisfies $\Sigma^{2} T \cong T$ if and only if $\mathcal{C}(T, T)$ is a selfinjective algebra, see [33, Cor. 3.8]. All such algebras were classified by Ringel in [43. In particular, such algebras exist only if $Q$ is a Dynkin quiver of type $D$ (including $D_{3}=A_{3}$ ). Hence, if $T$ is a 2-cluster-tilting object in $\mathcal{C}$ such that $\Sigma^{2} T \cong T$, then add $T \subseteq \mathcal{C}$ is an algebraic 4-angulated category.

- Let $\mathcal{C}=\mathcal{C}_{\mathbb{X}}$ be the cluster category associated with a weighted projective line, see 9 for details. As in the previous case, a 2-cluster-tilting object $T \in \mathcal{C}$ satisfies $\Sigma^{2} T \cong T$ if and only if $\mathcal{C}(T, T)$ is a selfinjective algebra. All such algebras are classified in [35, Thm. 1.3]. Such algebras exist if and only if $\mathbb{X}$ has tubular weight type $(2,2,2,2),(2,4,4)$, or $(2,3,6)$. If $T$ is a 2-cluster-tilting object in $\mathcal{C}$ such that $\Sigma^{2} T \cong T$, then add $T \subseteq \mathcal{C}$ is an algebraic 4-angulated category.

- Let $\Lambda$ be the preprojective algebra of type $A_{n}$. Recall that $\bmod \Lambda$ is a Frobenius abelian category hence the stable category $\bmod \Lambda$ is triangulated. It is known that the standard 2-cluster-tilting $\Lambda$-module $T$ corresponding to the linear orientation of $A_{n}$ satisfies $\mho^{2}(T) \cong T$, see 22 . It follows that $\operatorname{add} T \subseteq \bmod \Lambda$ is a Frobenius 2-exact category and thus $\underline{\operatorname{add}} T \subseteq \underline{\bmod } \Lambda$ is an algebraic 4-angulated category.

- Let $\Lambda$ be an $n$-representation-finite algebra. Then, [33, Cor. 3.7] implies that the canonical $n$-cluster-tilting object $\pi \Lambda$ in the associated Amiot $n$ cluster category $\mathcal{C}$ is stable under the Serre functor $\Sigma^{n}$. It is known that $\mathcal{C}$ is an algebraic triangulated category, see [33, Thm. 4.15], hence add $\pi \Lambda \subseteq \mathcal{C}$ is an algebraic $(n+2)$-angulated category.

We refer the reader to [21, Sec. 6] for more details.

\section{REFERENCES}

[1] C. Amiot, O. Iyama, and I. Reiten. Stable categories of Cohen-Macaulay modules and cluster categories. arXiv:1104.3658, Apr. 2011.

[2] M. Artin and J. Zhang. Noncommutative projective schemes. Adv. Math., 109(2):228-287, Dec. 1994.

[3] M. Auslander. Coherent functors. In Proc. Conf. Categorical Algebra (La Jolla, Calif., 1965), pages 189-231. Springer, New York, 1966.

[4] M. Auslander. Representation dimension of Artin algebras. Lecture Notes. Queen Mary College, London, 1971.

[5] M. Auslander and I. Reiten. Stable equivalence of dualizing r-varieties. Adv. Math., 12(3):306366, Mar. 1974.

[6] M. Auslander and I. Reiten. Applications of contravariantly finite subcategories. Adv. Math., 86(1):111-152, Mar. 1991.

[7] M. Auslander and S. O. Smalø. Almost split sequences in subcategories. J. Algebra, 69(2):426454, Apr. 1981.

[8] M. Auslander and L. Unger. Isolated singularities and existence of almost split sequences. In V. Dlab, P. Gabriel, and G. Michler, editors, Representation Theory II Groups and Orders, 
number 1178 in Lecture Notes in Mathematics, pages 194-242. Springer Berlin Heidelberg, Jan. 1986.

[9] M. Barot, D. Kussin, and H. Lenzing. The cluster category of a canonical algebra. Trans. Amer. Math. Soc., 362(08):4313-4330, Mar. 2010.

[10] A. Beilinson. Coherent sheaves on $\mathrm{P}^{d}$ and problems in linear algebra. Funktsional. Anal. $i$ Prilozhen, 12(3):68-69, 1978.

[11] A. A. Beilinson, J. Bernstein, and P. Deligne. Faisceaux pervers. In Analysis and topology on singular spaces, I (Luminy, 1981), volume 100 of Astérisque, pages 5-171. Soc. Math. France, Paris, 1982.

[12] P. A. Bergh and M. Thaule. The axioms for $n$-angulated categories. Algebr. Geom. Topol., 13(4):2405-2428, July 2013.

[13] P. A. Bergh and M. Thaule. Higher $n$-angulations from local algebras. arXiv:1311.2089 [math], Nov. 2013.

[14] P. A. Bergh and M. Thaule. The Grothendieck group of an $n$-angulated category. J. Pure Appl. Algebra, 218(2):354-366, Feb. 2014.

[15] A. I. Bondal and M. M. Kapranov. Framed triangulated categories. Math. Sb., 181(5):669683, 1990.

[16] A. B. Buan, R. Marsh, M. Reineke, I. Reiten, and G. Todorov. Tilting theory and cluster combinatorics. Adv. Math., 204(2):572-618, Aug. 2006.

[17] R.-O. Buchweitz. Maximal Cohen-Macaulay Modules and Tate-Cohomology Over Gorenstein Rings. 1986.

[18] T. Bühler. Exact categories. Expo. Math., 28(1):1-69, 2010.

[19] S. Fomin and A. Zelevinsky. Cluster algebras i: Foundations. J. Amer. Math. Soc., 15(02):497-529, Dec. 2001

[20] L. Frerick and D. Sieg. Exact categories in functional analysis, 2010.

[21] C. Geiß, B. Keller, and S. Oppermann. n-angulated categories. J. Reine Angew. Math., 675:101-120, 2013.

[22] C. Geiß, B. Leclerc, and J. Schröer. Auslander algebras and initial seeds for cluster algebras. J. London Math. Soc., 75(3):718-740, June 2007.

[23] C. Geiß, B. Leclerc, and J. Schröer. Preprojective algebras and cluster algebras. In Trends in representation theory of algebras and related topics, EMS Ser. Congr. Rep., pages 253-283. Eur. Math. Soc., Zürich, 2008.

[24] A. Grothendieck. Sur quelques points d'algèbre homologique, i. Tohoku Math. J. (2), 9(2):119-221, 1957.

[25] D. Happel. Triangulated Categories in the Representation Theory of Finite Dimensional Algebras. Number 119 in London Mathematical Society Lecture Note Series. Cambridge University Press, Cambridge, 1988.

[26] M. Herschend and O. Iyama. Selfinjective quivers with potential and 2-representation-finite algebras. Compos. Math., 147(06):1885-1920, 2011.

[27] M. Herschend, O. Iyama, H. Minamoto, and S. Oppermann. Representation theory of GeigleLenzing complete intersections. arXiv:1409.0668, Sept. 2014.

[28] M. Herschend, O. Iyama, and S. Oppermann. n-representation infinite algebras. Adv. Math., 252:292-342, Feb. 2014.

[29] O. Iyama. Auslander correspondence. Adv. Math., 210(1):51-82, Mar. 2007.

[30] O. Iyama. Higher-dimensional Auslander-Reiten theory on maximal orthogonal subcategories. Adv. Math., 210(1):22-50, Mar. 2007.

[31] O. Iyama. Cluster tilting for higher auslander algebras. Adv. Math., 226(1):1-61, Jan. 2011.

[32] O. Iyama and S. Oppermann. $n$-representation-finite algebras and $n$-APR tilting. Trans. Amer. Math. Soc., 363(12):6575-6614, July 2011.

[33] O. Iyama and S. Oppermann. Stable categories of higher preprojective algebras. Adv. Math., 244:23-68, Sept. 2013.

[34] O. Iyama and Y. Yoshino. Mutation in triangulated categories and rigid Cohen-Macaulay modules. Invent. Math., 172(1):117-168, Apr. 2008.

[35] G. Jasso. $\tau^{2}$-stable tilting complexes over weighted projective lines. arXiv:1402.6036, Feb. 2014.

[36] B. Keller. Chain complexes and stable categories. Manuscripta Math., 67(1):379-417, Dec. 1990. 
[37] B. Keller. On differential graded categories. In International Congress of Mathematicians. Vol. II, pages 151-190. Eur. Math. Soc., Zürich, 2006.

[38] B. Keller and I. Reiten. Acyclic Calabi-Yau categories. Compos. Math., 144(05):1332-1348, 2008.

[39] B. Keller and D. Vossieck. Sous les catégories dérivées. C. R. Acad. Sci. Paris Sér. I Math., 305(6):22-228, 1987.

[40] H. Minamoto. Ampleness of two-sided tilting complexes. Int. Math. Res. Not., 2012(1):67101, Jan. 2012.

[41] A. Neeman. The derived category of an exact category. J. Algebra, 135(2):388-394, Dec. 1990.

[42] D. Quillen. Higher algebraic $k$-theory. i. In Algebraic K-theory, I: Higher K-theories (Proc. Conf., Battelle Memorial Inst., Seattle, Wash., 1972), number 341 in Lecture Notes in Math., pages 85-147. Springer, Berlin, 1973.

[43] C. M. Ringel. The self-injective cluster-tilted algebras. Arch. Math., 91(3):218-225, Sept. 2008.

[44] J.-L. Verdier. Des catégories dérivées des catégories abéliennes. Astérisque, (239):xii+253 pp., 1996. With a preface by Luc Illusie, Edited and with a note by Georges Maltsiniotis.

[45] C. A. Weibel. An introduction to homological algebra, volume 38 of Cambridge Studies in Advanced Mathematics. Cambridge University Press, Cambridge, 1994.

[46] Y. Yoshino. Cohen-Macaulay Modules over Cohen-Macaulay Rings. Cambridge University Press, Cambridge, 1990.

E-mail address: gjasso@math.uni-bonn.de

$U R L$ : https://gustavo.jasso.info

Graduate School of Mathematics, Nagoya University. Furo-cho, Chikusa-ku. 4648602 NAGOYA, JAPAN.

Current address: Mathematik Zentrum, Universität Bonn, Endenicher Allee 60, 53115 Bonn, Germany 